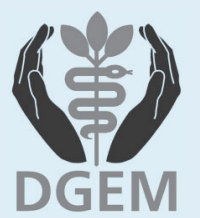

\title{
S3-Leitlinie der Deutschen Gesellschaft für Ernährungsmedizin (DGEM) in Zusammenarbeit mit der GESKES und der AKE Künstliche Ernährung im ambulanten Bereich
}

\author{
S3-Guideline of the German Society for Nutritional Medicine (DGEM) \\ in Cooperation with the GESKES and the AKE \\ Nutritional Support in the Homecare and Outpatient Sector
}

Autoren

Institute
S. C. Bischoff' , J. Arends² , F. Dörje ${ }^{3}$, P. Engeser ${ }^{4}$, G. Hanke ${ }^{5}$, K. Köchling ${ }^{1}$, A. H. Leischker ${ }^{6}$, S. Mühlebach7, A. Schneider ${ }^{8}$, C. Seipt ${ }^{8}$, D. Volkert ${ }^{9}$, U. Zech ${ }^{10}$, Z. Stanga ${ }^{11}$ und das DGEM Steering Committee*

Die Institute sind am Ende des Artikels gelistet.

Schlüsselwörter
Leitlinie
ambulanter Bereich
heimenterale Ernährung
heimparenterale Ernährung
orale Trinknahrung
Ernährungsteam
Keywords
guideline
sector
home enteral nutrition
home parenteral nutrition
nutrition support team

Bibliografie

DOI http://dx.doi.org/

10.1055/s-0033-1349549

Aktuel Ernahrungsmed 2013;

38: e101-e154

(c) Georg Thieme Verlag KG

Stuttgart · New York

ISSN 0341-0501

Korrespondenzadresse
Prof. Dr. med.
Stephan C. Bischoff
Universität Hohenheim,
Insitut für Ernährungsmedizin
Fruwirthstraße 12
70593 Stuttgart
Tel.: +49/711/459-24101
bischoff.stephan@uni-
hohenheim.de

\section{Zusammenfassung \\ $\nabla$}

Fragestellung: Künstliche Ernährung im ambulanten Bereich umfasst die heimparenterale Ernährung (HPE) und heimenterale Ernährung (HEE) sowie die supplementäre Trinknahrung (ONS), die bei Patienten zu Hause oder in Pflegeeinrichtungen durchgeführt wird. Meistens beginnen HPE/HEE im Anschluss an einen stationären Aufenthalt in einer Klinik. Die vorliegende Leitlinie soll evidenzbasierte Empfehlungen zur künstlichen Ernährung im ambulanten Bereich geben.

Methodik: Es wurde eine systematische Literaturrecherche zur HEE, HPE und zu ONS im ambulanten Bereich durchgeführt. Die Ergebnisse wurden in einer interdisziplinären Arbeitsgruppe aus Medizinern, Apothekern, Ernährungswissenschaftlern und Diätassistenten diskutiert. Auf dieser Basis wurden von der Arbeitsgruppe Empfehlungen erarbeitet, die auf der Konsensuskonferenz am 1. Dezember 2012 vorgestellt, diskutiert, zum Teil modifiziert und verabschiedet wurden.

Ergebnisse: Die Leitlinie enthält 54 Empfehlungen zur künstlichen Ernährung im ambulanten Bereich. Die Indikation zur künstlichen Ernährung im ambulanten Bereich unterscheidet sich nicht grundsätzlich von der Indikation im stationären Bereich, wobei die Aspekte Prognose, Lebensqualität und Ethik im ambulanten Bereich einen besonders hohen Stellenwert haben. Der geeignete Zufuhrweg für eine ambulante künstliche Ernährung hängt von der funktionellen Integrität des Magen-Darm-Trakts ab. Bei einer längerfristigen HEE soll eine perkutane endoskopische Gastrostomie (PEG)-Sonde gegenüber den herkömmlichen chirurgischen Gastrostomieverfahren aufgrund einer niedrigeren Komplikationsrate bevorzugt werden. Bei relevanten Störungen der

\footnotetext{
* DGEM Steering Committee: Bischoff SC, Lochs H, Weimann A, Adolph M, Ockenga J, Sieber C
}

\section{Abstract \\ $\nabla$}

Purpose: Nutritional support in the homecare and outpatient sector comprises home parenteral nutrition (HPN) and home enteral nutrition (HEN) as well as oral nutritional supplements (ONS), being administered in the patient's home or in nursing homes. In most cases the HPN/HEN starts following an in-patient treatment in hospital. The present guideline offers evidence-based recommendations for nutritional support in the outpatient or homecare sector.

Methods: A systematic literature search about HEN, HPN, and ONS in the homecare sector was conducted. The results were discussed in an interdisciplinary working group that consisted of physicians, pharmacists, nutrition scientists and dietitians. Based on this discussion and the literature, the working group developed recommendations, which were presented, discussed, partly modified and finally passed at the consensus conference on December 1st, 2012.

Results: The guideline includes 54 recommendations for nutritional support in the outpatient sector. The indication for nutritional support in the outpatient sector does not differ basically from the indication for the in-patient treatment. Criteria like prognosis, quality of life and ethical considerations play a more important role in the outpatient sector. The appropriate way of administration nutritional support in the homecare setting depends on the functional integrity of the gastrointestinal tract. In case of a long-term HEE a percutaneous endoscopic gastrostomy (PEG) tube should be preferred towards surgical gastrostomy procedures, because of lower complication rates. In case of relevant disorders of transport and absorptive capacity of the small intestine intravenous nutrition should be chosen. The nursing care of the tubes and catheters for HEN and HPN should be carried out by professional care attendants according to evidence-based guidlines and 
Transport- oder Absorptionskapazität des Dünndarms soll eine intravenöse Ernährung gewählt werden. Die Pflege der Zugangswege bei HEE und HPE sollte durch geschultes Pflegefachpersonal gemäß evidenzbasierten Richtlinien und Pflegestandards durchgeführt werden, um eine hohe Hygienequalität zu erreichen. Für die ausschließliche und langfristige HEE über gastrointestinale Sonden und für die orale Ernährung durch Trinknahrung sollen vollständig bilanzierte Trink- bzw. Sondennahrungsprodukte verwendet werden, da diese in ihrer Zusammensetzung den D-A-CH-Empfehlungen entsprechen und den EU-Richtlinien genügen. Zur HPE sollten „All-in-One-Nährmischungen“ bzw. „Dreikammerbeutel“ verwendet werden, denn sie sind als sichere, effektive und risikoreduzierende Standards zur Durchführung der HPE etabliert.

Schlussfolgerung: Die HEE und HPE sind anerkannte und sichere Verfahren, wenn sie standardisiert nach aktuellen wissenschaftlichen Erkenntnissen und koordiniert möglichst durch ein interdisziplinäres Ernährungsteam erfolgen. Durch das Ernährungsteam können die Qualität der Maßnahmen erhöht und die Komplikationsraten reduziert werden. Die künstliche Ernährung im ambulanten Bereich kann einen entscheidenden Beitrag zur Verbesserung der Lebensqualität von unterernährten Patienten und zur Steigerung der Kosteneffizienz medizinischer Maßnahmen beitragen.

\section{Einleitung \\ $\nabla$}

Künstliche Ernährung im ambulanten Bereich umfasst die heimparenterale Ernährung (HPE) und heimenterale Ernährung (HEE) sowie die orale Trinknahrung (ONS), die bei den Patienten zu Hause oder in Pflegeeinrichtungen durchgeführt wird. Die Verfahren wurden in den 70er-Jahren des vergangenen Jahrhunderts etabliert und haben sich inzwischen zu einer sicheren und bewährten medizinischen Maßnahme entwickelt, die sich nicht zuletzt aufgrund der zunehmenden Ambulantisierung der Medizin stetig verbreitet. Meistens beginnen HPE/HEE im Anschluss an einen stationären Aufenthalt in einer Klinik. Aus den Indikationen zur HPE und HEE ergibt sich, dass die Patienten vielfach aus dem onkologischen oder geriatrischen Bereich kommen, seltener aus dem gastroenterologischen oder viszeralchirurgischen Sektor. Deshalb ergeben sich insbesondere Überschneidungen mit den DGEM-Leitlinien „Klinische Ernährung in der Geriatrie“ [1], „Klinische Ernährung in der Neurologie“ [2] und „Klinische Ernährung in der Onkologie“ (in Vorbereitung).

Ziel einer künstlichen Ernährung ist die Verbesserung des Ernährungszustands bzw. das Vermeiden einer Verschlechterung derselben sowie die Verbesserung oder Stabilisierung der subjektiven Lebensqualität. Das gilt sowohl für den stationären als auch für den ambulanten Bereich. Um diese Ziele zu erreichen, sind neben Indikation und Kontraindikation spezielle Durchführungsempfehlungen unter ambulanten Bedingungen zu beachten. Sie sind teilweise ähnlich wie im stationären Bereich, woraus sich Überschneidungen mit der DGEM-Leitlinie „Besonderheiten der Überwachung bei künstlicher Ernährung“ [3] u.a. Leitlinien ergeben. Auf die Elimination solcher Überschneidungen wurde bewusst verzichtet, weil die einzelnen Leitlinien ihre Eigenständigkeit bewahren sollen, damit sie unabhängig voneinander lesbar bleiben.

Die Aspekte Prognose, Lebensqualität und Ethik haben im ambulanten Bereich einen besonders hohen Stellenwert. Darauf wird in den Kommentaren immer wieder eingegangen. Deshalb erge- nursing standards in order to achieve a high hygiene quality. For the exclusive and long-term HEN via gastrointestinal tube and for nutritional support by ONS nutritionally complete formula ("balanced diets") should be used because they meet the D-A$\mathrm{CH}$ recommendations and EU guidelines. For HPE, all-in-one bags/three-chamber-bags should be used because these are considered as safe, effective and risk-reducing standards for HPN.

Conclusion: HEN and HPN are accepted as safe procedures, if they are performed following standardized routines according to upto-date scientific evidence, and if they are coordinated preferably by an interdisciplinary nutrition support team (NST). The NST can enhance the quality of treatment and reduce complication rates. Nutritional support in the homecare and outpatient sector can contribute substantially to an improvement of the patient's quality of life and of cost efficiency of medical procedures.

ben sich auch Überschneidungen mit der DGEM-Leitlinie „Ethische und rechtliche Gesichtspunkte der künstlichen Ernährung“ [4].

Voraussetzung für die Durchführung einer künstlichen Ernährung im ambulanten Bereich ist eine detaillierte Erläuterung von potenziellem Nutzen und Risiken solcher Maßnahmen durch einen Arzt oder eine entsprechend geschulte Ernährungsfachkraft. Der Betroffene sollte nicht nur sein Einverständnis erklären, sondern aktiv die Durchführung der geplanten Ernährungsmaßnahmen wünschen. Eine weitere Voraussetzung ist die Wahl eines für den ambulanten Bereich geeigneten Zugangs und dessen fachgerechte Pflege. Die Komplexität der Betreuung von Patienten mit künstlicher Ernährung, insbesondere im ambulanten Bereich, begründet eine klar strukturierte Organisation sowie eine Betreuung möglichst durch ein Ernährungsteam, das interdisziplinär aus geeigneten Fachkräften besetzt ist. Auf die Vorteile eines solchen Ernährungsteams, das Kosten und Komplikationen nachweislich senken kann, wird besonders eingegangen.

\section{Methodik}

$\nabla$

Während das Thema ambulante künstliche Ernährung in den früheren Leitlinien nur für den parenteralen Bereich separat adressiert wurde [5], deckt die aktuelle Leitlinie sowohl die HEE als auch die HPE und die Themen Organisation und Ernährungsteam ab. Die vorliegende Leitlinie orientiert sich an internationalen Leitlinien von europäischen Nachbarn, ESPEN und ASPEN [6 -9] sowie anderen evidenzbasierten Empfehlungen, die kürzlich publiziert wurden [10].

Es handelt sich hierbei um eine S3-Leitlinie der DGEM (AWMFRegisternummer 073/021). Die Methodik ist im Leitlinienreport ausführlich beschrieben, wo sich auch die Suchstrategien und Evidenztabellen finden. Der Leitlinienreport ist über die Internetseite der Arbeitsgemeinschaft der Wissenschaftlichen Medizinischen Fachgesellschaften e.V. (AWMF) abrufbar (www.awmf. 
org, AWMF-Registernummer der Fachgesellschaft 073). Ein Auszug zum methodischen Vorgehen bei der Leitlinienerstellung wurde bereits in der Aktuellen Ernährungsmedizin veröffentlicht [11].

Neben dem Empfehlungsgrad wird auch die Outcome-Bewertung bei den Empfehlungen mitangegeben (Biomedizinische Endpunkte [BM], Patientenzentriertes Outcome [PC], Gesundheitsökonomische Parameter [HE], Medizinische Entscheidungsfindung [DM], Mehr-Komponenten-Outcome-Modelle [MC]) [12].

\section{Indikation \\ $\nabla$ \\ Empfehlung 1:}

Eine künstliche Ernährung im ambulanten Bereich soll durchgeführt werden, wenn entweder Mangelernährung nachgewiesen ist bzw. droht oder die orale Nahrungsaufnahme relevant eingeschränkt ist und wenn es dadurch innerhalb der zu erwartenden Lebenszeit des Betroffenen zu einer relevanten Verschlechterung des Ernährungszustands, der Prognose oder der Lebensqualität kommt und wenn solche Verschlechterungen nicht durch andere Maßnahmen (z. B. Behandlung der Grunderkrankung) behoben werden können. Die Indikation muss von einem Arzt gestellt werden.

[KKP; starker Konsens]

Kommentar: Die Indikation zur künstlichen Ernährung im ambulanten Bereich unterscheidet sich nicht grundsätzlich von der Indikation im stationären Bereich, wobei die Aspekte Prognose, Lebensqualität und Ethik (vgl. Empfehlung 3 und DGEM-Leitlinie „Ethische und rechtliche Gesichtspunkte der künstlichen Ernährung“ [4]) im ambulanten Bereich einen größeren Stellenwert haben als im stationären Bereich. Die Kriterien für Mangelernährung werden im DGEM-Leitlinienteil „DGEM-Terminologie in der klinischen Ernährung“ [13] definiert. Relevante Einschränkung der oralen Nahrungsaufnahme bedeutet eine unzureichende Deckung des Bedarfs, d. h. ein Energiedefizit von mehr als $10 \mathrm{kcal} / \mathrm{kg}$ Körpergewicht oder etwa $500 \mathrm{kcal} /$ Tag über voraussichtlich mehr als 7 Tage $[14,15]$. Eine Einschränkung der Nahrungsaufnahme ist beispielsweise anzunehmen, wenn trotz fachgerechter Ernährungsberatung und Behandlung von Anorexie, gastrointestinalen Störungen, Schmerzen und psychosozialem Stress die normale Nahrungsaufnahme unter dem individuellen Bedarf liegt. Wenn diese Kriterien erfüllt sind, soll umgehend und nicht erst nach 7 Tagen mit der Durchführung der künstlichen Ernährung im ambulanten Bereich begonnen werden. Relevante Verschlechterung des Ernährungszustands ist anzunehmen, wenn ein Verlust von 5-10\% des Körpergewichts eingetreten ist [16]. Der Ernährungszustand wird sich rascher verschlechtern, wenn die Nahrungsaufnahme stark (auf 25\% des Bedarfs) eingeschränkt ist als wenn die Einschränkung moderat (auf 50\% des Bedarfs) oder gering (auf 75\% des Bedarfs) ist. Eine relevante Verschlechterung des Ernährungszustands wird früher erreicht bei Personen, die bereits vorher Gewicht verloren hatten im Vergleich zu Personen mit zuvor stabilem Gewicht. Der Ernährungszustand wird sich rascher verschlechtern, wenn katabole Prozesse aktiviert sind (z.B. bei Infektionen oder systemischer Entzündung) oder wenn gleichzeitig belastende Therapien (z. B. Chemotherapie) durchgeführt werden [17]. Bei ambulanter Durchführung einer Sonden- oder intravenösen Ernährung muss gesichert sein, dass die erforderlichen technischen Maßnahmen zur Vorberei- tung und Applikation der Nährlösungen langfristig und sicher durchgeführt werden können.

\section{Empfehlung 2:}

Bei Vorliegen einer chronischen Erkrankung, die mit einem erhöhten Risiko für Mangelernährung, Schluckstörung oder Appetitverlust assoziiert ist, sollte frühzeitig eine künstliche Ernährung im ambulanten Bereich erwogen werden.

[KKP; starker Konsens]

Kommentar: Beispiele für den Einsatz von HEE sind Tumorkachexie und tumorbedingte Kau- und Schluckstörungen, neurologische Erkrankungen (ischämischer Hirninsult, Depression u.a.), Schluckstörungen im Rahmen von Erkrankungen anderer Genese und zystische Fibrose. Beispiele für den Einsatz von HPE sind Tumorleiden (besonders bei Magen-, Leber-, Pankreas-, Kolon- und Ovarialtumoren mit Peritonealkarzinose oder funktionell relevantem Befall der großen abdominellen Gefäße; auch in Situationen ohne kuratives Therapieziel) und das Kurzdarmsyndrom (als Folge eines Mesenterialinfarkts, chronisch-entzündlicher Darmerkrankungen, Tumoren oder Strahlenschäden).

Unter den neurologischen Erkrankungen wurde die Demenz nicht berücksichtigt, weil diese nicht typischerweise mit HEE oder HPE assoziiert ist.

\section{Empfehlung 3:}

Eine künstliche Ernährung im ambulanten Bereich soll nicht durchgeführt werden, wenn die Lebenserwartung voraussichtlich geringer als ein Monat ist, wenn die Maßnahme durch den Patienten oder dessen gesetzlichen Betreuer abgelehnt wird, wenn unzureichende Compliance zu erwarten ist oder wenn nicht überwindbare organisatorische bzw. logistische Einschränkungen bestehen. [KKP; starker Konsens]

Kommentar: Auch wenn die Einschätzung der mutmaßlichen Lebenserwartung schwierig ist, sollte sie mit geeigneten Methoden im Verlauf versucht werden [18]. Weitere Informationen zum palliativmedizinischen Konzept und weiterführende Literatur finden sich in der DGEM-Leitlinie „Ethische und rechtliche Gesichtspunkte der künstlichen Ernährung“ [4].

\section{Empfehlung 4:}

Beim absehbaren Übergang aus der Palliativsituation in die Sterbephase sollen Therapieziele der künstlichen Ernährungstherapie im Sinne der Symptomkontrolle neu definiert werden. Die Entscheidung bezüglich der Fortsetzung bzw. der Beendigung der künstlichen Ernährungstherapie soll der betreuende Arzt gemeinsam mit dem Patienten und den Angehörigen nach den Grundsätzen der deutschen Bundesärztekammer ${ }^{1}$ treffen.

[KKP; starker Konsens]

Kommentar: Weil der Beginn der Sterbephase wissenschaftlich nicht weiter objektivierbar ist, kann eine ernährungsmedizinische Intervention in dieser Lebensphase keinem evidenzbasierten Algorithmus folgen. Die Indikation zu einer künstlichen $\mathrm{Er}$ -

\footnotetext{
${ }^{1}$ Grundsätze der Bundesärztekammer zur ärztlichen Sterbebegleitung vom 17.02.2011: http://www.bundesaerztekammer.de/page.asp?his=0.6. 5048.5049
} 
nährung ist jetzt mit einer sorgfältigen, individuellen Abwägung möglicher Risiken und Nutzen unter der neuen Zielsetzung der Sterbebegleitung zu stellen [19-22]. Siehe hierzu auch DGEMLeitlinie „Ethische und rechtliche Gesichtspunkte der künstlichen Ernährung“ [4] und „Klinische Ernährung in der Neurologie“" [2].

\section{Empfehlung 5:}

Die Indikation für die Fortsetzung der HEE und HPE sollte in regelmäßigen Abständen überprüft werden. Das zeitliche Intervall für diese Überprüfung ergibt sich aus der Art der Grunderkrankung und deren Verlauf.

[B; starker Konsens]

Kommentar: Die empfohlenen Abstände zur Überprüfung variieren je nach Phase der künstlichen Ernährung und sollten zumindest einmal pro Monat in den ersten 3 Monaten und danach quartalsweise erfolgen [23]. Eine HPE kann beendet werden bzw. auf HEE umgestellt werden, wenn der Energiebedarf oral oder enteral gedeckt werden kann. Eine HEE kann in der Regel beendet oder auf orale Trinknahrung (ONS) umgestellt werden, wenn das Energiedefizit weniger als $500 \mathrm{kcal} /$ Tag beträgt und das Körpergewicht ohne HEE stabil bleibt [14, 15, 24]. Bei geriatrischen Patienten kann diese Grenze nicht immer zugrunde gelegt werden. Jedenfalls ist im Verlauf zu prüfen, ob der Ernährungszustand nach Beendigung der künstlichen Ernährung stabil bleibt. Deshalb sollte die Ernährungssonde (i.d.R. die PEG) zunächst für mindestens einen weiteren Monat behalten und nur stillgelegt werden, bis die orale Zufuhr dauerhaft gesichert ist. Bei Patienten, die zwischenzeitlich ein funktionstüchtiges Dünndarmimplantat erhalten haben bzw. erfolgreich Dünndarm transplantiert werden konnte, kann die künstliche Ernährung in der Regel eingestellt werden. Trotz eingeschränkter Studienlage hat sich die Expertengruppe aufgrund der eindeutigen Sachlage für den Empfehlungsgrad B entschieden.

\section{Zugangswege und Pumpen}

\section{Empfehlung 6:}

Der geeignete Zufuhrweg für eine ambulante künstliche Ernährung hängt von der funktionellen Integrität des Magen-Darm-Trakts ab: Bei Fehlen relevanter Einschränkungen der Magen-Darm-Funktion oder isolierten Kaustörungen sollen orale Trinknahrungen (ONS) eingesetzt werden. Bei Schluckstörungen sowie bei relevanten Passagestörungen des Ösophagus oder Magens sollte eine Sondenernährung eingesetzt werden. Bei relevanten Störungen der Transport- oder Absorptionskapazität des Dünndarms soll eine intravenöse Ernährung gewählt werden. Kontraindikationen des gewählten Zufuhrwegs sollen beachtet werden.

[KKP; starker Konsens]

\subsection{Heimenterale Ernährung}

\section{Empfehlung 7:}

Bei einer längerfristigen HEE soll eine perkutane endoskopische Gastrostomie (PEG)-Sonde, gegebenenfalls eine perkutane endoskopische Jejunostomie (PEJ) erwogen werden.

[A; starker Konsens]
Die PEG sollte gegenüber den herkömmlichen chirurgischen Gastrostomie-Verfahren vor allem aufgrund einer niedrigeren Komplikationsrate bevorzugt werden.

[B; starker Konsens]

Kommentar: Fünf Studien, davon 4 prospektive und 3 randomisierte, vergleichen PEG-ernährte und nasogastral-ernährte Patienten und belegen eindeutig die Vorteile einer PEG-Ernährung [25 - 29]. Der Vorteil der PEG ist damit begründet, dass sie gegenüber einer nasogastralen Ernährung die Aufnahme größerer Nahrungsmengen [26, 27] über längere Zeiträume [26-29] erlaubt, weniger Behandlungsprobleme wie Selbstentfernungen [22] und Sondenneuanlagen [25, 27, 29] verursacht und damit den Ernährungszustand verbessert [26-28, 30]. Außerdem werden bei PEG-Patienten weniger Fixierungen (7 vs. $22 \%$ ) sowie eine subjektive Pflegeerleichterung für das Pflegepersonal und eine bessere Lebensqualität für die Patienten beschrieben [25]. In der Cochrane-Analyse von Gomes et al. [31] konnte in 9 randomisiert-kontrollierten Studien allerdings kein Unterschied in der Mortalität PEG-ernährter und nasogastral-ernährter dysphagischer Patienten festgestellt werden.

Eine geringere Aspirationshäufigkeit per PEG versus nasogastraler Sonde beschreiben Dwolatzky et al. [28] und Fay et al. [29]. Die Cochrane-Analyse von Gomes et al. [31] zeigt jedoch keinen Unterschied der beiden Ernährungsarten bezüglich Pneumonie bei dysphagischen Patienten. Die häufige Kombination von neurogener Schluckstörung mit kognitiver Einschränkung bei geriatrischen Patienten (Demenz, Parkinson, rezidivierende zerebrale Insulte) ist mit einem erhöhten Aspirationsrisiko verbunden. In diesen Situationen ist eine frühzeitige PEG-Anlage gegenüber einer nasogastralen Sondennahrung höchstwahrscheinlich von Vorteil.

Beim Auftreten von gastroduodenalen Motilitätsstörungen, Magenausgangsstenosen oder Aspirationen kann konsekutiv auch eine PEG/J (PEG mit einem jejunalen Schenkel) oder primär eine PEJ angelegt werden [32-36].

Die PEG hat die herkömmlichen chirurgischen Gastrostomie-Verfahren, vor allem aufgrund einer niedrigeren Komplikationsrate, weitgehend verdrängt. Tatsächlich ist die Überlegenheit einer enteralen Langzeiternährung via PEG-/PEJ-Sonde gegenüber der Ernährung über nasogastrale bzw. nasojejunale Sonden oder operativ angelegte Gastrostomien (z. B. nach Witzel oder Stamm) durch klinische Studien eindeutig belegt [25-27, 32, 37, 38].

\subsubsection{Pflege und Umgang mit der Sonde}

\section{Empfehlung 8:}

Die Sondeneintrittsstelle sollte regelmäßig und sorgfältig beobachtet, wie eine Wunde behandelt und in den ersten 5-7 Tagen unter aseptischen Bedingungen verbunden werden.

[B; starker Konsens]

Bei abgeheilter Wunde und reizlosen Wundverhältnissen kann der Verbandswechsel 2-mal wöchentlich erfolgen

[C; starker Konsens]

oder - im Ermessen des Arztes - ganz auf einen Verband verzichtet werden.

[KKP; starker Konsens]

Kommentar: Slater [39] schlägt vor, die Eintrittsstelle der perkutan eingelegten Sonde während des ersten Tages alle 4 Stunden auf Rötung, Schwellung oder Austritt von Flüssigkeit zu untersuchen. Gemäß den Europäischen Empfehlungen von ESPEN kann 
die Eintrittsstelle aber auch im Rahmen einer täglichen Wundversorgung ein erstes Mal begutachtet werden [32]. Nach der Sondenanlage hat es sich bewährt, die Eintrittstelle mit einer beschichteten absorbierenden Wundauflage zu versorgen. Nach der Abheilung soll eine sterile trockene Abdeckung erfolgen. Eine regelmäßige Reinigung im Rahmen des Verbandswechsels mit geeigneten Materialien ist zu empfehlen [40]. Ein bakteriostatisch wirksamer Glycogel-Verband bei neuangelegter PEG führt nicht zu weniger Infektionen als ein trockener herkömmlicher Wundverband und kann somit nicht generell empfohlen werden [41]. Zur Desinfektion der abgeheilten Eintrittsstelle sind Lösungen auf der Basis von Polyhexanid oder Alkohol geeignet [32]. Hierbei sind die Zeiten für Wirksamkeit und Trocknung einzuhalten. Von einer wiederholten Anwendung von toxischirritativen Substanzen in der Sondenumgebung ist ebenfalls abzusehen, auch um das Sondenmaterial nicht zu schädigen (z.B. jodhaltige Mittel). Den betreuenden Pflegepersonen zu Hause sollten jederzeit schriftliche Anweisungen zur Verfügung stehen, um eine einheitliche, qualitativ hochstehende Pflege zu garantieren und klar verständliche Anweisungen an die Patienten abzugeben $[42,43]$.

\section{Empfehlung 9:}

Die PEG-Sonde soll anfänglich adaptiert an die Bauchwand mit geringem Spiel fixiert werden.

[KKP; starker Konsen]

Eine PEG soll regelmäßig mobilisiert werden.

[KKP; starker Konsens]

Bei der Fixierung der äußeren Halteplatte soll auf das Niveau zur

Haut geachtet werden, welches bei der Anlage erreicht wurde.

[KKP; starker Konsens]

Allerdings dürfen eine PEG mit jejunaler Sonde bzw. „RIG-Sonden“

nicht mobilisiert werden.

[C; starker Konsens]

Kommentar: Das früher übliche Vorgehen, die PEG-Sonde während der ersten 24 Stunden nach Einlage unter Zug zu stellen und damit die Magenwand an die Bauchwand zu pressen, wird heute nicht mehr empfohlen. Allerdings muss auch vermieden werden, dass die Platte im Magen zu wenig fest verankert ist, damit sich ein Kanal um die Sonde herum bilden kann [32, 44]. Um ein Einwachsen zu verhindern, sollte die Sonde während der täglichen Kontrolle der Einstichstelle vorsichtig mobilisiert werden, indem sie nach innen bewegt und gedreht wird. Bei jejunalen Sonden, Sonden in Direktpunktionstechnik oder Ballonsonden sollten die Systeme nach Maßgaben des Herstellers bzw. des Operateurs abgedeckt werden [32, 45]. Durch das tägliche Mobilisieren und Rotieren um die eigene Achse wird das Risiko eines Einwachsens der inneren Halteplatte (Burried-Bumper-Syndrom) deutlich vermindert. Deshalb soll die äußere Halteplatte immer wieder vorsichtig gelöst und gereinigt werden [46]. Bei jejunaler Lage darf die Sonde weder mobilisiert noch rotiert werden, weil sonst die Gefahr der Schlingen- oder Knotenbildung besteht [47]. Unter radiologischer Kontrolle angelegte Gastrostomien (Radiologically-inserted gastrostomy, RIG) dürfen nicht rotiert werden, weil der interne „Pigtail“ durch einen Faden, der durch die Sonde führt, aufrechterhalten wird. Deshalb sollte Zug auf die Sonde vermieden werden. Die Gefahr eines Burried-Bumper-Syndroms besteht nicht [48].

\section{Empfehlung 10:}

Der Beginn der enteralen Ernährung kann bereits wenige Stunden nach Anlage einer PEG ohne Risiko erfolgen.

[A; starker Konsens]

Ein stufenweiser Kostaufbau mit einer adäquaten Flüssigkeitsmenge sollte angestrebt werden.

[B; starker Konsens]

Kommentar: Die Empfehlung beruht auf einer Metaanalyse [49], einer offenen kontrollierten Studie [50] und knüpft an bestehende Leitlinen an [6]. Dabei wurde festgestellt, dass ein Beginn mit Sondenernährung, z.B. innerhalb von $4 \mathrm{~h}$ nach Anlage, nicht mehr Komplikationen verursacht als ein verzögerter Start nach 6-12 h oder in den Folgetagen. Daraus ist abzuleiten, dass ein Start der Sondenernährung bereits ab $3 \mathrm{~h}$ nach Anlage ohne erhöhte Gefahrenrate erfolgen kann. Voraussetzung ist, dass die Sondenanlage komplikationslos erfolgte. Generell soll der Kostaufbau nach Erstanlage langsam erfolgen, mit einer adäquaten Flüssigkeitsmenge (30-40 mL/kg Körpergewicht/Tag) und Ballaststoffen/Nahrungsfasern [45].

\section{Empfehlung 11:}

Zur Vermeidung von Sondenkomplikationen sollte die Ernährungssonde für HEE vor und nach Gebrauch gründlich mit frischem Trinkwasser gespült werden.

[B; starker Konsens]

Dies gilt besonders auch vor und nach Applikation von Medikamenten über die Sonde, was grundsätzlich nur bei fehlenden Alternativen erfolgen kann und bei festen Arzneiformen eine geeignete Vorbehandlung derselben erfordert.

[C; starker Konsens]

Bei längerem Nichtgebrauch einer Sonde kann empfohlen werden, die Sonde in der Regel 2-mal täglich mit frischem Trinkwasser zu spülen.

[C; starker Konsens]

Kommentar: Die Komplikationsrate nach endoskopischer Einlage von Ernährungssonden wird in der aktuellen Literatur mit $8-30 \%$ angegeben, wobei schwere, therapiebedürftige Komplikationen in etwa 1-4\% der Fälle auftreten [32, 37, 51-53]. Zu den Komplikationen, die häufig bei PEG/PEJ in der Langzeiternährung auftreten, gehören vor allem Materialprobleme, Obstruktionen und lokale Wundinfekte. In der Befragung von Crosby et al. [54] wurden 55 Patienten - mit einer durchschnittlichen Dauer der HEE von 25,9 Monaten - nach mechanischen Komplikationen gefragt: $56 \%$ berichteten über eine gebrochene oder auslaufende Sonde und 29\% der Gruppe hatten bereits Sondenverstopfungen erlebt. Zu einem ungewollten Herausziehen einer Sonde kommt es laut einer Untersuchung aus den USA bei rund 4,1\% von 563 PEGs während der ersten 7 Tage und in 12,8\% während der ganzen Lebensdauer der PEG, wobei es in nur 3 Fällen zu schweren gesundheitlichen Komplikationen durch das unbeabsichtigte Herausziehen der Sonde kam [55].

Nach jeder Nutzung der Sonde muss diese mit ca. $40 \mathrm{~mL}$ frischem Leitungswasser mit Trinkwasserqualität bzw. Trinkwasser gespült werden, um einer Krustenbildung oder Verstopfung vorzubeugen [32]. Ein ausgiebiges Spülen der Sonde ist besonders nach Gabe von Medikamenten über die Sonde wichtig [56]. Wasser ist dabei pankreasenzymhaltigen kommerziellen Reinigungsmitteln wegen potenziellen Ausflockungen (Verstopfen) vorzuziehen, aber 
auch aus Kosten- und Hygienegründen von Vorteil [57]. Zum Spülen ist Wasser dem Tee vorzuziehen, denn Tee kann durch seine Gerbstoffe Sondenverfärbung sowie Inkompatibilitäten mit Nahrungsbestandteilen oder Medikamenten bewirken. Ob bei jejunaler Sondenlage statt Wasser besser isotonische $\mathrm{NaCl}$-Lösung verwendet werden soll, ist nicht belegt, aber naheliegend.

Eine Sondenobstruktion ist praktisch immer Folge einer mangelnden Langzeitpflege der Sonden und nur selten der Materialermüdung. Die Ursache liegt meist in der Applikation falscher oder nicht ausreichend zerkleinerter Medikamente über die Sonde und dem unzureichenden Nachspülen der Sonde nach Medikamentengabe. Die Eignung der Medikation ist durch den verordnenden Arzt im Voraus in Absprache mit dem Pharmazeuten zu überprüfen: Lösliche Tabletten sollten vorab in $15-30 \mathrm{~mL}$ Wasser aufgelöst werden, Medikamente in Tropfen- oder Sirupform gut geschüttelt werden und dickflüssige Lösungen mindestens 1:1 (vgl. Osmolalität unten) mit frischem Trinkwasser verdünnt werden; überzogene und nicht überzogene Tabletten sollten erst mittels eines Mörsers zerkleinert werden, falls die Stabilität und Aufnahme des Wirkstoffs dies erlaubt, und dann in 15$30 \mathrm{~mL}$ aufgelöst werden; Kapseln sollten, falls dies nötig und erlaubt ist, geöffnet und der Kapselinhalt, je nach Teilchengröße, gemörsert werden [58, 59]. Bei flüssigen Arzneimittelformen sind der pH und die Osmolalität (max. 500-600 mOsmol/kg) zu berücksichtigen, um Verklumpungen oder unerwünschte Wirkungen wie Diarrhöen zu vermeiden [58]. Die kontinuierliche enterale Ernährung sollte vor der Applikation des Arzneimittels gestoppt werden. Jedes Medikament sollte separat appliziert werden. Zwischen der Verabreichung verschiedener Medikamente sollte jeweils mit mindestens 5-10 mL frischem Trinkwasser gespült werden. Vor und nach Applikation von Medikamenten wie auch vor und nach Verabreichung von Sondenkost sollte die Sonde mit 20-40 mL frischem Trinkwasser gespült werden [58].

Bei einer bereits eingetretenen Verstopfung der Sonde, z. B. durch Medikamente sollte diese zunächst mit Wasser gespült werden. In Ausnahmefällen können kohlensäurehaltige Getränke, Pepsinwein oder eine Suspension aus Pankreas-Enzympräparat und Natriumhydrogenkarbonat benutzt werden [60]. Die Zutaten für den Pepsinwein sind $30 \mathrm{~mL}$ Malaga (Süßwein Malvasia) und $75 \mathrm{mg}$ Pepsin. Die beiden Komponenten werden gut miteinander vermischt. Bei Erwachsenen 1 - $5 \mathrm{~mL}$ Pepsinwein in die Sonde injizieren, je nach Erfolg mehrmals wiederholen oder den Pepsinwein 5-10 Minuten einwirken lassen (Pepsin löst Eiweiß und Alkohol löst Fett). Die Haltbarkeit des Pepsinweins im Kühlschrank beträgt 14 Tage. Kleinere Spritzenvolumina $(<10 \mathrm{~mL})$ sollten gemieden bzw. mit besonderer Vorsicht verwendet werden, da es dabei zu einer hohen Druckentwicklung mit Ruptur der Sonde kommen kann [61].

Ältere Patienten brauchen zur HEE meist ausführlichere Informationen und intensivere Betreuung, um solche Komplikationen zu vermeiden [62]. Besondere Maßnahmen müssen auch bei Patienten ergriffen werden, die eine PEG ambulant erhalten und demzufolge hauptsächlich auf eine Schulung außerhalb des Krankenhauses angewiesen sind $[63,64]$.

Bei Risikopatienten sollte zur Vermeidung lokaler Infekte bei der PEG-Anlage eine systemische Antibiotikaprophylaxe erfolgen, um die Infektionsrate zu reduzieren. Einen Konsens für eine generelle Antibiotikaprophylaxe gibt es allerdings nicht [32]. Im Zweifelsfall sollte eher eine großzügige Indikationsstellung für die Durchführung einer individuellen Antibiotikaprophylaxe erfolgen, da die gezielte systemische Antibiotikatherapie die Infektionsrate signifikant reduzieren kann [65]. Eine einmalige Anti- biotikaprophylaxe entfällt, wenn der Patient aus anderen Gründen zeitgleich eine antibiotische Therapie bekommt [32].

\subsection{Heimparenterale Ernährung}

\section{Empfehlung 12:}

In Abhängigkeit der voraussichtlichen Dauer der HPE sollte der Kathetertyp, die Zugangstechnik und die Katheterposition mit dem geringsten Komplikationsrisiko (infektiös und nicht infektiös) gewählt werden.

[KKP; starker Konsens]

Für die Langzeit-HPE, v. a. für ambulante Patienten zu Hause, sollten subkutan tunnelierte Katheter oder implantierte Portsysteme zur Anwendung kommen.

[B; starker Konsens]

Kommentar: Wegen der hohen Osmolalität ( $\geq 800 \mathrm{mOsmol} / \mathrm{kg}$ ) der zu Hause verwendeten Nährmischungen muss die HPE i.d.R. über einen zentralvenösen Zugang erfolgen. Hinsichtlich der Insertionsstelle ist die Studienlage nach wie vor begrenzt [66]. Perkutan eingelegte Katheter sollten in der Regel in der V. cava superior platziert werden. Bei Erwachsenen ist die V. subclavia aus infektiologischer Sicht der V. jugularis interna und anderen $\mathrm{Zu}-$ gangswegen vorzuziehen [67-70]. Der Katheter für den zentralvenösen Zugang sollte so dünn wie möglich, die entsprechende Vene möglichst großlumig sein. Bei den zentralen Verweilkathetern steigt mit zunehmender Lumenzahl die Infektionsrate [7174]. Es besteht eine enge Korrelation zwischen Liegedauer und Infektionsrisiko [75, 76] des zentralvenösen Zugangs. Auch thrombotische Komplikationen hängen von der Liegedauer ab [69, 77]. Für eine langfristige HPE (>3 Wochen) eignen sich tunnelierte oder implantierte Dauerkatheter (Broviac- oder Hickman/Groshong-Katheter, Portsysteme) [78]. Broviac- und Hickman/Groshong-Katheter sind implantierbare venöse Silikonkatheter, die perkutan ausgeleitet werden. Vor der Austrittsstelle ist der Katheter von einer Halterung aus Kunststoff umgeben, in die das Körpergewebe einwachsen und dadurch das Eindringen von Bakterien von außen verhindern kann [79]. Aufgrund der Relevanz des Themas, des hohen Konsens in der Expertengruppe und der limitierten Möglichkeit für die Durchführung weiterer Studien aufgrund ethischer Gesichtspunkte wurde der Empfehlunsgrad des zweiten Satzes von C nach B hochgestuft.

In der Studie von Verseleijen et al. [80] wird als mögliche Alternative zu den bereits vorgestellten Systemen die Einlage eines zentralvenösen Katheters in einer arteriovenösen Fistel für die Patienten mit einer HPE vorgeschlagen. Diese Methode zeigt eine geringere Komplikationsrate von schweren Infektionen (Kathetersepsis) gegenüber der konventionellen Lage (V. subclavia, V. jugularis) des zentralvenösen Katheters.

Bei richtiger Handhabung durch die Patienten wurde eine sehr gute Langzeitverwendbarkeit über mehrere Jahre und eine hohe Patientenakzeptanz beobachtet. Die Erfahrung des Teams in der Zusammenarbeit mit den Chirurgen (Katheterwahl und -einlage) und dem Kathetermanagement ist von großer Bedeutung [81].

\section{Empfehlung 13:}

Bei HPE über ein Portsystem sollte der Portnadelwechsel bei täglicher parenteraler Ernährung alle 3-7 Tage erfolgen.

[B; starker Konsens]

Bei intermittierender Ernährung über ein Portsystem sollte die Kanüle für die infusionsfreie Zeit entfernt werden.

[KKP; starker Konsens] 
Kommentar: Portsysteme sind total implantierbare venöse Silikon- oder Polyurethankatheter mit subkutan versenkter Reservoirkammer aus Titan oder Keramik. Die Portmembran besteht aus Silikon und darf nur mit speziellen Portkanülen (nicht stanzenden Nadeln) punktiert werden. Bei HPE-Patienten mit zyklischer Nahrungsapplikation wird der Portnadelwechsel jeden 3. - 7. Tag empfohlen, die Verschlussdeckel sollten in gleichen Abständen ersetzt werden [82 - 86]. Raad et al. [86] fanden eine höhere Kontaminationsrate an Portnadelschlauchsystemen, die nur alle 4-7 versus alle 3 Tage gewechselt wurden. Eine höhere Infektionsrate wurde allerdings nicht gezeigt. In einer retrospektiven deskriptiven Studie von Chang et al. [82] wurde eine geringere Infektionsrate beim Wechsel der Portnadel alle 3 versus alle 7 Tage beobachtet. Falls keine Nährlösung und nur Medikamente (Zytostatika) über den Port appliziert werden, kann die Portnadel in der Regel über 2 Wochen in situ belassen werden [84-86]. Insgesamt lässt die Studienlage darauf schließen, dass durch das Zeitintervall des Portnadelwechsels von 3 Tagen das katheterassoziierte Infektionsrisiko vermindert werden kann. Noch größere Bedeutung als dem Zeitintervall kommt jedoch einem hohen Hygienestandard zu. Bei Risikopatienten wie unter Immunsuppression und bei katheterassoziierten Infekten in der Vorgeschichte sollte ein kürzeres Intervall bevorzugt werden.

\subsubsection{Pflege und Umgang mit den Zugangswegen}

\section{Empfehlung 14:}

Die Durchführung eines Verbandswechsels an einem zentralen Venenkatheter soll unter der Voraussetzung ausreichender Händehygiene und aseptischer Arbeitsweise erfolgen.

[KKP; starker Konsens]

Kommentar: Studien zu hygienischen Verbandswechseln an Zentralvenenkathetern wurden vorwiegend im stationären Bereich durchgeführt, die Ergebnisse sind aber auch für den ambulanten Bereich relevant und sollten dort berücksichtigt werden. Abhandlungen über die möglichen, evidenzbasierten präventiven Maßnahmen zur Verhinderung von katheterbedingten Infekten bei PE wurden von Attar et al. [87] und O'Grady et al. [75] publiziert. Auch beim Umgang mit dem ZVK ist die Bedeutung der Schulung des Betreuungsteams entscheidend, wobei der Patient selbst oder direkt betreuende Angehörige mitgeschult werden müssen, da sie für die längerfristige Verhinderung solcher Infekte entscheidend sind $[75,88,89]$.

\section{Empfehlung 15:}

Zur Desinfektion der Kathetereintrittsstelle sollen chlorhexidinhaltige Mittel verwendet werden.

[A (BM); starker Konsens]

Kommentar: Der Nachweis der Effektivität von Chlorhexidin zur Prävention bakterieller Katheterinfekte wurde an chirurgischen Intensivpatienten [90], allgemeinen Intensivpatienten [91] und pädiatrischen Intensivpatienten [92] nachgewiesen. Diese Ergebnisse wurden in einer kürzlichen Metaanalyse bestätigt [93]. Die Empfehlung, beim ZVK-Verbandswechsel bevorzugt alkoholische Hautdesinfektionsmittel einzusetzen (schnelle und gute Desinfektionswirkung), muss mit den eindringlichen Warnungen mehrerer Katheterhersteller vor möglichen Materialveränderungen (Katheterbrüche) abgewogen werden [90]. Auch das Risiko einer lokalen Dermatitis nach Chlorhexidin-Behandlung kann die Anwendung besonders bei Neugeborenen limitieren [92].

\section{Empfehlung 16:}

Zur Abdeckung der Kathetereintrittsstelle sollen sterile Gazen bzw. Mull oder sterile, durchsichtige, semipermeable Folien eingesetzt werden.

[A; starker Konsens]

Bei immunsupprimierten Patienten oder Patienten mit infizierten

Kathetern in der Vorgeschichte sollte z. B. eine chlorhexidinimprägnierte Wundauflage eingesetzt werden.

[KKP; starker Konsens]

Bei stabilen und reizlosen Wundverhältnissen können Mullverbände alle 2 Tage und transparente Verbände alle 6 Tage gewechselt werden.

[C; starker Konsens]

Ausnahmen stellen durchnässte, verunreinigte oder undichte Verbände dar, die bei Bedarf gewechselt werden müssen.

[KKP; starker Konsens]

Kommentar: Eine Metaanalyse bestätigte, dass Gaze- und Folienverband vergleichbare pflegetherapeutische Standards sind [94]. Allerdings können Folienverbände zu feuchten Kammern führen und dadurch theoretisch wieder Infektionen begünstigen [94, 95]. Gut abgeheilte Eintrittsstellen von getunnelten Kathetern brauchen keinen Verband. Bei blutender oder nässender Kathetereinstichstelle sollte vorzugsweise ein Gazeverband verwendet werden [96-100]. Ein durchnässter oder gelockerter Verband muss ersetzt werden $[97,98,101]$.

Beim Duschen muss gewährleistet sein, dass kein Wasser über die Kathetereintrittsstelle läuft; hierfür gibt es spezielle Duschpflaster. Nach dem Duschen sollte ein Verbandswechsel durchgeführt werden (feuchte Kammer vermeiden).

Der prophylaktische Einsatz von antibiotikahaltigen Salben begünstigt eine resistente Flora und sollte somit unterlassen werden $[96,102]$. In Bezug auf katheterassoziierte Infektionen wurde durch Abdeckung mit Gaze oder transparentem Film keine Differenz festgestellt [96].

\section{Empfehlung 17:}

Im Rahmen der HPE sollen nicht benutzte Katheter- oder Portsysteme vor und nach der PE-Applikation mit isotoner NaCl-Lösung gespült werden.

[KKP; starker Konsens]

Heparinhaltige Lösungen sollten dafür nicht verwendet werden.

[B; starker Konsens]

Kommentar: Bei den Spülempfehlungen sind Maßnahmen vor und nach Benutzung und Maßnahmen bei temporärer Nichtbenutzung über Tage bis Wochen zu unterscheiden. Der erste Satz ist klinischer Konsens und kann aus ethischen Gründen nicht mit RCT verifiziert werden. Durch das Spülen des Katheters soll das Risiko des Verstopfens reduziert werden. Wenn der zentralvenöse Katheter temporär nicht benutzt wird, sollte er mindestens monatlich mit isotonischer $\mathrm{NaCl}$-Lösung $(0,9 \%)$ gespült werden. Eine Heparinspülung wird generell nicht empfohlen, da sich kein Vorteil ergibt [103]. Die Empfehlung B für den zweiten Satz der Empfehlung begründet sich auf die Relevanz und die Einmaligkeit der hier zitierten europaweiten Studie. Diese Empfehlung wird weiter untermauert durch die Tatsache, dass langfristige 
Antikoagulation auf Heparinbasis mit einem erhöhten Risiko für heparininduzierte Thrombozytopathien (HIT), Osteoporose und Inkompatibilitäten einhergeht.

\section{Empfehlung 18:}

Zur Reduktion des Thrombose- [C] und des Infektionsrisikos [B] sollten Blutentnahmen aus dem zentralvenösen Zugang vermieden werden.

[starker Konsens]

Kommentar: Blutentnahmen aus dem zentralvenösen Katheteroder Portsystem erhöhen das Risiko für katheterassoziierte Infektionen [96, 104] und möglicherweise auch für mechanische Komplikationen wie Thrombosen [105-107]. In 2 Studien konnte gezeigt werden, dass heparinbeschichtete ZVKs Nachteile gegenüber nichtbeschichteten ZVKs hinsichtlich Thrombogenitätspotenzial aufweisen $[104,105]$.

Das Thromboserisiko ist bei HPE erhöht [108-110], deshalb kann eine niedrig dosierte orale Antikoagulation mit geeigneten Substanzen wie z. B. Warfarin durchgeführt werden. Allerdings wird diese prophylaktische Maßnahme kontrovers beurteilt. Die Prophylaxe mit nieder dosiertem Warfarin zeigte in 3 Studien und in der systematischen Übersicht von Klerk et al. eine Senkung des Thromboserisikos [106, 111-113], hingegen waren die Resultate für Heparin negativ [114]. In der Vergleichsstudie von Bern et al. [107] konnte gezeigt werden, dass bei onkologischen Patienten die präventive orale Therapie mit Warfarin ( $1 \mathrm{mg} / \mathrm{Tag})$ zu einer Reduktion von mehr als $50 \%$ der katheterbedingten Thrombosen führt. Diese Resultate konnten jedoch durch die multizentrische randomisierte placebokontrollierte Studie von Couban et al. in einem größeren Patientenkollektiv nicht bestätigt werden [115].

\section{Empfehlung 19:}

Als erste Maßnahme bei Verstopfungen von Katheter- oder Portsystemen, die zur HPE verwendet werden, soll isotonische $\mathrm{NaCl}$-Lösung instilliert werden.

[KKP; starker Konsens]

Wenn dies erfolglos bleibt, können bei Verstopfung nach Blutent-

nahmen Thrombolytika verwendet werden.

[C; starker Konsens]

Kommentar: Die Katheterokklusion stellt die häufigste nichtinfektiöse Komplikation dar. Eine detaillierte Abklärung der potenziellen Ätiologien, die zur Verstopfung geführt haben, ist von zentraler Bedeutung für die nachfolgende Therapiestrategie [78]. Vor allem nach Blutentnahmen über die Katheter- oder Portsysteme können durch Blutgerinnsel oder durch Fibrinrückstände Verstopfungen auftreten. Bei ZVK-Verstopfungen sollte als erste Maßnahme aspiriert und dann sterile isotonische NaCl-Lösung $(0,9 \%)$ unter leichtem Druck injiziert werden. Dafür sollten $10 \mathrm{~mL}-$ Spritzen verwendet werden, ggf. auch kleinere Spritzen, durch die es allerdings auch zur Katheterruptur kommen kann, wenn ein zu großer Druck erzeugt wird. Bei fehlendem Erfolg ist der Vorgang mehrmals zu wiederholen. Bleibt der Katheter weiterhin verstopft, sollte bei Verdacht auf ein Blutgerinnsel eine Spülung mit Urokinase oder rTPase $(5000 \mathrm{IE} / \mathrm{mL})$ durchgeführt werden, wobei die Thrombolytika 30-60 Minuten einwirken sollen [116-118]. Bleiben diese Maßnahmen erfolglos, muss ein Katheterwechsel vorgenommen werden. In seltenen Fällen kann es trotz der erfolgreichen Spülung passieren, dass winzige Mengen Blut an der Katheterwand haften bleiben und auch durch intensives Spülen nicht zu entfernen sind. Dies ist ein idealer Nährboden für Mikroorganismen und kann zur Besiedlung des Katheter-Systems führen.

Falls keine Blutentnahme über das verstopfte System vorgenommen worden ist, muss mit größter Wahrscheinlichkeit eine Okklusion durch Rückstände der Nährlösungskomponenten angenommen werden. Lipidresiduen können ebenfalls zu ZVK-Verstopfungen führen. Diese entstehen in der Regel nur langsam über Tage. In diesem Fall kann die Instillation von Natriumhydroxid (NaOH/Natronlauge: $0,1 \mathrm{mmol} / \mathrm{mL} ; 0,1 \mathrm{M}$; pH 13) erfolgreich sein $[119,120]$. Die Alkoholspülung (Ethanol 96\%) wird von Silikonkatheterherstellern abgelehnt, da Alkohol deren Oberfläche verändern kann.

Unlösliche Ausfällungen entstehen in Zusammenhang mit der Verabreichung von Medikamenten und Elektrolyten wie beispielsweise Kalzium oder Phosphaten. Die Präzipitate können durch Inkompatibilitäten zwischen den erwähnten Bestandteilen, z.B. durch Bildung unlöslicher Kristalle, entstehen [121]. Von besonderer Bedeutung sind Kalziumphosphat-Präzipitate, die durch verschiedene Eigenschaften der Nährmischung wie die Aminosäurenzusammensetzung, relativer Kalzium- und Phosphatgehalt, Temperatur, $\mathrm{pH}$ usw. beeinflusst werden $[121,122]$. Ein durch unlösliche Präzipitate verstopfter, subkutan implantierter Dauerverweilkatheter kann evtl. durch pH-Veränderungen wieder benutzbar gemacht werden [123-125]. Bikarbonat verursacht zahlreiche Inkompatibilitäten und sollte der Nährmischung grundsätzlich nicht zugemischt werden.

Das Spülen mit Heparinlösungen zeigt keine Vorteile gegenüber isotonem $\mathrm{NaCl}$. Es kann aber wegen dem erhöhten Inkompatibilitätsrisiko an der Entstehung von katheterassoziierten Komplikationen (z.B. Infekte, Verstopfungen) beteiligt sein [103]. Solche Inkompatibilitäten müssen als vermeidbare Medikationsfehler betrachtet werden.

\section{Empfehlung 20:}

Bei Verdacht auf Katheterinfektion bei HPE sollen als erstes Blutkulturen peripher und aus jedem Katheterlumen entnommen werden. [KKP; Konsens]

Unter Beachtung der klinischen Situation sollte eine systemische und intraluminale Antibiotikatherapie möglichst nach Antibiogramm versucht werden.

[B; Konsens]

Bei ausgeprägten lokalen oder systemischen Zeichen eines Infekts (beginnendes Organversagen) und/oder bei Nachweis von katheterinduzierter Bakteriämie mit Problemkeimen (z. B. Candida albicans, Pseudomonas-Stämmen oder Staph. aureus) sollte der ZVK entfernt werden.

[B; Konsens]

Bei Problempatienten kann eine Infektionsprophylaxe mit antimikrobiellen Substanzen (Lock-Therapie) erwogen werden. [C; Konsens]

Kommentar: In einer Metaanalyse von 37 Arbeiten zur HPE fand sich als häufigste Komplikation eine Kathetersepsis mit 0,34 Episoden pro Katheter und Jahr [126]. Die Besiedlung eines ZVK mit Bakterien oder Pilzen ist eine potenziell lebensgefährliche Komplikation der HPE, die eine septische Thrombose, eine Endokarditis oder die Infektionsansiedlung in andere Organe zur Folge haben kann [127]. Im stationären Bereich kommt die katheterbe- 
dingte Sepsis in 5-8 von 1000 Patiententagen vor und ist mit erhöhter Morbidität, Mortalität und medizinischen Kosten assoziiert $[96,102,128,129]$. Im ambulanten Bereich werden katheterassoziierte Komplikationen mit einer Frequenz von 0,29 Zwischenfällen pro Katheterjahr bei Patienten mit tunnelierten Kathetern und mit einer Frequenz von 0,66 Zwischenfällen bei Patienten mit Portsystem beobachtet, wobei in Bezug auf katheterassoziierte Infekte ( 0,17 Zwischenfälle pro Katheterjahr) kein signifikanter Unterschied gezeigt werden konnte [130]. Eine Studie aus den USA untersuchte katheterassoziierte Infekte von 527 Patienten mit HPE und fand, dass es sich bei $80 \%$ der Infekte um einen Blutstrominfekt handelte, bei $17 \%$ um einen Infekt an der Katheteraustrittsstelle und dass weitere $2 \%$ der Infekte im Bereich der Kathetertunnelierung auftraten [131]. Eine Untersuchung aus Italien mit 296 Patienten ergab, dass 76\% der katheterassoziierten Blutstrominfekte durch grampositive Organismen (51\% Staph. epdidermidis, 7\% Staph. aureus), 16\% durch gramnegative Organismen, 3\% durch Pilze und 6\% durch einen polymikrobiellen Infekt ausgelöst wurden [132].

Die Diagnosestellung eines katheterassoziierten Infekts erfolgt zunächst klinisch, bedarf aber der Bestätigung mittels Blutkulturen aus dem ZVK (bei mehrlumigen Kathetern Entnahme von je einem Blutkulturpaar aus jedem Katheterlumen) [133] und aus peripher entnommenem Blut (je eine gesonderte venöse Punktionsstelle, entnommen in einem Abstand von maximal 2 Stunden) $[134,135]$. Bei eindeutigen und ausgeprägten lokalen Infektzeichen (z.B. eitrige Sekretion an der Austrittsstelle) ist der Katheter zu entfernen. Die entfernte Katheterspitze kann prozedurbedingt kontaminiert sein, eine routinemäßige mikrobiologische Untersuchung sollte auf jeden Fall erfolgen.

Eine systemische Antibiotikatherapie sollte durchgeführt und nach Erhalt des Antibiogramms eventuell angepasst werden. Falls sich die Entfernung des Katheters für die HPE oder die sich hieraus ergebenden Konsequenzen als äußerst problematisch darstellen sollten, kann in Ausnahmefällen und bei fehlender vitaler Bedrohung eine Behandlung mit systemischen Antibiotika ohne Entfernung des Katheters versucht werden [96]. Bei tunnelierten ZVKs oder bei Portsystemen kann bei fehlenden lokalen Infektzeichen und bei nur subklinischen Infektzeichen ohne klinische Instabilität eine abwartende Haltung hinsichtlich Katheterwechsel verfolgt werden, es sollte jedoch eine ergänzende antibiotische „Lock-Behandlung“ und eine systemische Antibiotikatherapie eingeleitet werden [136, 137]. In einer Reihe von Studien wurde gezeigt, dass Aminoglykoside oder Penicilline bezüglich Senkung der Kolonisation eine ähnlich gute Wirkung wie Cephalosporine der 3. Generation entfalten $[136,138]$. Gute klinische Erfahrungen wurden mit der Applikation von Vancomycin (3 mL: $2 \mathrm{mg} / \mathrm{mL}$ ) oder von einem Gemisch aus Gentamycin (0,5 $\mathrm{mg} / \mathrm{mL})$ und Vancomycin $(1,0 \mathrm{mg} / \mathrm{mL})$ gemacht $[139,140]$. Falls diese Maßnahmen keine Wirkung zeigen, muss eine chirurgische Entfernung des Katheters bzw. des Portsystems erfolgen. Komplizierte Infekte mit perakuter Symptomatik stellen unabhängig von Blutkulturbefunden Hochrisikokonstellationen dar und erfordern eine schnellstmögliche Entfernung des Katheters kombiniert mit einer systemischen Antibiotikatherapie. Dies gilt ganz besonders bei Sekundärkomplikationen (septische Thrombosen, septische Embolien oder Endokarditis), aber auch für Patienten mit künstlichen Herzklappen [141].

Bei Risikopatienten kann eine prophylaktische „Lock-Therapie“ erwogen werden. In der Studie von Schwartz et al. [142] wurden 126 onkologisch-pädiatrische Patienten mit tunnelierten ZVKs randomisiert. Bei diesen Patienten wurde eine prophylaktische
Lock-Behandlung mit 3 verschiedenen Substanzen verglichen. Die 1. Patientengruppe erhielt Heparin $(10 \mathrm{U} / \mathrm{mL})$, die 2. Gruppe Heparin und Vancomycin $(25 \mu \mathrm{g} / \mathrm{mL})$, und die 3. Gruppe Heparin, Vancomycin und Ciprofloxacin $(2 \mu \mathrm{g} / \mathrm{mL})$. Der Einsatz des Vancomycin- als auch des Vancomycin-Ciprofloxacin-Blocks korrelierte mit einer signifikanten Senkung der katheterassoziierten Infekte, im Vergleich zur Gruppe mit Heparin allein. Bisseling et al. [143] zeigten in einer kontrollierten, aber kleinen Studie mit 30 Patienten, dass bei Kurzdarmpatienten mit einer HPE die katheterassoziierten Infekte mit einer Taurolidine-Lock-Behandlung gegenüber Heparin signifikant gesenkt werden konnten. Eine Antibiotika-Lock-Lösung stellt bei der jetzigen Datenlage kein routinemäßiges Verfahren dar.

\section{Empfehlung 21:}

Die Pflege der Zugangswege bei HEE und HPE sollte durch geschultes Pflegefachpersonal gemäß evidenzbasierten Richtlinien und Pflegestandards durchgeführt werden, um eine hohe Hygienequalität zu erreichen.

[B; starker Konsens]

Um das Risiko für Komplikationen zu reduzieren, sollte ein Pflegestandard implementiert, die Pflegenden geschult und die Katheterpflege dokumentiert werden.

[B; starker Konsens]

Kommentar: Eine Reduktion katheterassoziierter Infektionen kann durch spezifisch geschultes Pflegefachpersonal oder direkte Betreuungspersonen (Schulung zu Indikation, Inspektion, Anlage, Verband und Pflege) und durch Minimierung der Manipulationen am Katheter erreicht werden [88, 101, 144, 145]. Vor Manipulation an der Katheterhalterung oder am Katheter muss eine Desinfektion gemäß hygienischen Pflegestandards durchgeführt werden [96, 101, 146-149].

Aus medizinischen und finanziellen Gründen (Vermeidung von Komplikationen usw.) ist es sinnvoll, geschulte Personen auch für die Handhabung der HEE einzusetzen [150]. Den betreuenden Pflegepersonen zu Hause sollten jederzeit schriftliche Anweisungen zur Verfügung stehen, um eine einheitliche, qualitativ hochstehende Pflege der Zugangswege der künstlichen Ernährung zu garantieren und klar verständliche Anweisungen an die Patienten abzugeben [42, 43]. Möglichst alle an der Pflege beteiligten Personen sollten geschult werden, wodurch notwendige Hygienemaßnahmen besser durchgesetzt werden könnten $[64,151]$. Die Abgabe eines Merkblatts zum richtigen Verhalten im Falle einer sich anbahnenden Infektion (Rötungen, Schwellungen usw.) kann Unsicherheiten bei Patienten und betreuenden Personen ausräumen [146].

\subsection{Applikation}

\section{Empfehlung 22:}

Zur HEE kann bei gastraler Sondenlage und Unverträglichkeit der Bolusernährung bzw. der kontinuierlichen Schwerkrafternährung eine kontinuierliche Pumpenapplikation verwendet werden.

[C; starker Konsens]

Bei jejunaler Sondenlage und bei Verwendung mobiler Systeme sollte die Applikation der Sondenkost kontinuierlich mittels Pumpe erfolgen.

[KKP; starker Konsens] 
Kommentar: Bereits vor über 40 Jahren wurde berichtet, dass bei ausgewählten Patienten mit EE und pumpenassistierter Sondenkostapplikation weniger Komplikationen wie z. B. Diarrhöen beobachtet werden als bei EE über Schwerkraftapplikation [152]. Während die Prävention von Diarrhöen durch Pumpenverwendung möglicherweise günstig beeinflusst wird, vermindert ein Wechsel von Bolusgabe zu kontinuierlicher pumpenassistierter Applikation keine bereits existierende Diarrhö [153]. Die zusammenfassende Beurteilung der aktuellen begrenzten Studienlage lässt den Schluss zu, dass sowohl Bolusapplikationen in den Magen als auch schwerkraftgesteuerte kontinuierliche Applikation möglich sind. Die klinische Erfahrung der Experten spricht für die Anwendung einer Pumpe in ausgewählten Fällen. Deshalb sollte die Applikationsweise individuell in Abhängigkeit von der Grunderkrankung und der Verträglichkeit der Sondenkost angepasst werden.

Im Falle der Nutzung eines mobilen Systems (vgl. Empfehlung 24) ist eine Pumpe notwendig. Die Applikationsrate muss individuell nach Verträglichkeit angepasst werden. Es sollte ein Plan mit den Zielvorgaben hinsichtlich Zusammensetzung, Volumen, Energiezufuhr und Applikationsrate der Sondenkost erstellt werden. Es empfiehlt sich mit geringen Mengen zu beginnen (z.B. $500 \mathrm{~mL}$ über 16 Stunden am ersten Tag) und ein Aufbauschema zu verfolgen. Bei jejunaler Sondenlage ist eine Laufrate von mehr als $150 \mathrm{~mL}$ pro Stunde nicht zu empfehlen, da hier die Verträglichkeit vom Darmlumen und der Transportgeschwindigkeit limitiert wird. Es liegen allerdings keine Studien zu dieser Thematik vor.

\section{Empfehlung 23:}

HPE kann grundsätzlich per Tropfenzähler appliziert werden. Allerdings sollte bei Problemen wie z. B. Blutzuckerschwankungen, langen Laufzeiten und bei der Verwendung mobiler Systeme die Applikation mithilfe automatischer Infusionspumpen erfolgen.

[KKP; Konsens]

Kommentar: Die Zufuhrgeschwindigkeit der PE kann sehr genau über Infusionspumpen oder durch die Schwerkrafteinwirkung und Einstellung der Infusionsgeschwindigkeit über einen Tropfenzähler eingestellt werden. Die Verwendung von Pumpen ist kostenintensiver, aber möglicherweise auch verlässlicher, und die Fließgeschwindigkeit kann einfacher angepasst werden. Dadurch könnte die Verträglichkeit der HPE und die Blutzuckerkontrolle optimiert werden. Klinische Studien, die dies belegen, liegen in deutscher oder englischer Sprache allerdings nicht vor.

\section{Empfehlung 24:}

Mobile Patienten, die die künstliche Ernährung nicht nur über Nacht applizieren (z. B. wegen zu langer Laufzeit oder bei Aspirationsgefahr bei gastraler Ernährung), können mit einem mobilen System (z. B. Rucksack oder Gürteltasche mit Infusionspumpe bzw. enteraler Ernährungspumpe) ausgerüstet werden. [KKP; starker Konsens]

Kommentar: Um die Mobilität bei Patienten mit HEE oder HPE zu fördern bzw. durch die künstliche Ernährung nicht einzuschränken, gibt es mobile Systeme. Für die HPE stehen Rucksäcke zur Verfügung, die den Beutel und die Infusionspumpe beinhalten. Für die HEE stellen wenige Firmen neben Rucksäcken auch Umhänge- und Gürteltaschen zur Verfügung. Diese Systeme bieten sich auch an, wenn das häusliche Umfeld den Einsatz eines Infu- sionsständers unmöglich macht (Teppiche, Treppen usw.). Bei insulinpflichtigen Diabetikern ist der Einsatz einer Infusionspumpe zur Überwachung der Glukosezufuhr sicherer als die Schwerkraftapplikation. Es muss darauf geachtet werden, dass der Patient das System tragen kann, denn durch den Beutel und die Pumpe mit Akku kann im parenteralen Bereich ein Gewicht von über $3 \mathrm{~kg}$ zusammenkommen, im enteralen Bereich gibt es Systeme ab $1 \mathrm{~kg}$. Wichtig ist auch, dass die Produkte zusammenpassen: die Tragesysteme sind für den Gebrauch einer bestimmten Pumpe ausgelegt und können nicht mit jedem beliebigen Beutel bzw. jeder Flasche bestückt werden. Studien zu dieser Thematik liegen nicht vor.

\section{Nahrungsprodukte zur HEE und deren Anwendung \\ $\nabla$}

\section{Empfehlung 25:}

Für die ausschließliche und langfristige HEE über gastrointestinale Sonden und für die orale Ernährung durch Trinknahrung sollen vollständig bilanzierte Trink- bzw. Sondennahrungsprodukte verwendet werden, da diese in ihrer Zusammensetzung den D-A-CH-Empfehlungen entsprechen und den EU-Richtlinien genügen. [A; starker Konsens]

Kommentar: Grundlagen für die Festlegung einer bedarfsgerechten künstlichen Ernährung sind die von der Deutschen Gesellschaft für Ernährung, der Österreichischen Gesellschaft für Ernährung, der Schweizerischen Gesellschaft für Ernährungsforschung und der Schweizerischen Vereinigung für Ernährung (D-A-CH) veröffentlichten „Referenzwerte für die Nährstoffzufuhr“ [154]. Diese Referenzwerte liegen auch dem Begriff „vollständig bilanziert“ zugrunde, entsprechend der Diätverordnung aus Deutschland [155] sowie der zur Überarbeitung anstehenden EU-Richtlinie (2009/39/EU). Dieser Begriff besagt, dass eine vollständige Bedarfsdeckung erreicht wird, wenn man sich ausschließlich vom vollständig bilanzierten Produkt in der empfohlenen Menge ernährt und keinen von der Norm abweichenden Bedarf hat. Mit einer vollständig bilanzierten künstlichen Ernährung werden demnach alle lebensnotwendigen Makro- und Mikronährstoffe (Aminosäuren, Fettsäuren, Vitamine, Mengenund Spurenelemente), entsprechend dem für die gesunde Bevölkerung berechneten Bedarf zugeführt. Ergänzende bilanzierte Diäten sind Trink- und Sondennahrungsprodukte, die in einer definierten Tagesverzehrsmenge vorgegebene Höchstmengen von Mineralstoffen und Vitaminen nicht überschreiten dürfen. Sie enthalten entweder nicht alle Makro- und Mikronährstoffe oder enthalten diese in einem Verhältnis, das sie als ausschließliche Ernährung ungeeignet macht. Die definierte und bilanzierte $\mathrm{Zu}-$ sammensetzung von industriellen Produkten ermöglicht eine einfache Festlegung der Dosierung (Angabe der Energiedichte, der Eiweiß- und Fettkonzentration und -art sowie der Konzentrationen an Mikronährstoffen).

Selbst zubereitete Ernährungsprodukte bzw. nicht verordnungsfähige Sondennahrungsprodukte sollen für die HEE nicht verwendet werden, weil sie den Anforderungen an eine von den Nährstoffen her ausgewogene, hygienisch unbedenkliche und bezüglich der Viskosität optimale Nährstoffzufuhr kaum gerecht werden. Der zusätzliche Zeitaufwand bei der Eigenherstellung von Sondenkost ist bei qualitativ schlechterer und langfristig nicht konstanter Versorgung des Patienten sowie der erhöhten Verstopfungsgefahr der Sonde nicht gerechtfertigt [156-161]. 
Als orale Trinknahrung, auch ONS (oral nutritional supplements) genannt, werden in Deutschland laut Diätverordnung nur bilanzierte Trinknahrungsprodukte zugelassen [155]. Aufgrund des Gesetzescharakters der Empfehlung wurde der Evidenzgrad A festgelegt.

Der Nährstoffbedarf kann bei verschiedenen Erkrankungen aufgrund pathophysiologischer und pathobiochemischer Vorgänge gegenüber diesen Referenzwerten verändert sein. Dies kann bei einzelnen Krankheitsbildern eine individuelle Anpassung einzelner Nährstoffe erfordern.

\section{Empfehlung 26:}

Bei der Auswahl des Produkts zur HEE sollten folgende Aspekte berücksichtigt werden: 1. die gastrointestinale Funktion, 2. der Volumenbedarf, 3. die Sondenlage sowie 4. krankheitsspezifische Aspekte.

[KKP; starker Konsens]

Patienten mit intaktem Gastrointestinaltrakt sollten in der Regel mit iso- oder hochkalorischen hochmolekularen Standarddiäten versorgt werden.

[KKP; starker Konsens]

Sofern keine Kontraindikationen bestehen, sollten Produkte mit Ballaststoffen/Nahrungsfasern verabreicht werden.

[B; starker Konsens]

Kommentar: Bezüglich der Art der Makronährstoffe unterscheidet man [162]:

- Hochmolekulare oder makromolekulare Sondenkost (früher „nährstoffdefinierte Diät“"), die als Makronährstoffe 15-20\% Proteine (Milch- oder Sojaprotein ), 25 - 30\% langkettige Triglyzeride (Pflanzenöle) und 50-60\% Kohlenhydrate (Oligo-, Polysaccharide) enthalten und entweder mit oder ohne Ballaststoffe angeboten werden.

- Niedermolekulare oder mikromolekulare Sondenkost (früher „chemisch definierte Diät“), in denen die Makronährstoffe in niedermolekularer Form vorliegen: Proteine als Peptide und Aminosäuren, Fette teilweise als MCT-Fette, die unabhängig von Pankreaslipasen absorbiert werden können sowie carnitinunabhängig in Mitochondrien oxidiert werden und Kohlenhydrate als Di- und Oligosaccharide. Diese Produkte werden bei eingeschränkter Digestions- und Absorptionsfähigkeit im oberen Gastrointestinaltrakt besser resorbiert.

Siehe hierzu auch Valentini et al., DGEM-Terminologie in der Klinischen Ernährung [13].

Als Standardsondenkost sollte eine hochmolekulare Sondenkost mit Ballaststoffen verwendet werden (vgl. Empfehlung 27). Proteinreiche Produkte werden bei (drohender) Sarkopenie bevorzugt. Energiereiche Produkte $(>1 \mathrm{kcal} / \mathrm{mL}$ ) sind sinnvoll, wenn eine Flüssigkeitsrestriktion berücksichtigt werden muss. Bei jejunaler Lage der Ernährungssonde sollten Produkte mit hoher Osmolarität gemieden werden. Bei individuellen Intoleranzen oder speziellen Erkrankungen sind weitere Aspekte, z.B. spezieller Elektrolyt- oder Proteinbedarf bei renalen Erkrankungen, teilweiser Ersatz von LCT durch MCT bei Malassimilation oder hepatobiliären Erkrankungen, zu beachten (vgl. jeweils organspezifische Leitlinien).

Standardsondenkost gibt es grundsätzlich als niedrigkalorische $(<0,9 \mathrm{kcal} / \mathrm{mL})$, isokalorische $(0,9-1,2 \mathrm{kcal} / \mathrm{mL})$ oder hochkalorische (>1,2 kcal/mL) Ernährung. Die iso-und hochkalorischen Sondennahrungsprodukte sind für die heimenterale Ernährung verordnungsfähig, während die niedrigkalorischen im Homecare-
Bereich keine Rolle spielen, weil sie nicht verordnungsfähig sind. Die Verordnungsfähigkeit der enteralen Ernährung ist in der am 1.10.2005 in Kraft getretenen Bekanntmachung des Bundesministeriums für Gesundheit und soziale Sicherung im Bundesanzeiger geregelt [163].

Fast alle Sondennahrungsprodukte sind gluten- und nahezu laktosefrei, sie enthalten weder Purin noch Cholesterin. Bei erhöhtem Proteinbedarf stehen Produkte mit einem erhöhten Eiweißanteil (>20\%) zur Verfügung. Bei bestehenden Allergien gegen bestimmte Nahrungsproteine (z.B. Milcheiweiß, Sojaeiweiß) kann auf Produkte ausgewichen werden, die die jeweiligen Proteine nicht enthalten. Der Tagesbedarf an Elektrolyten, Vitaminen und Spurenelementen wird bei einer täglichen Zufuhr von ca. 1500 kcal erreicht. Der Wassergehalt der Trink- und Sondennahrungsprodukte liegt zwischen 70\% (2 kcal $/ \mathrm{mL}$ ) und $85 \%$ ( $1 \mathrm{kcal} / \mathrm{mL}$ ). Dies sollte bei der Berechnung der separat zu verabreichenden Flüssigkeitsmenge einkalkuliert werden.

Die Empfehlung zu den Ballaststoffen/Nahrungsfasern begründet sich aus den allgemeinen Ernährungsempfehlungen der DGE. Der Ballaststoff-/Nahrungsfaseranteil der gängigen Sondenkostprodukte liegt zwischen 5 und 15\%. Es stehen Produkte mit einem Gemisch an löslichen (weitgehend fermentierbaren) und unlöslichen (kaum fermentierbaren) Ballaststoffen/Nahrungsfasern und Produkte, die lediglich lösliche Ballaststoffe/Nahrungsfasern enthalten, zur Verfügung. Ballaststoffe/Nahrungsfasern können die intestinale Mikrobiota positiv beeinflussen (Präbiotika) und sowohl bei Diarrhö als auch bei Obstipation wirksam sein [164]. Ein Gemisch aus löslichen und unlöslichen Ballaststoffen/Nahrungsfasern ist hinsichtlich der gastralen Motilität von Vorteil gegenüber unlöslichen Ballaststoffen/Nahrungsfasern allein [165]. Unlösliche Faserstoffe können bei ungenügender Flüssigkeitszufuhr vermehrt Obstipation verursachen (ungenügende Quellung). Die Empfehlung für Ballaststoffe/Nahrungsfasern gilt wahrscheinlich auch für Kinder [166].

\section{Empfehlung 27:}

Bei Patienten mit erkrankungsbedingter Einschränkung der Digestions- oder Absorptionsfähigkeit kann bei Unverträglichkeit von Standardnahrung eine niedermolekulare Sondenkost verwendet werden. Bei hepatischer Enzephalopathie oder hochgradig eingeschränkter Nierenfunktion sollte eine speziell adaptierte Sondennahrung zur HEE erwogen werden.

[KKP; starker Konsens]

Kommentar: Beispiele für den sinnvollen Einsatz von speziell adaptierter Sondennahrung im Rahmen der HEE sind die Verwendung von niedermolekularer Trink- oder Sondennahrung bei gastroenterologischen Patienten mit Einschränkung der Digestions- oder Absorptionsfähigkeit, um die Nährstoffaufnahme zu verbessern, von eiweißreicher, elektrolyt- und volumenreduzierter Trink- oder Sondennahrung bei Patienten mit dialysepflichtiger Niereninsuffizienz, oder von Sondennahrung mit einem höheren Anteil an verzweigtkettigen Aminosäuren bei Patienten mit hepatischer Enzephalopathie und Ammoniakerhöhung. Weitere Erläuterungen und Darstellung der Literatur siehe entsprechende DGEM-Leitlinien zu Krankheitsbildern. 


\section{Empfehlung 28:}

Bei Indikation zur künstlichen Ernährung und einem Energiedefizit von $500-800 \mathrm{kcal}$ pro Tag sollte primär supplementäre orale Trinknahrung (ONS) verwendet werden.

[B; starker Konsens]

Kommentar: ONS ist zur langfristigen Behandlung von Malnutrition insbesondere älterer Menschen geeignet [167]. Allerdings zeigt die Praxis, dass längerfristig maximal $500 \mathrm{~mL}$ pro Tag mittels oraler Trinknahrung aufgenommen werden, weshalb diese Menge in zahlreichen Studien eingesetzt wurde [167, 168]. Diese Menge entspricht je nach Energiedichte des Produkts einer Energiezufuhr von etwa $500-800 \mathrm{kcal}$. Einzelheiten zur Indikation und Verwendung von oraler Trinknahrung wurden kürzlich in einem Algorithmus zusammengestellt, der Teil der DGEM-Leitlinie ist und einem analogen Abstimmungsprozess unterzogen wurde [169]. Die B-Empfehlung basiert stellvertretend auf den Arbeiten von Beck et al. [167] und Botella-Carretero et al. [168], wobei dort in erster Linie die Effektivität der Trinknahrung untersucht wurde und nicht die Menge im Sinn einer Dosisfindungsstudie.

\section{Empfehlung 29:}

Enterale Ernährungsprodukte können nach Öffnung bei Raumtemperatur innerhalb von 8 Stunden, bei Aufbewahrung im Kühlschrank innerhalb von 24 Stunden verwendet werden und während der Applikation bis zu 24 Stunden am Infusionsständer ohne Kühllagerung verbleiben.

[C; starker Konsens]

Kommentar: Bei der Verwendung industriell hergestellter enteraler Ernährungsprodukte sind die Angaben und Nutzungshinweise des Herstellers einzuhalten. Insbesondere ist darauf $\mathrm{zu}$ achten, dass die Lagerung und Verabreichung von enteralen Ernährungsprodukten bei Raumtemperatur $\left(15-25^{\circ} \mathrm{C}\right)$ unter Beachtung des Mindesthaltbarkeitsdatums erfolgt und die Aufbewahrung in praller Sonne oder in Heizungsnähe vermieden wird. Durch Lagerung bei höheren Temperaturen (bis zu $40^{\circ} \mathrm{C}$ ) riskiert man schnellere, ausgeprägtere Aufrahmung, verstärktes Absinken des pH-Werts, Zunahme der Auslaufzeit, Verschlechterung der Sondengängigkeit, Dunkelverfärbung des Produkts sowie beschleunigten Vitaminabbau. Andauernde relative Luftfeuchte über $70 \%$ sollte vermieden werden, um ein Knicken der Kartonkanten bei gestapelter Palettenware zu vermeiden $[44,58]$. Die verkürzte Verwendungsdauer nach Anbruch ist primär aus hygienischer Sicht gegeben (potenzielle mikrobielle Kontamination). Basierend auf klinischer Erfahrung sollten zur HEE geschlossene Systeme, bei denen das Überleitungssystem direkt an den Ernährungsbeutel angeschweißt ist, bevorzugt verwendet werden. Bei industriell hergestellten Produkten muss der Hersteller die Vorgaben des Lebensmittelrechts einhalten (z.B. Lebensmittel-Kennzeichnungs-Verordnung, Fertigpackungs-Verordnung, Diät-Verordnung u.v.m.). Daher sind diese Vorgaben zu beachten und vom Anwender einzuhalten. Ausführliche Unterlagen zu den einzelnen Produkten sind in Firmenbroschüren und über die Angaben der Firmen im Internet zu erhalten.

\section{Empfehlung 30:}

Enterale Ernährungsprodukte sollten zimmerwarm verabreicht werden. Bei pumpengesteuerter Nahrungszufuhr mit niedriger Laufrate (bis $70 \mathrm{~mL} / \mathrm{h}$ ) kann auch kühlschrankkalte Sondenkost verwendet werden, da diese sich während der Infusion im Überleitsystem an die Raumtemperatur anpasst. Bei Nahrungspausen von mehr als 4 Stunden kann das gesamte System (inklusive Überleitgerät) in den Kühlschrank gestellt werden.

[KKP; starker Konsens]

Kommentar: Diese Empfehlungen sind klinischer Konsens, Primärliteratur existiert dazu nicht. In Ausnahmefällen ist ein schonendes langsames Erwärmen der enteralen Ernährungsprodukte auf max. $40^{\circ} \mathrm{C}$ möglich. Gekühlte Nahrung (z. B. bei angebrochenen Behältern) soll ca. 2 Stunden vor Applikationsbeginn aus dem Kühlschrank genommen werden. Das Erwärmen kann im Wasserbad oder, wie vom Robert Koch-Institut (RKI) [170] empfohlen, mittels Mikrowellengerät erfolgen.

\section{Nahrungsprodukte zur HPE und deren Anwendung}

\section{Empfehlung 31:}

Zur HPE sollten „All-in-One-Nährmischungen“ verwendet werden, denn sie sind als sichere, effektive und risikoreduzierende Standards zur Durchführung der HPE etabliert.

[KKP; starker Konsens]

Mehrflaschensysteme sollten wegen höherer Risiken und aufwendigerer Handhabung durch das Personal und den Patienten nicht verwendet werden.

[B; starker Konsens]

Kommentar: „All-in-One“ (AiO)-Nährmischungen für die HPE sind industriell oder individuell hergestellte Ernährungsbeutel für die PE, die alle Komponenten der HPE enthalten und sich nicht nur in vielen Bereichen der Klinik, sondern auch in der ambulanten Versorgung durchgesetzt haben. Bei der Durchführung einer HPE zeigt die Verwendung von AiO-Nährmischungen mikrobiologische, metabolische, kompatibilitäts- und handhabungsmäßige Vorteile gegenüber anderen Systemen, welche auf Einzelkomponenten bzw. Kombinationslösungen basieren [171-174]. In der Versorgungspraxis ist die Bereitstellung der AiO-Nährmischungen auf 2 Arten möglich:

1. Industriell gefertigte Zwei- oder Dreikammerbeutel (Mehrkammerbeutelkonzept, d.h. stabile und sterile MakronährstoffKomponenten in voneinander abgetrennten Kammern [175]), die vorgegebenen Rezepturen folgen, allerdings in vielen Varianten angeboten werden und i.d.R. mit Mikronährstoffen supplementiert werden müssen. Beim Zweikammerbeutel, der eine Kammer mit Glukoselösung und eine Kammer mit Aminosäurelösung und Elektrolyten enthält, wird kurz vor der Anwendung am Patienten eine Fettemulsion mittels Transferset zugegeben und damit ein AiO-Makronährstoffgemisch generiert. Dreikammerbeutel enthalten alle Makronährstoffe und eine Basismenge an Elektrolyten in 3 abgeteilten Kompartimenten, wobei $2 \mathrm{Kam}-$ mern analog zum Zweikammerbeutel gefüllt sind und die dritte Kammer eine Fettemulsion enthält. Die Substrate werden unmittelbar vor der intravenösen Applikation gemischt, indem man die Trennvorrichtungen/Nähte zwischen den Beutelkammern löst. Vitamine und Spurenelemente werden vor der unmittelbaren 
Anwendung in die Mischung respektive die entsprechende Kammer (z.B. Spurenelemente zu Aminosäuren) zugespritzt oder separat intravenös infundiert (siehe Empfehlung 38). Die Haltbarkeit der Zwei- bzw. Dreikammerbeutel beträgt bei Raumtemperatur mindestens 12 Monate, solange die Nähte intakt und keine Zusätze zugespritzt wurden. Nach Mischung und Zugabe von Mikronährstoffen ist die Haltbarkeit i.d. R. auf $24 \mathrm{~h}$ beschränkt, wobei das jeweilige Haltbarkeitsdatum des Herstellers sowie weitere Anwendungsregeln wie Verwendung eines Lichtschutzbeutels etc. beachtet werden müssen.

2. Individuelle AiO-Nährmischungen (Compounding), die nach Vorgaben eines Arztes in speziell ausgestatteten Apotheken und Herstellungsbetrieben unter aseptischen Bedingungen und Beachtung der Kompatibilitäten gemischt werden. Sie erlauben die Herstellung von patientenspezifischen, am individuellen Bedarf orientierten Mischungen (energie-, volumen- und substratadaptiert) von Makro- und Mikronährstoffen. Im Gegensatz zu den industriell gefertigten Zwei- oder Dreikammerbeuteln können beim Compounding bereits bei Herstellung Vitamine und Spurenelemente zugemischt werden, sofern die Herstellung am Tag der Anwendung erfolgt. Vorgaben zur Stabilität, Kompatibilität und Dokumentation sind bei der Zubereitung nötig und erfordern im Einzelfall detaillierte und produktspezifische Laboruntersuchungen. Extrapolation von Daten sind in diesen äußerst komplexen und metastabilen Gemischen in der Regel schwierig oder unmöglich, es können jedoch erhebliche Unterschiede, z.B. in der Fettstabilität, unter Zusatz von Spurenelementen auftreten. Diese Beutel werden wegen der limitierten Stabilität in der Regel am Tag der Anwendung oder für die Verwendung innerhalb von maximal 7 Tagen einmal wöchentlich gemäß den Stabilitätsdaten hergestellt. Ihr Transport und die mehrtägige Aufbewahrung erfordert Kühllagerung bei $2-8^{\circ} \mathrm{C}$.

\section{Empfehlung 32:}

In der Regel können für die HPE Standardlösungen verwendet werden. Bei einigen Krankheitsbildern können individuell zusammengesetzte Nährlösungen erforderlich sein. [C; starker Konsens]

Kommentar: Die Verwendung von standardisierten parenteralen Nährmischungen vereinfacht die Verschreibung, deren Herstellung und reduziert Komplikationen; sie verbessert die Patientensicherheit und die Behandlungseffizienz [176-178].

Patienten, bei denen aufgrund ihrer Erkrankung industriell vorproduzierte Standardprodukte mit festgelegten Rezepturen (z. B. Dreikammerbeutel) den jeweiligen Nährstoff- und/oder Volumenbedarf nicht decken können, müssen mit individuell berechneten Infusionsregimen versorgt werden, z. B. Kurzdarmpatienten mit hohen intestinalen Verlusten, schwer mangelernährte Patienten mit erhöhtem absoluten und relativen Bedarf an einzelnen Makro- und Mikronährstoffen, dialysepflichtige Patienten mit erhöhtem Eiweißbedarf und Volumenbeschränkung, Patienten mit Leberfunktionseinschränkungen, bei denen die Verwendung spezieller Eiweißmischungen mit vermehrt verzweigtkettigen Aminosäuren indiziert ist. Hier bietet sich das „Compounding“ an, sofern es verfügbar ist.

Eine PE, bei der die Makrosubstrate durch Einzelkomponenten („Mehrflaschensystem“) verabreicht werden, ist aus folgenden Gründen für die häusliche Versorgung nicht geeignet, auch wenn bei ausschließlicher Betrachtung der Kosten für die Makro- nährstoffe die Versorgung mit Einzelkomponenten rein rechnerisch günstiger erscheinen kann:

- Der Zeitaufwand und damit die Personalkosten für das Anschließen der einzelnen Substrate sind deutlich höher.

- Durch die zusätzlich notwendigen Infusionsleitungen, Konnektoren und Verschlussmechanismen ist das Risiko für das Auftreten von Infusionsleitungsproblemen deutlich erhöht.

- Die Anwendung des Einzelkomponentensystems führt zu einer erheblichen Einschränkung der Mobilität des Patienten und wegen der zahlreichen Infusionsleitungen zu einer Gefährdung der Patientensicherheit in der häuslichen Umgebung.

- Für die optimale Nutzung der Nährstoffe sind diese zeitgleich zuzuführen, insbesondere müssen gleichzeitig mit den Aminosäuren auch Nichteiweißkalorien zugeführt werden, damit der anabole Effekt der Proteinzufuhr zum Tragen kommt. Dies wäre im Einzelkomponentenmodell nur durch Einsatz mehrerer Pumpen zu erreichen, was im häuslichen Umfeld nicht praktikabel und außerdem sehr kostenintensiv ist. Die nicht koordinierte Zufuhr der Einzelsubstrate kann zu Inkompatibilitäten mit Ausfällungen führen und das Risiko für das Auftreten metabolischer Komplikationen (Hyperglykämie, Hypertriglyzeridämie) erhöhen.

- In mehreren Studien konnte nachgewiesen werden, dass das Infektionsrisiko mit der Zahl der Manipulationen am zentralen Zugang steigt, eine unnötige Erhöhung des potenziell lebensbedrohlichen Kathetersepsisrisikos durch Verwendung eines Mehrflaschenkonzepts ist ethisch nicht vertretbar; zudem führt die Therapie der Kathetersepsis zu einer erheblichen Steigerung der Gesamtkosten [101, 145, 147, 179-181].

\section{Besonderheiten}

Die auch für die periphere Applikation geeigneten Dreikammerbeutel zeichnen sich durch eine niedrigere Osmolarität, einen im Verhältnis zum Energiegehalt erhöhten Volumen- und Fettanteil sowie einen niedrigeren Glukose- und Aminosäurenanteil aus. Dadurch können diese Beutel für Patienten in diabetischer Stoffwechsellage und/oder bei erhöhtem Volumenbedarf z. B. bei gastrointestinalen Volumenverlusten besonders geeignet sein. Wegen der inflammatorisch veränderten Stoffwechsellage, die u.a. durch eine Insulinresistenz und erhöhte Fettoxidation gekennzeichnet ist, sind diese Nährlösungen auch für Patienten mit Tumorkachexie besonders geeignet.

Eine besondere Herausforderung stellen HPE-Mischungen für pädiatrische Patienten dar, da neben der Deckung des aktuellen Energie- und Eiweißbedarfs infolge der Aktivität und dem basalen Metabolismus auch der Bedarf für das Wachstum eingerechnet werden muss, was z.B. auch die Stabilität einer AiO-Nährmischung stark einschränken und zur Verwendung von binären Lösungen (Glukose und Aminosäuren) mit Zusätzen und separater Fettzufuhr führen kann. In der Kinder-HPE hat sich die Verwendung von n-3-Fettsäuren zur Verhinderung hepatobiliärer Komplikationen etabliert.

\section{Empfehlung 33:}

Im Einzelfall können in der HPE spezielle pharmakologisch wirksame Zusätze oder Lösungen (z. B. Glutamin, n-3-Fettsäuren)² verwendet werden.

[C; starker Konsens]

\footnotetext{
${ }^{2}$ Arzneimittel sind hiervon ausgenommen.
} 
Kommentar: Die Verwendung von Zusätzen bzw. mit funktionellen Substraten angereicherten Lösungen, d.h. Pharmakonutrition, ist inzwischen auch im HPE-Bereich zunehmend akzeptiert, nachdem mehrere Studien aus den letzten Jahren zeigten, dass solche Supplemente gut vertragen werden [182-186]. Allerdings zeigten diese Studien für Glutamin keinen eindeutigen bzw. allenfalls marginalen Benefit [182 - 184]. Für n-3-Fettsäuren konnten Vorteile hinsichtlich Prävention von PE-assoziierter Fettlebererkrankung (PNALD) u.a. metabolischer Veränderungen bei Erwachsenen $[185,187]$ und Kindern $[186,188]$ in kontrollierten Pilotstudien gezeigt werden.

In mehreren Studien konnte gezeigt werden, dass Fischölemulsionen einerseits einen protektiven Effekt auf die Leberfunktion und andererseits eine schnellere Erholung der durch herkömmliche Sojaölemulsionen bedingten erhöhten Leberparameter bewirken [189-193]. Dies stellt den langfristigen Einsatz reiner Sojaölemulsionen ohne solche Zusätze infrage.

\section{Empfehlung 34:}

Die Glukosezufuhr unter HPE sollte beim Erwachsenen 2-4g/kg Körpergewicht/Tag betragen.

[KKP; starker Konsens]

Eine höherere Zufuhr als $4 \mathrm{~g} / \mathrm{kg}$ Körpergewicht/Tag kann beim

Erwachsenen nicht empfohlen werden, da ein erhöhtes Komplikationsrisiko, z. B. hinsichtlich Infektionsrate oder Leberschädigung besteht.

[C; starker Konsens]

In einer Reihe von klinischen Studien konnte gezeigt werden, dass es unter HPE vermehrt zu Steatose, Steatohepatitis, intrahepatischer Cholestase, Cholezystolithiasis, Cholezystitis kommen kann [194-196]. Hepatische Komplikationen sind mit 15-40\% relativ häufig [197]. Solche Komplikationen manifestieren sich meist erst nach Monaten oder Jahren. Meist handelt es sich um eine sonografisch festgestellte Fettleber, sehr viel seltener um eine intrahepatische Cholestase oder Steatohepatitis, die zu einer Zirrhose oder zu einer terminalen Leberkrankheit führen können. Eine chronische Cholestase wird beschrieben als eine Erhöhung der Leberparameter (>1,5-mal der oberen Normwerte von alkalischer Phosphatase, $\gamma$-Glutamyltransferase und konjugiertem Bilirubin) über mindestens 6 Monate [191]. Die Ätiologie der hepatischen Komplikationen ist unklar, kann aber zumindest teilweise durch zu hohe Glukosezufuhr bedingt sein. Verschiedene andere Faktoren wie selektiver Mangel an bestimmten Aminosäuren, hormonelle Veränderungen, Substratüberschuss, reduzierte Motilität der Gallenblase, Störung des enterohepatischen Kreislaufs und möglicherweise Dysbiose des Dünndarms mit portaler Endotoxinämie werden diskutiert [7, 191, 195, 196]. Potenziell hepatotoxische Substanzen bzw. Medikamente sollten so weit wie möglich vermieden werden. Von ernährungsmedizinischer Seite sind die wichtigsten Maßnahmen, um einer PE-induzierten Hepatopathie vorzubeugen und diese zu behandeln, die Verabreichung eines Nährstoffgemischs (Glukose/Fett) statt Glukose isoliert, das Vermeiden eines Overfeeding (>35 kcal $/ \mathrm{kg}$ $\mathrm{KG} / \mathrm{Tag}$ ) und die Gabe von großen Glukosemengen (>5 g/kg KG) Tag) [195]. Nach Erfahrung von Experten sollte bei Langzeit-HPE bereits eine Glukosemenge von $4 \mathrm{~g} / \mathrm{kg} \mathrm{KG} / \mathrm{Tag}$ möglichst nicht überschritten werden. Die Glukosezufuhr bei zyklischer HPE muss der metabolischen Toleranz angepasst werden und ist meist entscheidend für die notwendige Dauer der HPE.

\section{Empfehlung 35:}

Eine zumindest minimale enterale Nahrungszufuhr (10-20 mL Substrat/h) sollte parallel zur HPE durchgeführt werden, um das Risiko von Komplikationen unter HPE zu reduzieren.

[B; starker Konsens]

Bei Cholestase unter HPE kann mit Ursodeoxycholsäure behandelt werden.

[C; starker Konsens]

Kommentar: Als Therapie der Cholestase unter HPE wurde neben Beschränkung der Glukosezufuhr der Effekt von paralleler oraler oder enteraler Nahrungszufuhr [198, 199] und Ursodeoxycholsäure $[200,201]$ in klinischen Studien untersucht und für wirksam befunden. Trotz des Evidenzgrads der Literatur von III-IV wurde für die zumindest minimale EE die Empfehlungsstärke B gewählt, weil sie außer Reduktion von hepatischen Komplikationen wie Cholestase auch Reduktion von Infektionskomplikationen bewirkt. Auch Infektbehandlung, z.B. mit Metronidazol und zyklische statt kontinuierliche Applikation der HPE, könnte wirksam sein [199].

Die Konkrementbildung wird sowohl durch eine Verlangsamung der intestinalen Peristaltik mit konsekutiver Cholestase als auch durch erniedrigte Cholezystokininkonzentrationen im Pfortadersystem begünstigt. Experimentell wurden auch Cholezystokinin $[202,203]$ sowie eine schnelle Infusion von Aminosäurelösungen [204] eingesetzt. In der klinischen Routine wurden diese Ansätze allerdings bisher nicht erprobt und erscheinen auch wenig praktikabel.

\section{Empfehlung 36:}

Zur Prophylaxe und Therapie einer Osteomalazie und Osteoporose unter HPE kann eine optimierte Kalzium-, Phosphat- und Vitamin-DZufuhr empfohlen werden.

[C; Konsens]

Bisphosphonate sollten zur Therapie einer verminderten Knochendichte unter HPE eingesetzt werden.

[B; Konsens]

Kommentar: Patienten mit HPE haben ein höheres Risiko für Verlust an Knochendichte. Die Prävalenz der Osteoporose bei Patienten mit HPE liegt über 40\% [197, 205 - 207]. Die Pathogenese der Osteomalazie und Osteoporose unter PE ist weitgehend unklar; diese Erkrankungen manifestieren sich meist erst nach Monaten oder Jahren. Die Knochenveränderungen sind vermutlich Ausdruck einer nicht optimalen Kalzium-, Phosphat- und/oder Vitamin-D-Zufuhr, einer fehlenden körperlichen Belastung, einer fehlenden Lichtexposition oder Folge von Medikamenten wie Heparin oder Steroiden [208]. Therapeutische Maßnahmen zielen auf eine bedarfsgerechte Substratzufuhr sowie auf die Vermeidung anderer Risiken ab.

Bei Patienten, die auf HPE nicht verzichten können und bereits an Osteoporose leiden, wird die intravenöse Gabe von Bisphosphonaten (z.B. Pamidronat: $30 \mathrm{mg}$ alle 3 Monate; Ibandronat: $3 \mathrm{mg}$ alle 3 Monate; Clodronat: $1500 \mathrm{mg}$ alle 3 Monate; Zoledronat: $5 \mathrm{mg}$ einmal jährlich) oder die subkutane Gabe von humanen monoklonalen Antikörpern (Denosumab: $60 \mathrm{mg}$ alle 6 Monate) empfohlen [209-213]. In kleinen Studien konnte gezeigt werden, dass sich durch Pamidronat die Knochendichte bei HPE-Patienten verbessern lässt $[214,215]$. Eine doppelblinde randomisierte und placebokontrollierte Studie zeigte, dass unter HPE die 
Therapie mit Clodronat einen signifikanten positiven Effekt auf die Knochenresorption hat [210].

In alten Therapiekonzepten ist der Vitamin-D-Substitution wenig Beachtung geschenkt worden. Moderne Konzepte hingegen empfehlen die adäquate Vitamin-D-Substitution unter HPE mit dem Ziel, einen optimalen Serumspiegel zu erreichen $(75-100 \mathrm{nmol} /$ L; 30-40 ng/mL) [216]. Damit kann eine vorhandene Osteomalazie korrigiert und die Behandlung einer Osteoporose optimiert werden [217]. Obwohl die herkömmlichen intravenösen Multivitaminpräparate i.d.R. die übliche Tagesdosis des Vitamin D enthalten, ist eine zusätzliche Gabe von reinen Vitamin-D-Präparaten in vielen Fällen von Patienten unter HPE notwendig [218]. Zu beachten ist, dass die DGE 2012 für Vitamin D neue Referenzwerte herausgebracht hat und die Empfehlungen für Vitamin D angehoben wurden (von 5 auf $20 \mu \mathrm{g} / \mathrm{d}$ beim Erwachsenen) [154].

\section{Empfehlung 37:}

Individuelle AiO-Nährmischungen (Compounding) sollen bei $2-8^{\circ} \mathrm{C}$ gelagert werden, industriell gefertigte Mehrkammerbeutel sind gemäß Herstellerangaben zu lagern. Generell sollen AiO-Nährmischungen bei Raumtemperatur verwendet und innerhalb von 24 Stunden infundiert werden.

[A; starker Konsens]

Infusionssysteme (Lipidinfusionen oder individuell zubereitete AiOPräparate), die für HPE genutzt werden, sollten alle 24 Stunden gewechselt werden.

[B; starker Konsens]

Kommentar: Die Empfehlungen der Kommission für Krankenhaushygiene und Infektionsprävention des Robert Koch-Instituts (RKI) sollen bei der Durchführung der HPE Anwendung finden. Fehlerhafte Hygienemaßnahmen, d.h. die Nichtbeachtung der Prinzipien der Asepsis bei der Zubereitung von Medikamenten und ihrer Applikation durch Injektion oder Infusion, haben in der Vergangenheit wiederholt zu bakteriellen oder viralen Infektionen geführt. Besonders beachtet werden sollten die Empfehlungen zur Händehygiene und zur Prävention von gefäßkatheterassoziierten Infektionen sowie die Empfehlungen „Anforderungen an die Hygiene bei Punktionen und Injektionen“ [101, 219, 220].

Besonders aus Hygienegründen, d. h. zur Vermeidung von Mikroorganismenwachstum, sind AiO-Nährmischungen kühl bei $2-8^{\circ}$ C zu lagern und es ist auf eine lückenlose Kühlkette zu achten. Die Dauer der Kühlaufbewahrung muss auf der Beschriftung hinterlegt und dokumentiert sein. Die anschließende Applikationsdauer von 24 Stunden muss ebenfalls dokumentiert sein. Eine Anwendung für HPE kann zyklisch (über Nacht bis 14 Stunden) oder kontinuierlich (über 24 Stunden) erfolgen. Bei beiden Bereitstellungsweisen ist eine strikte aseptische Arbeitsweise erforderlich, da eine Endsterilisation einer infusionsfertigen AiOMischung nach Komplettzubereitung nicht möglich ist [221].

Die A-Empfehlung basiert auf dem regulatorischen bzw. legalen Charakter der Empfehlung. Der zweite Satz ist daraus abgeleitet und deshalb auf eine B-Empfehlung abgestuft.

\section{Empfehlung 38:}

Spurenelemente und/oder Vitamin-Kombinationspräparate sind integraler Bestandteil einer parenteralen Ernährung und können zu AiO-Nährmischungen zugespritzt werden, wenn aspetische Zugabe, Kompatibilität sowie Stabilität gewährleistet und dokumentiert sind. [C; starker Konsens]
Kommentar: AiO-Nährmischungen für die HPE sind für den unmittelbaren Gebrauch vorbereitet, enthalten aber keine Vitamine oder Spurenelemente. Adaptionen (Zumischungen) sind unter aseptischen Bedingungen (Laminar-Airflow) und nach pharmazeutischen Vorschriften vorzunehmen. Diese Adaptionen sollten von Fachpersonal durchgeführt werden [222 - 225]. Aus strukturellen und/oder organisatorischen Gründen kann das Zusetzen von Mikronährstoffen im Einzelfall nach pharmazeutischer, dokumentierter Anweisung unmittelbar vor der Anwendung und patientennah durch speziell geschulte Personen (meist „Offlabel“-Anwendung) durchgeführt werden. Die Arbeitsschritte zur patientennahen Vorbereitung der AiO-Nährmischung unmittelbar vor der Anwendung sollen in Standardarbeitsanweisungen hinterlegt und dokumentiert sein [219].

Aus physikochemischen Gründen ist die gleichzeitige Verabreichung von Spurenelementen und Multivitaminprodukten in AiO-Nährmischungen kritisch, denn es kann zu einem verstärkten Abbau oxidationsempfindlicher Vitamine oder zu einer verstärkten (Lipid-)Peroxidation kommen. Oxidations-/Reduktionsreaktionen können durch Licht (insbesondere UV-Strahlung) weiter verstärkt werden $[122,226]$. Die kombinierte Verabreichung von Multivitaminprodukten und Spurenelementen als Zusätze zu AiO-Mischungen wird empfohlen, allerdings setzt sie nachgewiesene Kompatibilität und Stabilität voraus.

\section{Empfehlung 39:}

Im Rahmen einer individuellen Rezeptur hergestellte AiO-Nährmischungen zur HPE sollen unter strikt aseptischen Bedingungen in der Regel zentral in der Apotheke gemäß der Leitlinie zur Qualitätssicherung der Bundesapothekerkammer zubereitet werden, wobei die anerkannten pharmazeutischen Regeln und regulatorischen Anforderungen zu beachten sind.

[A; starker Konsens]

Kommentar: Das Compounding, d.h. die Zubereitung von AiONährmischungen auf Basis individueller Rezepturen, wird seit der Etablierung von standardisierten parenteralen Nährmischungen nur vereinzelt durchgeführt, z. B. bei Kindern oder in der Langzeit-HPE, z.B. bei Kurzdarmsyndrompatienten $[177,227]$.

Die Zubereitung muss in jedem Schritt definierte pharmazeutische Herstellungsvorschriften (standard operation procedures, SOP) erfüllen [222-225]. Die Beurteilung der kritischen mikrobiologischen (aseptische Zubereitung) und physikochemischen Stabilität (Emulsionsdispersität, Löslichkeiten, Zersetzung, Sorptionsphänomene usw.) erfordern ein spezifisches pharmazeutisches Fachwissen; sie beeinflussen die Qualität der PE [228-230]. Zur Bestimmung kompatibler und damit zulässiger Elektrolytdosierungen sind Löslichkeitskurven oder spezifische Daten zur Emulsionsdestabilisierung nützliche Hilfsmittel [228, 231].

An die Herstellung von AiO-Nährmischungen werden aufgrund der Unmöglichkeit einer Endsterilisation, der hohen Anzahl enthaltener Komponenten, der Problematik physikochemischer Inkompatibilitäten und Instabilitäten hohe Anforderungen gestellt. Die mikrobiologische Stabilität aseptisch hergestellter AiO-Nährmischungen muss über die Anwendungsdauer gewährleistet sein [227, 232 - 235]. Es müssen die Kompatibilität der Einzelkomponenten, der pH-Wert, die Homogenität der Emulsion geprüft und bei der Herstellung eine Gewichtsprüfung (Soll-Ist-Kontrolle) vorgenommen werden. Ebenso muss die Maximalmenge der 
ausgewählten und als kompatibel getesteten (oder via Literaturrecherche als kompatibel bekannten) Zusätze definiert werden. Das Arbeiten unter streng aseptischen Bedingungen ist unumgänglich. Dies erfordert neben der notwendigen sächlichen (Laminar-Airflow-Werkbänke, Reinräume, „Compounder“) und personellen Infrastruktur eine Validierung des Herstellungsprozesses mit geeigneten Maßnahmen zur Qualitätskontrolle und Qualitätssicherung (z. B. systematische Überwachung und Dokumentation der Produktionshygiene, Hygieneplan sowie ein etabliertes Qualitätsmanagementsystem). Die anerkannten pharmazeutischen Regeln und regulatorischen Vorgaben sind zu beachten [222 - 225, 236]. Die A-Empfehlung basiert auf dem regulatorischen bzw. legalen Charakter der Empfehlung.

\section{Empfehlung 40:}

AiO-Nährmischungen oder Ernährungsprodukte zur HPE sollten aufgrund der komplexen Interaktionsmöglichkeiten in der Regel nicht als Träger für Medikamente verwendet werden. Wenn in Ausnahmefällen Medikamentenzusätze notwendig sind, sollte eine Dokumentation von Stabilität und Wirksamkeit empfohlen werden. [KKP; starker Konsens]

Kommentar: Aufgrund der zahlreichen Inhaltsstoffe von AiONährmischungen (Lipidemulsion, Aminosäuren, Glukose, Elektrolyte, Spurenelemente, Vitamine) sind Instabilitäten und physikochemische Inkompatibilitäten häufig [237, 238]. Beispielsweise können bei lipophilen Arzneistoffen in Emulsionssystemen veränderte Strukturen entstehen, deren Pharmakokinetik sich von der Ausgangssubstanz klinisch relevant unterscheidet oder Veränderungen der Verfügbarkeit auch der Substrate bewirken [239]. Vor jeder Zumischung eines Medikaments ist daher eine detaillierte Recherche oder analytische Abklärung erforderlich. Solche Zumischungen sollten die Ausnahme bilden.

\section{Empfehlung 41:}

Laut Verordnung sollen AiO-Nährmischungen als Rezepturanfertigung korrekt beschriftet sein, um die Arzneimitteltherapiesicherheit zu gewährleisten. Die Vorgaben der Apothekenbetriebsordnung (§14) zur Kennzeichnung von Rezepturarzneimitteln soll eingehalten werden.

[A; starker Konsens]

Kommentar: Die vollständige Beschriftung von Rezepturarzneimitteln gemäß den Vorgaben der Kennzeichnungspflicht nach $\S 14$ Apothekenbetriebsordnung dient der Vermeidung von Fehlapplikationen, zur sachgerechten Kontrolle und Dokumentation der Applikation und somit der Arzneimitteltherapiesicherheit [240]. Die Beschriftung soll daher zum einen die Patientendaten (Name, Geburtsdatum, ggf. Körpergewicht) enthalten, zum anderen müssen die Produktdaten aufgeführt werden. Dies umfasst 1) Name und Anschrift der abgebenden Apotheke und, soweit unterschiedlich, des Herstellers, 2) Inhalt nach Gewicht, Rauminhalt oder Stückzahl, 3) Art der Anwendung und Gebrauchsanweisung, 4) Angabe der Wirkstoffe nach Art und Menge und sonstige Bestandteile nach der Art, 5) Herstellungsdatum, 6) Verwendbarkeitsfrist mit dem Hinweis „verwendbar bis“ unter Angabe von Tag, Monat und Jahr, soweit erforderlich auch Angabe der Haltbarkeit nach Herstellung der gebrauchsfertigen Zubereitung, 7) soweit erforderlich, Hinweise auf besondere Vorsichtsmaßnahmen für die Aufbewahrung oder für die Beseitigung von nicht verwendeten Arzneimitteln oder sonstige besondere Vorsichtsmaßnahmen, um Gefahren für die Umwelt zu vermeiden. Die Angaben müssen in gut lesbarer Schrift, auf dauerhafte Weise angebracht und in deutscher Sprache verfasst sein. Soweit für das Rezepturarzneimittel Fertigarzneimittel als Ausgangsstoffe eingesetzt werden, genügt anstelle der Wirkstoffangaben die Angabe der Bezeichnungen der eingesetzten Fertigarzneimittel. Die Angaben zur Aufbewahrung oder Beseitigung können auch in einem Begleitdokument gemacht werden. Standardisierte und gedruckte Etiketten zur Kennzeichnung der AiO-Nährmischungen als Rezepturarzneimittel stellen eine sinnvolle und praktikable Lösung dar. Angaben zu Energie- und Eiweißgehalt der AiO-Nährmischung sind wünschenswert. Die A-Empfehlung basiert auf dem regulatorischen bzw. legalen Charakter der Empfehlung.

\section{Organisation und Überwachung der künstlichen Er- nährung im ambulanten Bereich und in Pflegeheimen}

$\nabla$

\section{Empfehlung 42:}

Die HEE und HPE sollte standardisiert und koordiniert möglichst durch ein interdisziplinäres Ernährungsteam erfolgen, weil dadurch die Qualität der Maßnahmen erhöht, die Komplikationsraten reduziert und somit ein entscheidender Beitrag zur Verbesserung der Lebensqualität der Patienten und zur Kosteneffizienz der Maßnahmen erreicht werden kann.

[B; starker Konsens]

Kommentar: Ernährungsteams (NST, nutrition support teams) sind der Zusammenschluss interdisziplinär tätiger und interprofessioneller Fachkräfte für künstliche Ernährung mit definierter Aufgabenverteilung, die sich in der Praxis etabliert hat ( $\bullet$ Tab. 1). Sie können den komplexen Anforderungen in der Versorgung von Patienten mit HEE und HPE-Patienten aufgrund ihrer breiten Expertise und ihrer Schnittstellenfunktion zwischen stationärer und ambulanter Patientenbetreuung am ehesten gerecht werden [5-9, $241-243]$. Deshalb sollte insbesondere die langfristige ernährungsmedizinische Überwachung durch ein solches Team erfolgen, welches die Effizienz einer Ernährungstherapie bezogen auf spezifische Endpunkte überwacht. Die meisten Ernährungsteams der deutschen Kliniken sind sowohl im ambulanten bzw. Überleitungs- als auch im stationären Bereich tätig [244]. Sie können Handlungsabläufe bei der künstlichen Ernährung effizient und kostengünstig gestalten, Komplikationsraten verringern und die Lebensqualität der betroffenen chronisch kranken Patienten verbessern [245, 246]. Die Organisation der HEE bzw. HPE sollte bei stationären Patienten vor Entlassung aus dem Krankenhaus erfolgen und erfordert meist etwa 1 - 2 Tage Vorbereitung. Bei initial ambulanten Patienten wird die HEE oder HPE entweder durch Haus- bzw. Fachärzte oder durch involviertes Pflegepersonal der häuslichen Pflege oder in Pflegeeinrichtungen initiiert [5]. Dazu ist eine klare Zuordnung von Zuständigkeiten notwendig, wodurch die Zusammenarbeit des Ernährungsteams mit ambulanten Pflegediensten, Apotheken und Homecare-Versorgern geregelt wird ( Tab.1). Der wirtschaftliche Vorteil durch Einrichtung eines Ernährungsteams bzw. durch die Tätigkeit einer Ernährungspflegekraft in Krankenhäusern konnte in mehreren Studien belegt werden [247-251]. Allerdings gibt es zur ersten Aussage auch relativierende Ergebnisse, je nachdem, welche Outcome-Variablen betrachtet werden [252]. Dennoch werden interdisziplinäre Ernährungsteams weltweit 
Tab.1 Zuständigkeiten bei der Organisation und Durchführung der HEE/HPE.

\begin{tabular}{|c|c|}
\hline Einrichtung & Zuständigkeit/Aufgaben \\
\hline $\begin{array}{l}\text { Ernährungsteam für künstli- } \\
\text { che Ernährung, bestehend } \\
\text { aus Team-Arzt, Diätassis- } \\
\text { tent, Ernährungswissen- } \\
\text { schaftler, Apotheker und } \\
\text { Pflegekraft }\end{array}$ & $\begin{array}{l}\text { - Festlegung der ernährungsmedizinischen Maßnahme in Kooperation mit dem behandelnden Arzt } \\
\text { - Ernährungsanamnese, Kostaufbau nach Beendigung der künstlichen Ernährung, supplementäre Trinknahrung } \\
\text { - gegebenenfalls individuelle Ernährungsberatung und Schulung von Patienten bzw. dessen Angehörigen bzgl. der } \\
\text { Selbstversorgung mit HEE oder HPE, sofern dies gewünscht wird } \\
\text { - Wahl des geeigneten Zugangs, Berechnung und Rezeptieren der HEE bzw. HPE sowie notwendiger Hilfsmittel (Arzt) } \\
\text { - mündliche und schriftliche Aufklärung über Art, Umstände und typische Komplikationen der häuslichen Versorgung } \\
\text { - Auswahl, Beauftragung und Überprüfung eines Homecare-Versorgers bzw. anderer beteiligter Institutionen/Personen } \\
\text { (z. B. Brückenpflege, Hausarzt, Pflegedienst, Apotheke) } \\
\text { - Überwachung der HEE und HPE, ggf. in enger Kooperation mit dem jeweiligen Hausarzt, Management von Komplika- } \\
\text { tionen }\end{array}$ \\
\hline Ambulanter Pflegedienst & $\begin{array}{l}\text { - gegebenenfalls Zuspritzen von Mikronährstoffen unmittelbar vor Anhängen der parenteralen Ernährungsinfusionen } \\
\text { - An-und Abhängen der HEE bzw. HPE (nach Schulung kann dies ggf. vom Patienten/Angehörigen übernommen werden) } \\
\text { - Wechseln der Portnadel bzw. Verabreichung von Medikamenten über Sonde oder Katheter bei Vorliegen einer } \\
\text { Delegationsvereinbarung durch den betreuenden Arzt } \\
\text { - gegebenenfalls Wundversorgung nach Sonden- bzw. Katheteranlage }\end{array}$ \\
\hline Apotheke & $\begin{array}{l}\text { - Evaluation/Bestellung und Auslieferung der Produkte in verwendungsgeeigneter Form mit Hinweisen zur Handhabung } \\
\text { - Überprüfung der Stabilitätsdaten und Qualitätssicherung (Dokumentation) } \\
\text { - gegebenenfalls Zusammenmischen (Compounden) der Infusionslösung nach Vorgaben eines Arztes } \\
\text { - Beratung und Empfehlung zur Durchführung der Begleitmedikation }\end{array}$ \\
\hline Homecare-Versorger & $\begin{array}{l}\text { - Schulung des Pflegedienstes bzw. des Patienten oder seiner Angehörigen bezüglich des Umgangs mit dem jeweiligen } \\
\text { Ernährungssystem und der Erkennung von Komplikationen } \\
\text { - Organisation der Belieferung } \\
\text { - regelmäßige Patientenbesuche mit schriftlicher Dokumentation der Verlaufskontrollen, ggf. in Kooperation mit dem } \\
\text { Pflegedienst } \\
\text { - regelmäßiger Bericht an das Ernährungsteam }\end{array}$ \\
\hline
\end{tabular}

nicht zuletzt wegen ihres wirtschaftlichen Vorteils in Positionspapieren und Leitlinien empfohlen [5 - 9, 253-256]. Es ist davon auszugehen, dass diese positiven Effekte auch und gerade im ambulanten Bereich zum Tragen kommen. Aufgrund des Analogiecharakters dieser Schlussfolgerung wird der Evidenzgrad von A nach B abgestuft.

\section{Empfehlung 43:}

Die Beauftragung zur Mitbehandlung durch ein interdisziplinäres Ernährungsteam soll durch den behandelnden Arzt (Krankenhausarzt oder ambulant tätiger Arzt) erfolgen und sollte in Zusammenarbeit mit dem zuständigen Hausarzt fortgesetzt werden.

[KKP; starker Konsens]

Kommentar: Die ärztliche Beauftragung kann die Durchführung der HEE oder HPE umfassen, wenn die Indikation zur künstlichen Ernährung bereits gestellt wurde, oder die Prüfung der Indikation einschließen, wenn die Indikation noch nicht gestellt wurde. Das Stellen der Indikation sowie die Rezeptierung der künstlichen Ernährung müssen aufgrund gesetzlicher Bestimmungen durch einen Arzt erfolgen. Das Ernährungsteam sollte in die Entscheidung zur künstlichen Ernährung besonders dann einbezogen werden, wenn es sich um Grenzfälle und Individualabwägungen handelt. Der Hausarzt sollte über die Art der jeweiligen Therapie und die beteiligten Institutionen und Organisationsstrukturen (z. B. Ernährungsteam, Homecare-Provider oder Pflegedienst) von Beginn der häuslichen Versorgung an informiert werden. In Absprache mit den betreuenden Institutionen kann ein großer Teil des Monitorings vom Hausarzt durchgeführt werden (z. B. Laborkontrollen). Die Ergebnisse sollten zeitnah an das Ernährungsteam bzw. andere beteiligte Institutionen übermittelt werden.

\section{Empfehlung 44:}

Bei Patienten unter HEE oder HPE sollten regelmäßig klinische und laborchemische Verlaufskontrollen durchgeführt werden, einerseits zur Evaluation der Effektivität der ernährungsmedizinischen Maßnahmen und andererseits zur Reduktion von ernährungsassoziierten Komplikationen.

[B; starker Konsens]

Kommentar: Die ernährungsmedizinische Überwachung von mit HEE oder HPE behandelten Patienten ist notwendig, um Veränderungen des klinischen Zustands zu erfassen und ggf. die getroffenen Maßnahmen anzupassen bzw. einzustellen (siehe auch die aktuelle DGEM-Leitlinie „Besonderheiten der Überwachung bei künstlicher Ernährung“ [3]). Trotz generell eingeschränkter Studienlage hinsichtlich der Evidenzgrade erhielt diese Empfehlung einen Grad B, weil aufgrund ethischer Gesichtspunkte kontrollierte prospektive Studien mit höherer Evidenz kaum durchführbar sind. Die Verlaufskontrollen sollten möglichst durch ein interdisziplinäres Ernährungsteam erfolgen bzw. koordiniert werden [242].

Die Effektivität einer Ernährungstherapie sollte auf spezifische Endpunkte bezogen werden, deren Definition sich nach der Grunderkrankung des Patienten, seinem klinischen Zustand, den Möglichkeiten der betreuenden Institution und nicht zuletzt nach den individuellen Wünschen des Patienten richten [257]. Solche Endpunkte können die Aufrechterhaltung oder Wiederherstellung des Körperproteinbestands, die Senkung der Morbidität und Mortalität, die Verbesserung von etablierten Surrogatparametern und insbesondere von Lebensqualität beinhalten [258].

Einige Studien haben die Lebensqualität bei HPE-Patienten evaluiert und gezeigt, dass die HPE einen positiven Einfluss auf das psychosoziale Wohlbefinden sowohl der Patienten als auch der Familienangehörigen haben kann [259]. In der Studie von Carlson et al. [260] berichteten allerdings 40,5\% der HPE-Patienten über 
signifikante psychologische Belastung, wobei Verdruss, Angst und Depression häufiger vorkommen. Bei den Patienten mit reaktiver Depression wurden höhere Raten von lebensbedrohlichen Komplikationen beobachtet [261, 262], außerdem wurden Einschränkungen beim Reisen und im allgemeinen Sozialleben bemängelt [262, 263]. Diverse Studien zeigten, dass eine Verbesserung der Lebensqualität bei mangelernährten Patienten mit der Optimierung des Ernährungsstatus einhergeht [264-266]. Verschiedene Fachgesellschaften empfehlen deshalb die regelmäßige Erfassung der Lebensqualität bei HPE-Patienten, beispielsweise mit dem „SF-36“, dem „EuroQOL“ oder dem „Karnofsky Score“. Die Forschungsgruppe von Baxter et al. [267] hat einen spezifischen Fragebogen (HPN-QOL) für die Erfassung der Lebensqualität bei HPE-Patienten entwickelt und validiert. Bei Krebspatienten ist auch der „International Classification of Functioning, Disability and Health“ (ICF) als Klassifikationssystem geeignet [268]. Die Reduktion von Komplikationen durch adäquate Betreuung und Verlaufskontrollen wurde für HEE $[269,270]$ und HPE $[271,272]$ gezeigt.

\section{Empfehlung 45:}

Die Intervalle zwischen den Verlaufskontrollen können bei stabilen HEE- und HPE-Patienten im ersten Quartal wöchentlich erfolgen, danach mindestens alle 3 Monate. Bei klinisch instabilen Patienten sollten kürzere Intervalle gewählt werden.

[KKP; starker Konsens]

Kommentar: Es liegen keine kontrollierten Studien vor, in denen der Outcome von HEE oder HPE hinsichtlich der Intervalle von Verlaufskontrollen untersucht wurden. Insofern handelt es sich hier allein um Erfahrungswerte, die sich in der klinischen Praxis bewährt haben und von Experten bestätigt wurden.

\section{Empfehlung 46:}

Ernährungsanamnese, Körpergewicht, Sonden- bzw. Kathetereintrittsstelle, Hydradationszustand und wichtige biochemische Parameter wie Glukose, Elektrolyte, CRP, Nieren- und Leberfunktionsparameter sollten mindestens quartalsweise als Verlaufskontrolle erhoben werden.

[B; starker Konsens]

Kommentar: Zur Frage, welche Parameter bei wem im Verlauf der HEE oder HPE erhoben werden sollen, gibt es wenig Literatur. - Tab.2 fasst Angaben aus Berichten von Expertenausschüssen, publizierten Erfahrungsberichten und Expertenmeinungen zusammen [6-10, 197, 218, 243, 273 -276]. Je nach Grunderkrankung, Komorbiditäten und Krankheitsverlauf müssen die Kontrolluntersuchungen durch das behandelnde, klinische Ernährungsteam und involvierte Hausärzte sowie Spezialisten angepasst und entsprechend verordnet werden. In der Praxis hat sich eine mindestens vierteljährliche Kontrolle der in der Empfehlung genannten Parameter bewährt. Die Messung von Vitaminen, Mineralstoffen und weiteren Mikronährstoffen sollte möglichst alle 6-12 Monate durchgeführt werden, falls daraus Aussagen zu allfälligen Unter- oder Überdosierungen ableitbar sind. Die Überwachung und Kontrolle der Knochendichte mittels Dual-EnergyAbsorptiometrie (DXA) sollte je nach Verfügbarkeit alle 1-2 Jahre wiederholt werden.

Drei prospektive Studien untersuchten die Effizienz einer ernährungsmedizinischen Überwachung bezüglich Prognose und Kos- ten [277-279]. Unter einer solchen Verlaufskontrolle zeigte sich eine signifikante Abnahme an Komplikationen und Kosten im Vergleich zu Populationen ohne Verlaufskontrolle. Sie ermöglicht die Anpassung der Zusammensetzung der Nährmischungen an Veränderungen des klinischen Zustands, des Aktivitätsniveaus und der Laborwerte. Bei unbefriedigendem Verlauf kann die indirekte Kalorimetrie herangezogen werden, um präzise Veränderungen im Energiebedarf und -umsatz zu erfassen. Ebenfalls sollte in regelmäßigen Abständen kontrolliert werden, ob die Indikation zur HEE oder HPE weiterbesteht.

\section{Empfehlung 47:}

Bereits vor der Entlassung sollte nach Möglichkeit eine Schulung des Patienten und/oder seiner Angehörigen (u. a. zur Zubereitung der Nährlösung, Bedienung der Technik, Hygiene, Maßnahmen bei Problemen) durch qualifiziertes Personal erfolgen und im Verlauf hinsichtlich seines Erfolgs überprüft bzw. wiederholt werden. [B; starker Konsens]

Kommentar: Unzureichende Sicherheit im Umgang mit der Nahrung und der Applikationstechnik kann zu relevanten Komplikationen und zur Wiederaufnahme in die Klinik führen. Auf der anderen Seite erhöhen mehrfache Schulungen unter Einsatz visueller Hilfsmittel, die dem Patienten zur Verfügung gestellt werden, die Effizienz der Ernährungstherapie [246, 280, 281] und führen zu höherer Lebensqualität durch Unabhängigkeit und Sicherheit [270]. Nachschulungen sind meist erforderlich, da sich im Laufe der Zeit zu Hause Fehler einschleichen und Schulungsinhalte vergessen werden [282]. Die Schulungen sollten durch Fachkräfte erfolgen, die eine ernährungsmedizinische Zusatzqualifikation und Erfahrung vorweisen können [283]. Dies ist im günstigen Fall ein ambulant tätiges interdisziplinäres Ernährungsteam (vgl. Empfehlung 42).

\section{Empfehlung 48:}

Der Patient kann sich die Nährlösungen nach adäquater Schulung selbst an- und abhängen, was ihm eine zeitliche Unabhängigkeit von Fachpersonal ermöglicht und eine Verbesserung seiner Lebensqualität bewirken kann.

[KKP; starker Konsens]

Kommentar: Meist erfolgt das An- und Abhängen der enteralen bzW. parenteralen Ernährung durch hierfür besonders geschultes Pflegepersonal des Homecare-Providers oder des Pflegedienstes. Es ist aber auch möglich, den Patienten selbst bzw. dessen Angehörige bezüglich der Zufuhr der enteralen bzw. parenteralen Ernährung zu unterweisen. So sind die Patienten nicht mehr vom Zeitplan des jeweiligen Pflegedienstes abhängig und freier in der Gestaltung ihres Tagesablaufs.

\section{Empfehlung 49:}

Der Kostaufbau nach HEE oder HPE sollte stufenweise und individuell unter Anleitung einer Ernährungsfachkraft durchgeführt werden; er kann nach standardisierten Konzepten erfolgen, sollte sich aber sowohl an der gastrointestinalen Toleranz als auch der Fähigkeit zur oralen Nahrungsaufnahme orientieren und mit einer entsprechenden Reduktion der künstlichen Ernährung einhergehen. [KKP; starker Konsens] 
Tab.2 Verlaufskontrollen bei künstlicher Ernährung ( $E=H E E ; P=H P E)$.

\begin{tabular}{|c|c|c|c|c|c|c|c|c|c|c|}
\hline \multicolumn{7}{|l|}{ Kontrollen im ersten Jahr } & \multicolumn{4}{|c|}{ langfristige Kontrollen } \\
\hline Parameter & Wo. 0 & Wo. 1 & Wo. 2 & Wo. 4 & Wo. 6 & Wo. 8 & Mo. 3 & Mo. 6 & Mo. 9 & jährlich \\
\hline \multicolumn{11}{|l|}{ Anamnese } \\
\hline Ernährungsanamnese & $E / P$ & $P$ & $P$ & $E / P$ & $P$ & $P$ & $E / P$ & $E / P$ & $E / P$ & $E / P$ \\
\hline $\begin{array}{l}\text { Erfassung von Energie-, Substrat- } \\
\text { und Flüssigkeitsbedarf }\end{array}$ & $E / P$ & & & & $P$ & & $E / P$ & $E / P$ & $E / P$ & $E / P$ \\
\hline Erfassung der Lebensqualität & $P$ & & & & & & & & & $P$ \\
\hline \multicolumn{11}{|l|}{ klinische Kontrolle } \\
\hline Körpergewicht, -länge & $E / P$ & $P$ & $P$ & $E / P$ & $P$ & $P$ & $E / P$ & $E / P$ & $E / P$ & $E / P$ \\
\hline $\begin{array}{l}\text { Vitalfunktionen (Temperatur, RR, } \\
\text { Puls) }\end{array}$ & $P$ & $P$ & $P$ & $P$ & $P$ & $P$ & $P$ & $P$ & $\mathrm{P}$ & $E / P$ \\
\hline $\begin{array}{l}\text { PEG-bzw. ZVK- oder Port-Eintritts- } \\
\text { stelle }\end{array}$ & $E / P$ & $E / P$ & $\mathrm{E} / \mathrm{P}$ & $E / P$ & $E / P$ & $\mathrm{E} / \mathrm{P}$ & $E / P$ & $E / P$ & $E / P$ & $E / P$ \\
\hline Hydratationszustand & $E / P$ & & & $E / P$ & & $P$ & $\mathrm{E} / \mathrm{P}$ & $E / P$ & $\mathrm{E} / \mathrm{P}$ & $E / P$ \\
\hline kardiopulmonaler Status & $P$ & & & $\mathrm{P}$ & & $P$ & $\mathrm{P}$ & $\mathrm{P}$ & $P$ & $E / P$ \\
\hline abdominaler Status & $P$ & & & $\mathrm{P}$ & & $P$ & $\mathrm{P}$ & $\mathrm{P}$ & $P$ & $E / P$ \\
\hline $\begin{array}{l}\text { Haut, Haare, Nägel, Wunden, } \\
\text { Schleimhäute }\end{array}$ & $\mathrm{P}$ & & & $\mathrm{P}$ & & $P$ & $\mathrm{P}$ & $P$ & $P$ & $E / P$ \\
\hline Nutrition Risk Score (NRS-2002) & $E / P$ & & & $E / P$ & & & $E / P$ & $E / P$ & $\mathrm{E} / \mathrm{P}$ & $\mathrm{E} / \mathrm{P}$ \\
\hline $\begin{array}{l}\text { Anthropometrie (OAU, HF, Kraft, } \\
\text { BIA etc.) }\end{array}$ & $E / P$ & & & $E / P$ & & & $E / P$ & $E / P$ & $E / P$ & $E / P$ \\
\hline \multicolumn{11}{|l|}{ Laborparameter } \\
\hline Glukose & $E / P$ & $P$ & $P$ & $E / P$ & $P$ & $P$ & $E / P$ & $E / P$ & $E / P$ & $E / P$ \\
\hline Natrium, Kalium & $\mathrm{E} / \mathrm{P}$ & $P$ & $\mathrm{P}$ & $\mathrm{P}$ & $\mathrm{P}$ & $P$ & $E / P$ & $\mathrm{P}$ & $\mathrm{E} / \mathrm{P}$ & $E / P$ \\
\hline Kalzium, Magnesium, Phosphat & $E / P$ & $\mathrm{P}$ & $P$ & $\mathrm{P}$ & $P$ & $\mathrm{P}$ & $E / P$ & $\mathrm{P}$ & $E / P$ & $E / P$ \\
\hline CRP & $E / P$ & $P$ & $P$ & $\mathrm{P}$ & $P$ & $P$ & $\mathrm{P}$ & $\mathrm{P}$ & $P$ & $E / P$ \\
\hline Kreatinin, Harnstoff & $E / P$ & $P$ & $P$ & $\mathrm{P}$ & $P$ & $P$ & $\mathrm{P}$ & $P$ & $\mathrm{P}$ & $E / P$ \\
\hline venöse Blutgasanalyse & $P$ & & $P$ & & $P$ & & $P$ & $P$ & $\mathrm{P}$ & $P$ \\
\hline AST, ALT, GGT, AP & $P$ & & $P$ & & $P$ & & $P$ & $P$ & $P$ & $E / P$ \\
\hline Triglyzeride & $P$ & & $P$ & & $P$ & & $P$ & $P$ & $\mathrm{P}$ & $\mathrm{P}$ \\
\hline Protein, Albumin & $E / P$ & & & & $\mathrm{P}$ & & $E / P$ & $\mathrm{P}$ & $E / P$ & $E / P$ \\
\hline Blutbild & $E / P$ & & & & $P$ & & $P$ & $P$ & $P$ & $E / P$ \\
\hline Bilirubin, Amylase & $P$ & & & & & & & $\mathrm{P}$ & & $\mathrm{P}$ \\
\hline Harnsäure & $P$ & & & & & & & $\mathrm{P}$ & & $\mathrm{P}$ \\
\hline Quick od. INR (Vit. K) & $E / P$ & & & & & & & $P$ & & $E / P$ \\
\hline Folat, Vit. $B_{12}$, Vit. D & $\mathrm{E} / \mathrm{P}$ & & & & & & & $\mathrm{P}$ & & $E / P$ \\
\hline Ferritin, Zn, Cu, Se, Mg & & & & & & & & $P$ & & $E / P$ \\
\hline Vit. A, Vit. E, Vit. B & & & & & & & & & & $\mathrm{P}$ \\
\hline Parathormon & & & & & & & & & & $P$ \\
\hline \multicolumn{11}{|l|}{ Urinparameter/24h } \\
\hline $\begin{array}{l}\text { Krea, Na, Ca, Hs, Protein, Oxalat, } \\
\text { Citrat }\end{array}$ & & & & & & & & & & $P$ \\
\hline \multicolumn{11}{|l|}{ spezielle Untersuchungen } \\
\hline $\begin{array}{l}\text { Knochendensitometrie (DXA) } \\
\text { (1-2 jährlich) }\end{array}$ & & & & & & & & & & $P$ \\
\hline Abdominalultraschall & - & - & - & - & - & - & - & - & & $\begin{array}{l}\text { nach } \\
\text { Bedarf }\end{array}$ \\
\hline $\begin{array}{l}\text { Blutkulturen (ZVK, peripher), } \\
\text { Abstrich }\end{array}$ & - & - & - & - & - & - & - & - & & $\begin{array}{l}\text { nach } \\
\text { Bedarf }\end{array}$ \\
\hline
\end{tabular}

Kommentar: Es gibt keine Studien, welche die Durchführung des Kostaufbaus als primäre Fragestellung untersucht haben. Demzufolge bestehen auch keine Standardprotokolle für den Kostaufbau. Es lassen sich lediglich Hinweise in der Literatur finden, die den Einsatz krankheitsadaptierter diätetischer Maßnahmen wie z.B. mehrere kleine Mahlzeiten, mittelkettige Fettsäuren bei Digestions- und Resorptionsstörungen als vorteilhaft erwähnt haben. Darüber hinaus sind Vorlieben, Unverträglichkeiten, soziale und finanzielle Gesichtspunkte einzubeziehen. Gerade die Adaptationsphase verlangt eine intensive ernährungsmedizinische Betreuung des Patienten mit engmaschigen Konsultationen durch einen ernährungsmedizinisch erfahrenen Arzt oder eine
Ernährungsfachkraft (z.B. Diätassistenten, Diätologen, Ernährungswissenschaftler mit klinischer Erfahrung).

In jedem Fall muss beim Kostaufbau die Grunderkrankung berücksichtigt werden. Dies gilt z. B. für auftretende Schmerzen bei Pankreatitis und Diarrhöen beim Kurzdarmsyndrom. Entscheidend ist die ausreichende Zufuhr von Energie und Nährstoffen. Darüber hinaus ist mit Stoffwechselimbalancen zu rechnen, die sowohl die Elektrolyte als auch die Blutglukose betreffen können. Bei der Umstellung von der enteralen auf die orale Nahrungszufuhr müssen eine ggf. vorliegende Beeinträchtigung der Schluckfähigkeit bzw. motorische Defizite berücksichtigt werden. Hierzu sollten bei Bedarf Logopäden und Ergotherapeuten hinzugezo- 
gen werden. Die Umstellung sollte stufenweise erfolgen, um die ausreichende Versorgung mit Energie und Nährstoffen sicherzustellen. Die permanente enterale Sonde sollte nicht zu früh entfernt werden. Sie kann ggf. ohne Beschickung mit Nährlösung auch für mehrere Wochen belassen werden, bis der orale Kostaufbau erfolgreich abgeschlossen ist.

\section{Empfehlung 50:}

Sondenernährung und parenterale Ernährung können bei Pflegeheimbewohnern unter denselben Voraussetzungen durchgeführt werden wie bei älteren Menschen im Privathaushalt bzw. bei jüngeren Personen.

[KKP; starker Konsens]

Kommentar: Einzelheiten dazu siehe S3-Leitlinie der DGEM „Klinische Ernährung in der Geriatrie“ [1].

\section{Empfehlung 51:}

In Pflegeheimen sollten geeignete Struktur- und Prozessanforderungen erfüllt sein, um eine adäquate ernährungsmedizinische Versorgung zu ermöglichen.

[B; starker Konsens]

Kommentar: Ältere Menschen in Pflegeheimen haben aufgrund häufiger funktioneller und gesundheitlicher Beeinträchtigungen im Vergleich zu selbstständig lebenden Senioren und jüngeren Personen ein besonders hohes Risiko für Ernährungsprobleme und Mangelernährung. Einer internationalen Datenbankanalyse zufolge waren $14 \%$ von mehr als 4500 Pflegeheimbewohnern von Mangelernährung betroffen, 53\% hatten ein Risiko für Mangelernährung [284]. Daher benötigen Pflegeheimbewohner erhöhte Aufmerksamkeit zur Sicherung einer ausreichenden Energie- und Nährstoffzufuhr.

Bei bestehendem Risiko für Mangelernährung bzw. bestehender Mangelernährung müssen frühzeitig individuell an die jeweilige Problemlage und Ressourcen angepasste Maßnahmen erfolgen, um eine ausreichende Energie- und Nährstoffzufuhr zu sichern und den Ernährungszustand zu verbessern bzw. zu erhalten. Hierzu steht ein breites Spektrum möglicher Interventionen zur Verfügung, die sich gegenseitig nicht ausschließen, sondern ergänzen und auch kombiniert zum Einsatz kommen sollen (๑ Tab.3).

In zahlreichen Studien sind positive Effekte verschiedener Maßnahmen zur Verbesserung der Ernährungssituation im Pflegeheim, z. B. Umgebungsgestaltung, pflegerische Förderung, Modifikation der Mahlzeiten, im Hinblick auf die Energie- und Nährstoffzufuhr und den Ernährungszustand belegt. Am besten untersucht sind die Effekte von Trinknahrung, deren Einnahme bei älteren Menschen in Langzeitpflege im Rahmen einer Metaanalyse mit einer signifikant besseren Entwicklung des Körpergewichts (WMD 2,51; 95\%-KI 1,73-3,28) und einer grenzwertig reduzierten Mortalität (OR 0,65; 95\%-KI 0,41-1,02) verbunden war [285].

Zu den geeigneten Struktur- und Prozessanforderungen in Pflegeheimen gehören ein attraktives Esszimmer für gemeinsame Mahlzeiten, ausreichend Zeit für die Mahlzeiten, gut organisierte Pflegeabläufe und eine angemessene Personalausstattung, um eine hochwertige Ernährungsversorgung zu ermöglichen. Im Pflegeheim sollen generell alle Optionen der Ernährungstherapie zur Verfügung stehen und in Abhängigkeit vom Ernährungs- und
Tab.3 Spektrum der Ernährungstherapie bei älteren Menschen im Pflegeheim.

\begin{tabular}{|ll}
\hline Maßnahmen & Beispiele \\
\hline $\begin{array}{l}\text { Beseitigung zugrunde } \\
\text { liegender Ursachen }\end{array}$ & $\begin{array}{l}\text { Verzicht auf restriktive Diäten, Zahnsanie- } \\
\text { rung, Schlucktraining, adäquate Behandlung } \\
\text { der Grunderkrankung }\end{array}$ \\
\hline $\begin{array}{l}\text { angenehme Essumge- } \\
\text { bung }\end{array}$ & $\begin{array}{l}\text { Essen am Tisch in einem gemeinsamen } \\
\text { Esszimmer, angenehme Atmosphäre }\end{array}$ \\
\hline Pflegemaßnahmen & $\begin{array}{l}\text { verbale Aufforderung, Essen schneiden, } \\
\text { Essen reichen }\end{array}$ \\
\hline $\begin{array}{l}\text { Modifikation der Mahl- } \\
\text { zeiten und Lebensmittel }\end{array}$ & $\begin{array}{l}\text { Wunschkost, Zwischenmahlzeiten, Finger- } \\
\text { food, Modifikation von Textur und Konsistenz }\end{array}$ \\
\hline Anreicherung & $\begin{array}{l}\text { Zugabe von Sahne, Butter, Öl, Ei oder von } \\
\text { Nährstoffkonzentraten, z. B. Proteinpulver, } \\
\text { Maltodextrin zu Speisen und Gerichten }\end{array}$ \\
\hline Trinknahrung & $\begin{array}{l}\text { nach indiviuellen Geschmacksvorlieben } \\
\text { künstliche Ernährung }\end{array}$ \\
$\begin{array}{l}\text { Sondenernährung oder parenterale Ernäh- } \\
\text { rung und Flüssigkeitsgabe (ergänzend/aus- } \\
\text { schließlich) }\end{array}$
\end{tabular}

Gesundheitszustand nach individueller Nutzen-Risiko-Abwägung zum Einsatz kommen. Dazu gehört auch, dass ein attraktives und abwechslungsreiches Angebot an Lebensmitteln und Zwischenmahlzeiten verfügbar ist und an die individuellen Bedürfnisse angepasst werden kann (z.B. Konsistenz, Fingerfood, Uhrzeit der Mahlzeiten) [285]. Einzelheiten dazu siehe S3-Leitlinie der DGEM „Klinische Ernährung in der Geriatrie“ [1].

\section{Empfehlung 52:}

Im Pflegeheim sollten standardisierte Handlungsempfehlungen zur Sicherung einer hochwertigen Ernährungsversorgung vorliegen, und Verantwortlichkeiten für die Ernährungstherapie klar geregelt sein.

[KKP; starker Konsens]

Kommentar: Im Pflegeheim sollen in regelmäßigen Abständen Screenings auf Mangelernährung erfolgen, um Ernährungsprobleme frühzeitig zu erkennen. Diese Handlungsempfehlung umfasst eine regelmäßige Erfassung des Ernährungszustands (Körpergewicht, alternativ Oberarmumfang) und von Ernährungsproblemen (z.B. ungenügende Nahrungsmenge, Unterstützungsbedarf) einschließlich deren mögliche Ursachen im Rahmen eines routinemäßigen Screening- und Assessmentprozesses [286]. Alle Optionen der Ernährungstherapie sollten zur Verfügung stehen und in Abhängigkeit vom Ernährungs- und Gesundheitszustand nach individueller Nutzen-Risiko-Abwägung zum Einsatz kommen. Weitere Einzelheiten dazu siehe S3-Leitlinie der DGEM „Klinische Ernährung in der Geriatrie“ [1].

\section{Empfehlung 53:}

Bei geriatrischen Patienten soll unter ambulanten Bedingungen eine leichte bis mittelschwere Dehydratation mittels Infusion von Flüssigkeit in das Subkutangewebe behandelt werden.

[A; starker Konsens]

Kommentar: Neben der Pneumonie ist die Dehydratation eine häufige Todesursache bei Pflegeheimbewohnern [287]. Die subkutane Infusion von Flüssigkeit wurde erstmals im Jahr 1913 bei Kindern mit infektiöser Diarrhö beschrieben [288]. Aktuell wird die subkutane Flüssigkeitsgabe vor allem in den Bereichen Geria- 
trie und Palliativmedizin angewandt. Die subkutane Flüssigkeitsgabe ist bei älteren Patienten mit leichter bis mittelschwerer Dehydratation genauso effektiv wie eine intravenöse Flüssigkeitsgabe [289]. Durch subkutane Flüssigkeitsgabe konnte in einer Studie bei $82 \%$ der Patienten eine ausreichende orale Nahrungsund Flüssigkeitsaufnahme erreicht werden, bei $84 \%$ der Patienten verbesserte sich die kognitive Funktion. Dies führte zu einer deutlichen Verbesserung in den Aktivitäten des täglichen Lebens, was durch eine Verbesserung des Barthel-Indexes dokumentiert werden konnte [290]. Der klinische Nutzen von subkutaner Flüssigkeitsgabe wurde in mehreren Studien belegt [289, 291].

Die Anwendung von subkutanen Infusionen kann Krankenhauseinweisungen verhindern und - verglichen mit der intravenösen Flüssigkeitsgabe - Kosten einsparen [289]. Besonders bei unruhigen und verwirrten Patienten und bei Patienten, bei denen intravenöse Punktionen nicht möglich sind, ist die subkutane Flüssigkeitsgabe eine sichere Alternative zur intravenösen Flüssigkeitsgabe. Allerdings ist eine subkutane Flüssigkeitsgabe in akuten Notfallsituationen mit der Notwendigkeit, rasch hohe Flüssigkeitsvolumina zu ersetzen, nicht indiziert, z. B. bei schwerer Dehydratation, bei Infusionsmengen von mehr als $3000 \mathrm{~mL}$ täglich, bei sehr schweren Gerinnungsstörungen und bei schweren Elektrolytentgleisungen. In diesen Fällen sollte die intravenöse Gabe bevorzugt werden.

\section{Empfehlung 54:}

Bei der Infusion von Flüssigkeit in das Subkutangewebe sollten folgende Punkte beachtet werden: 1) Die Art der Lösung sollte für subkutane Applikation geeignet sein; 2) über einen Zeitraum von 24 Stunden sollten nicht mehr als $2000 \mathrm{~mL}$ pro Infusionsstelle und insgesamt nicht mehr als $3000 \mathrm{~mL}$ pro 24 Stunden appliziert werden; 3) die Infusionsgeschwindigkeit sollte $125 \mathrm{~mL} /$ Stunde nicht überschreiten und 4) Hyaluronidase sollte in der Regel nicht verwendet werden.

[B; starker Konsens]

Kommentar: Als Infusionslösungen können 0,9\%ige Kochsalzlösung, halbisotonische Kochsalzlösung, 5\%ige Glukoselösung oder eine Mischung aus 5\%iger Glukose und 0,9\%iger Kochsalzlösung verwendet werden [289, 292]. Bei der Verwendung von 5\%iger Glukose sollten nicht mehr als $2000 \mathrm{~mL}$ innerhalb von 24 Stunden mit einer Infusionsgeschwindigkeit von nicht mehr als 2 $\mathrm{mL} /$ Minute verabreicht werden [293]. Es gibt Hinweise, dass bei der Verwendung von elektrolytfreien Lösungen häufiger Komplikationen auftreten als bei der Verwendung isotonischer Elektrolytlösungen. Von den anderen nichtisotonischen Lösungen sollten nicht mehr als $3000 \mathrm{~mL}$ innerhalb von 24 Stunden und nicht mehr als $2000 \mathrm{~mL}$ pro Injektionsstelle über 24 Stunden gegeben werden [293]. In einer Studie wurde auch die subkutane Gabe von Aminosäurelösungen mit der intravenösen Gabe von Aminosäurelösungen untersucht [294]. Da hierzu keine weiteren Studien zu Effektivität und Sicherheit einer subkutanen Gabe von Aminosäurelösungen existieren und die entsprechenden Lösungen keine Arzneimittelzulassung für die subkutane Gabe besitzen, wird die subkutane Gabe von Aminosäurelösungen in dieser Leitlinie nicht generell empfohlen.

Hyaluronidase ist ein Enzym, das die Konzentration der im Subkutangewebe vorkommenden Hyaluronsäure vermindert. Dadurch soll die Resorption subkutaner Flüssigkeit verbessert werden. Bruera et al. [295] untersuchten in einer randomisierten Cross-over-Studie bei 26 Patienten mit fortgeschrittenem Tumorleiden und Dehydratation die Gabe von Hyaluronidase in einer Dosierung von 150 und 300 Einheiten. Dabei fanden sich keine statistisch signifikanten Unterschiede bei Effektivität und Nebenwirkungen (lokale und systemische allergische Reaktionen) zwischen den beiden Dosierungen. Dieses Ergebnis wird von anderen randomisierten und nicht randomisierten kontrollierten Studien bestätigt $[292,296]$. Auf der anderen Seite existiert keine randomisierte Studie, die einen Vorteil der Hyaluronidaseapplikation zeigt. Dagegen kann Hyaluronidase Schmerzen und allergische Reaktionen verursachen [292]. Deshalb ist die Anwendung von Hyaluronidase bei subkutaner Flüssigkeitsgabe nicht zu empfehlen.

Als Injektionsstellen werden die Bauchwand, der Oberschenkel, die Skapularegion, die Axillae und die subklavikuläre Brustwand empfohlen [293]. Die Komplikationsrate ist generell gering. Eine Gewebsschwellung kann durch Reduktion der Infusionsgeschwindigkeit und durch ein Massieren des Gewebes behandelt werden [297]. Im Vergleich zu einer intravenösen Flüssigkeitsgabe werden bei einer subkutanen Flüssigkeitsgabe weniger Kanülen benötigt. Insgesamt waren die Materialkosten bei intravenöser Flüssigkeitsgabe 4-mal so hoch wie bei subkutaner Flüssigkeitsgabe [289]. Die subkutane Flüssigkeitsgabe im häuslichen Bereich oder im Pflegeheim kann Krankenhauseinweisungen vermeiden [289].

\section{Interessenkonflikt}

Gemäß den AWMF-Richtlinien wurden die bestehenden potenziellen Interessenkonflikte zu Beginn der Leitlinienarbeit von allen Autoren bzw. Arbeitsgruppenmitgliedern dargelegt. Die Autoren/Arbeitsgruppenmitglieder haben bei folgenden Punkten entsprechende Angaben gemacht:

Berater- bzw. Gutachtertätigkeit oder Mitglied eines wissenschaftlichen Beirats eines Unternehmens: S. C. Bischoff, J. Arends, F. Dörje, H. Lochs, J. Ockenga, C. Sieber.

Vortragshonorare von Unternehmen: S. C. Bischoff, J. Arends, F. Dörje, D. Volkert, U. Zech, Z. Stanga, H. Lochs, A. Weimann, M. Adolph, J. Ockenga, C. Sieber.

Finanzielle Zuwendungen für Forschungsvorhaben vonseiten eines Unternehmens: S. C. Bischoff, J. Arends, D. Volkert, U. Zech, A. Weimann, C. Sieber.

Besitz von Geschäftsanteilen, Aktien, Fonds mit Beteiligung von Unternehmen: S. Mühlebach.

Die anderen Autoren haben keinen Interessenkonflikt.

Einzelheiten sind im Leitlinienreport hinterlegt. 


\section{Institute}

${ }^{1}$ Universität Hohenheim, Institut für Ernährungsmedizin, Stuttgart, Deutschland

${ }^{2}$ Albert-Ludwigs-Universität, KTB Klinik für Tumorbiologie, Freiburg, Deutschland

${ }^{3}$ Universitätsklinikum Erlangen, Apotheke, Erlangen, Deutschland

${ }^{4}$ Universitätsklinikum Heidelberg, Abteilung Allgemeinmedizin und Versorgungsforschung, Heidelberg, Deutschland

${ }^{5}$ A'LPHA-PHARMA-SERVICE GmbH, Heilbronn, Deutschland

${ }^{6}$ Alexianer Krefeld GmbH, Klinik für Allgemeine Innere Medizin und Altersmedizin, Krefeld, Deutschland

${ }^{7}$ Pharmazentrum, Universität Basel, Department Pharmazeutische Wissenschaften c/o Pharmakoepidemiologie \& Spitalpharmazie, Basel, Schweiz

${ }^{8}$ Medizinische Hochschule Hannover, Klinik für Gastroenterologie, Hepatologie und Endokrinologie, Hannover, Deutschland

${ }^{9}$ Friedrich-Alexander-Universität Erlangen-Nürnberg, Institut für Biomedizin des Alterns, Nürnberg, Deutschland

${ }^{10}$ Universitätsklinikum Heidelberg, Innere Medizin I und Klinische Chemie, Heidelberg, Deutschland

${ }^{11}$ Universitätsspital Bern, Inselspital, Universitätspoliklinik für Endokrinologie, Diabetologie und Klinische Ernährung, Bern, Schweiz

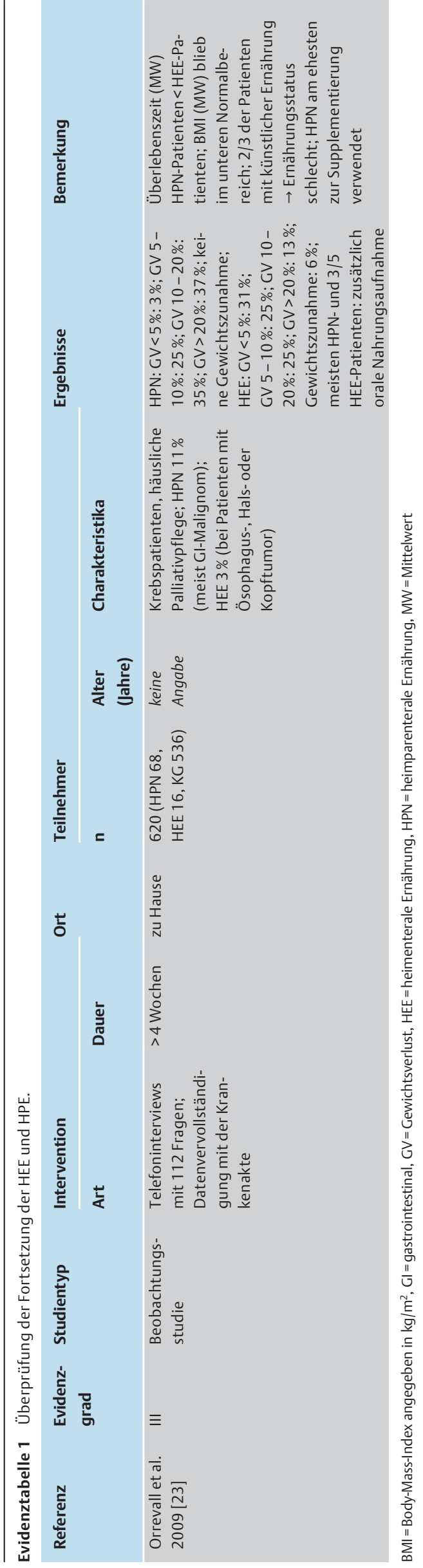




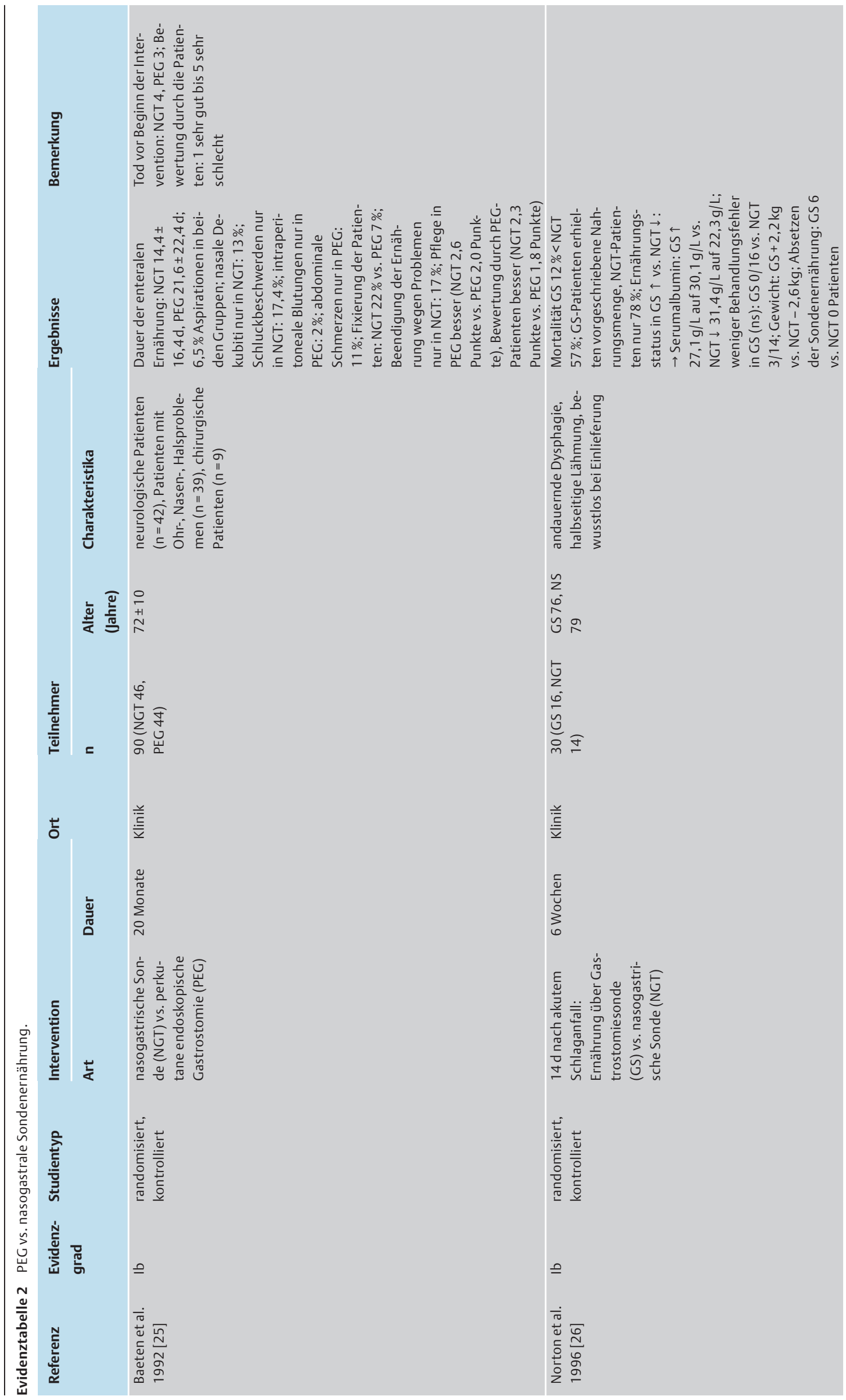




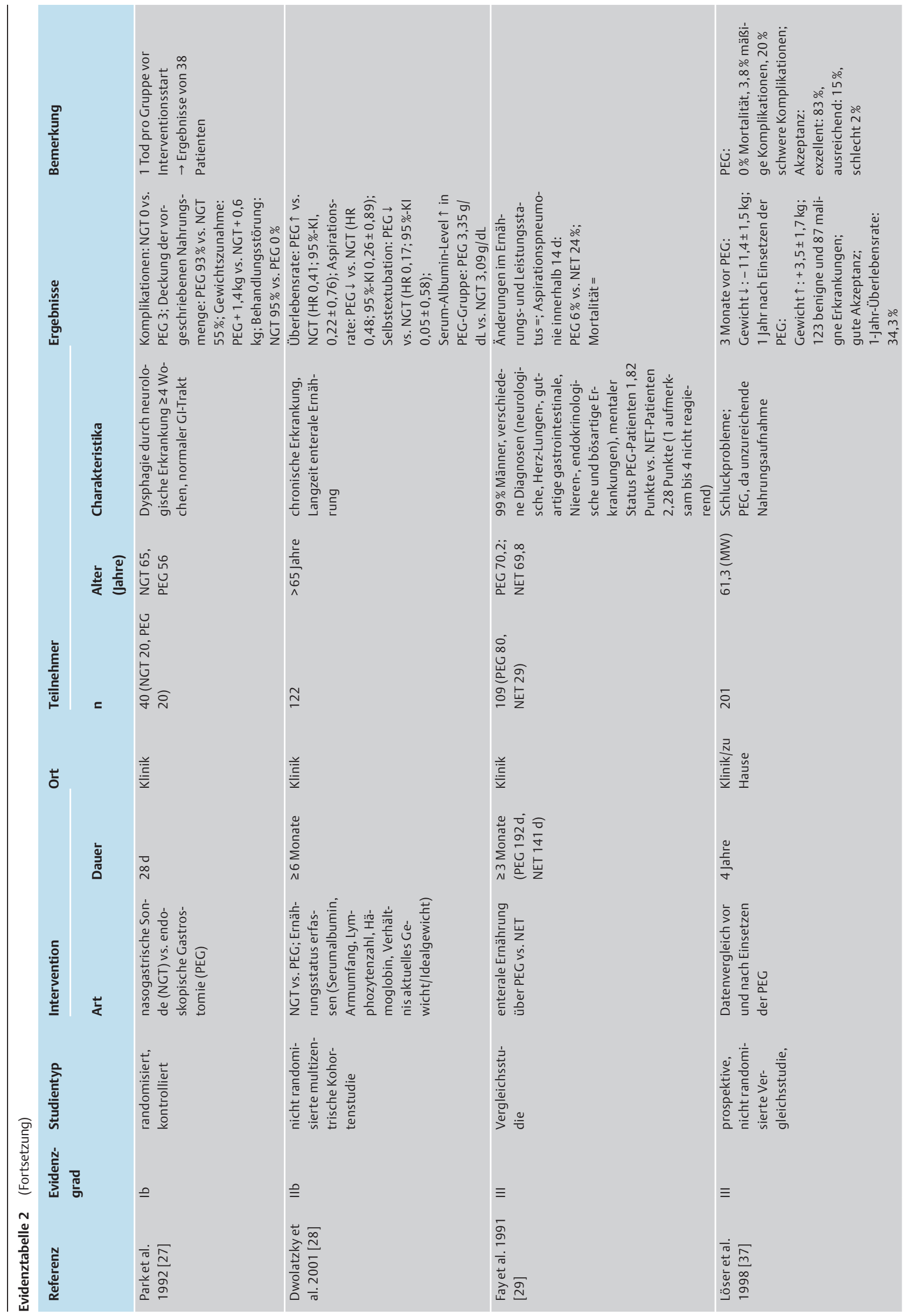




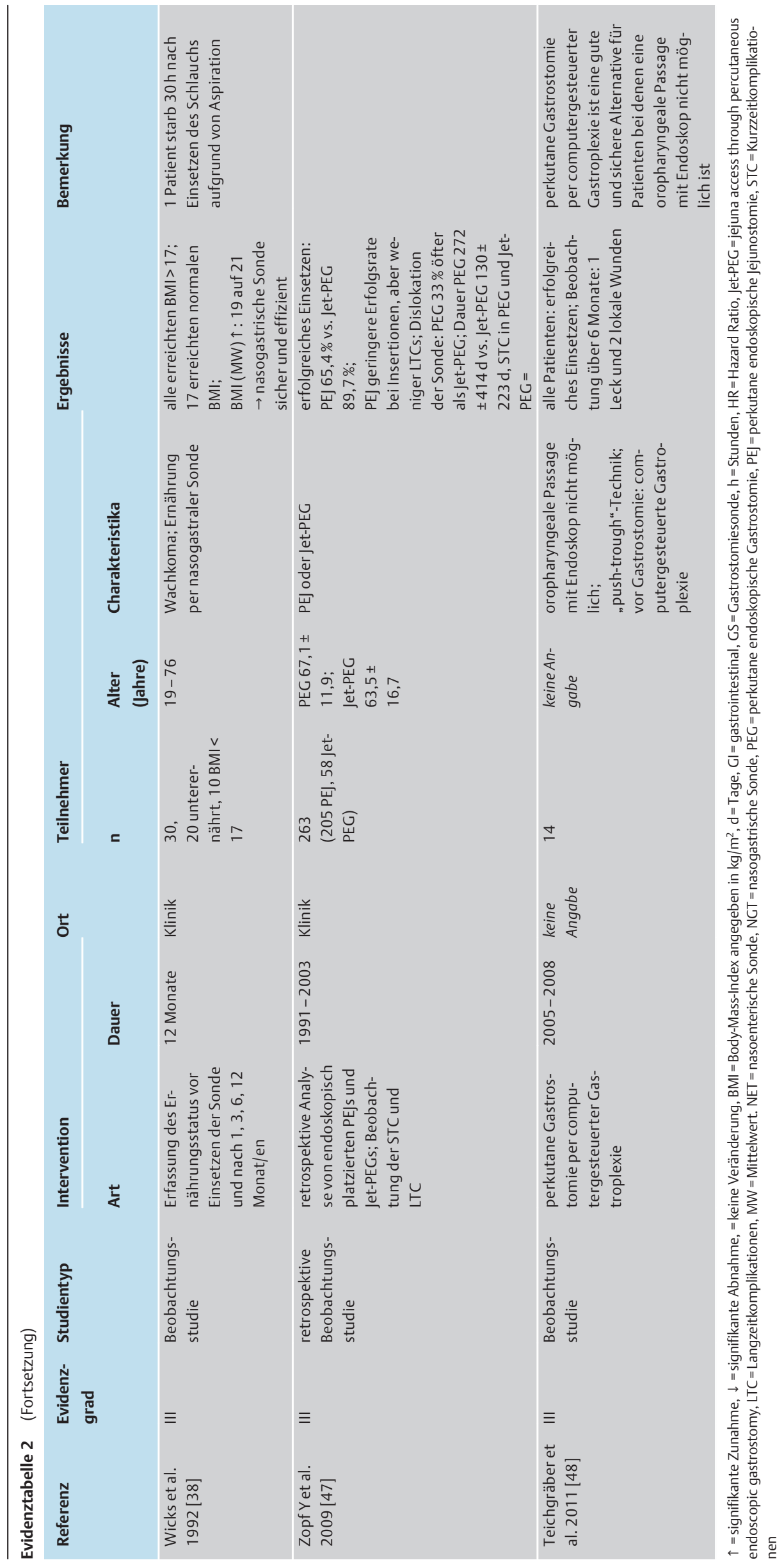




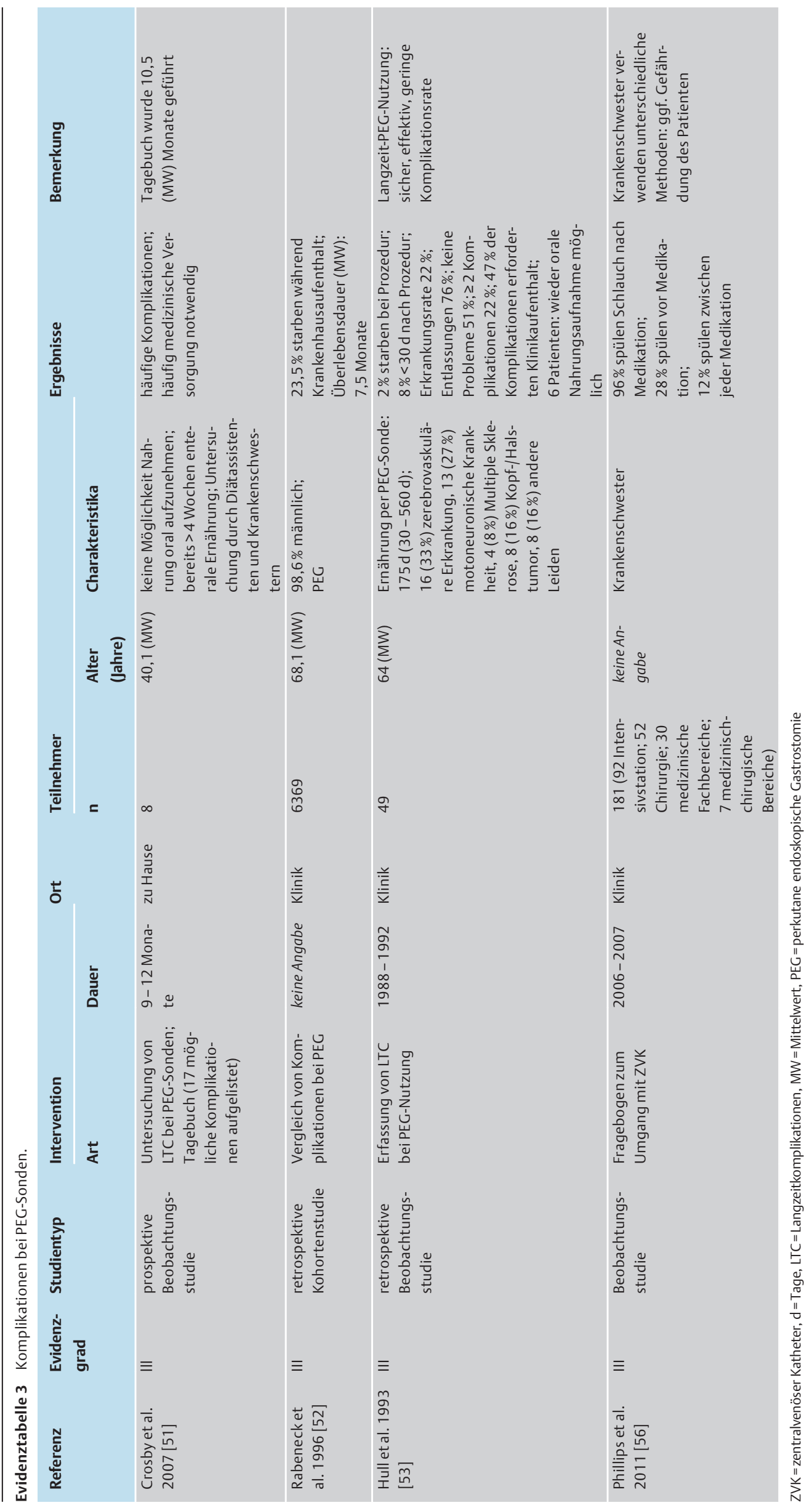




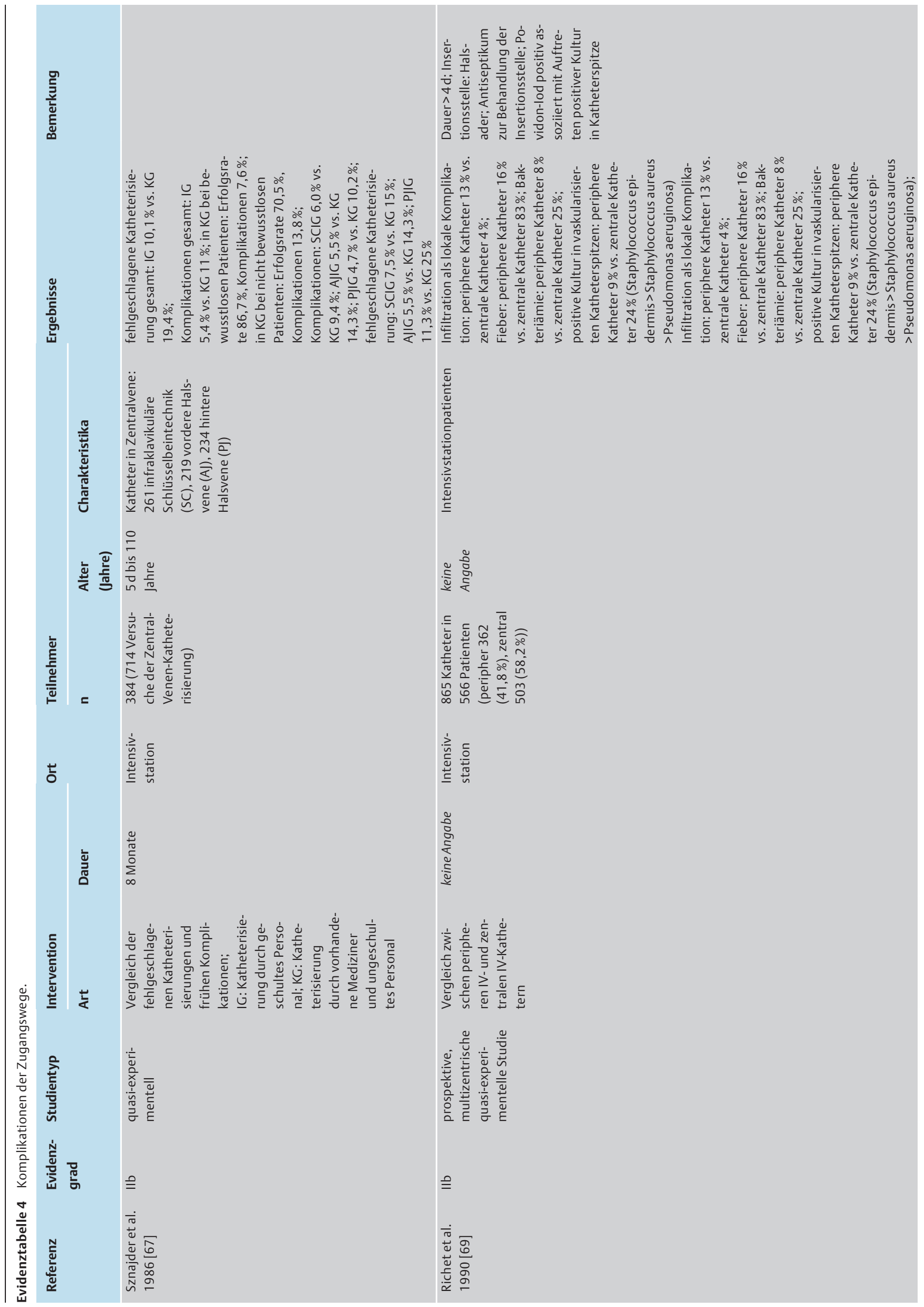




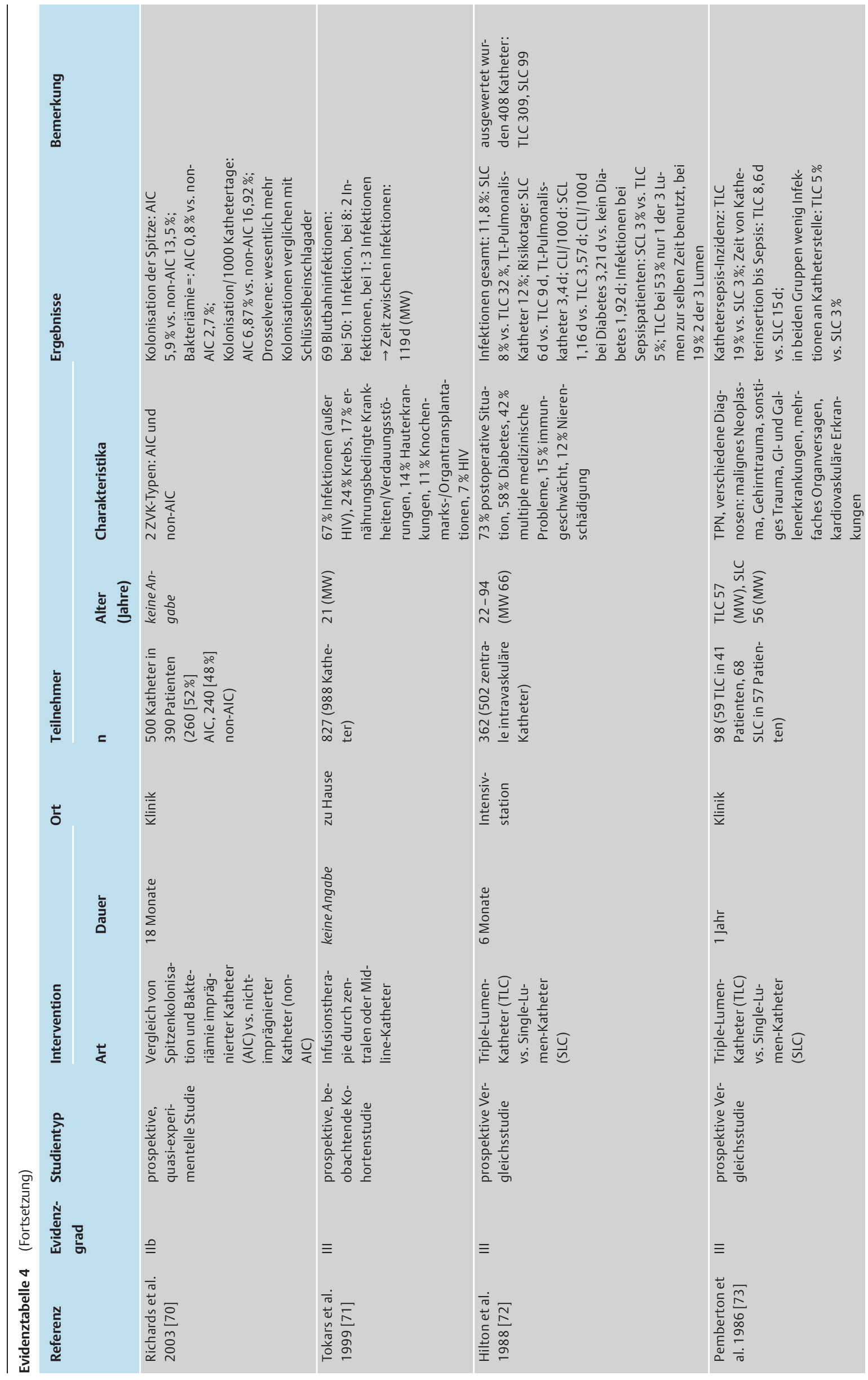




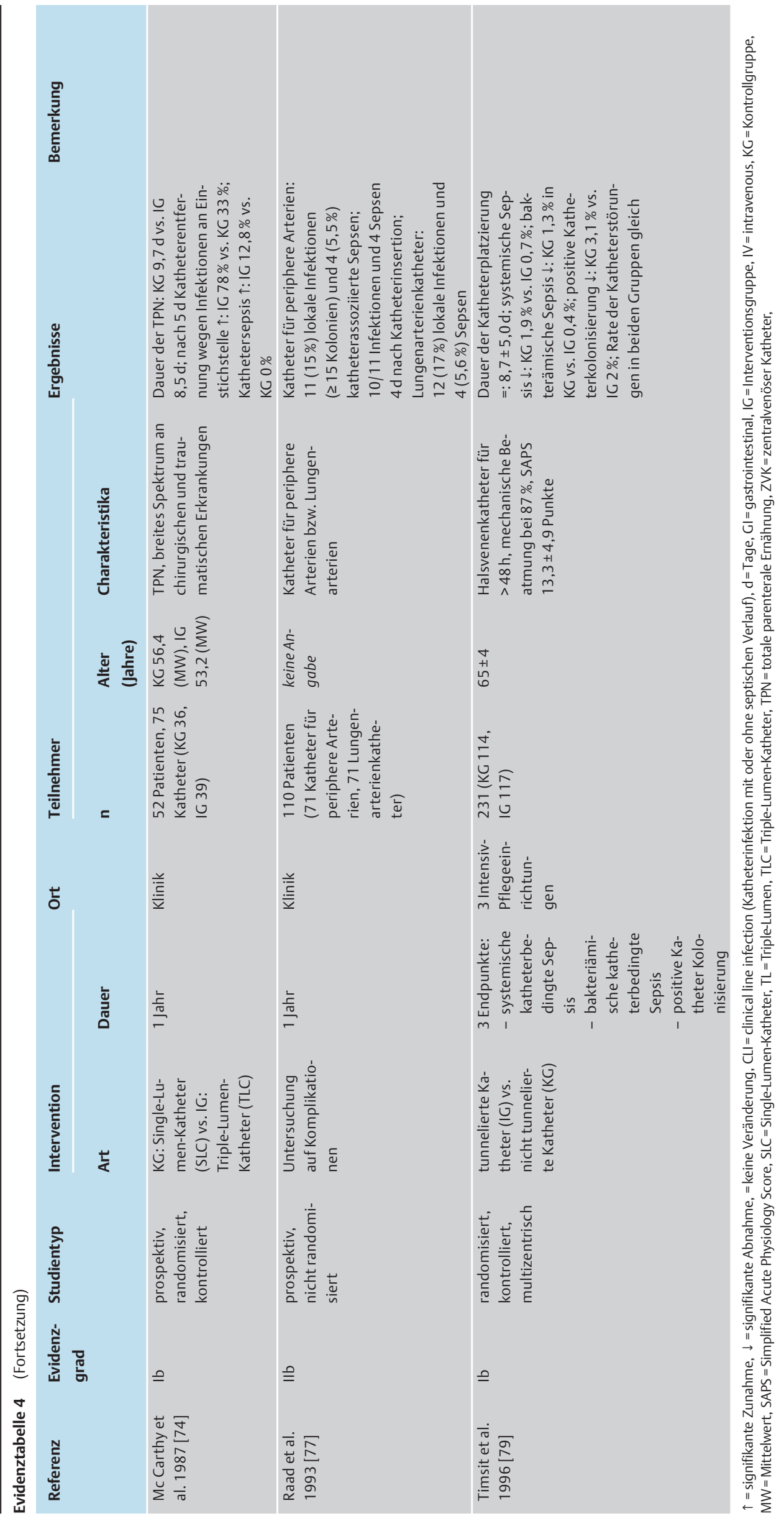




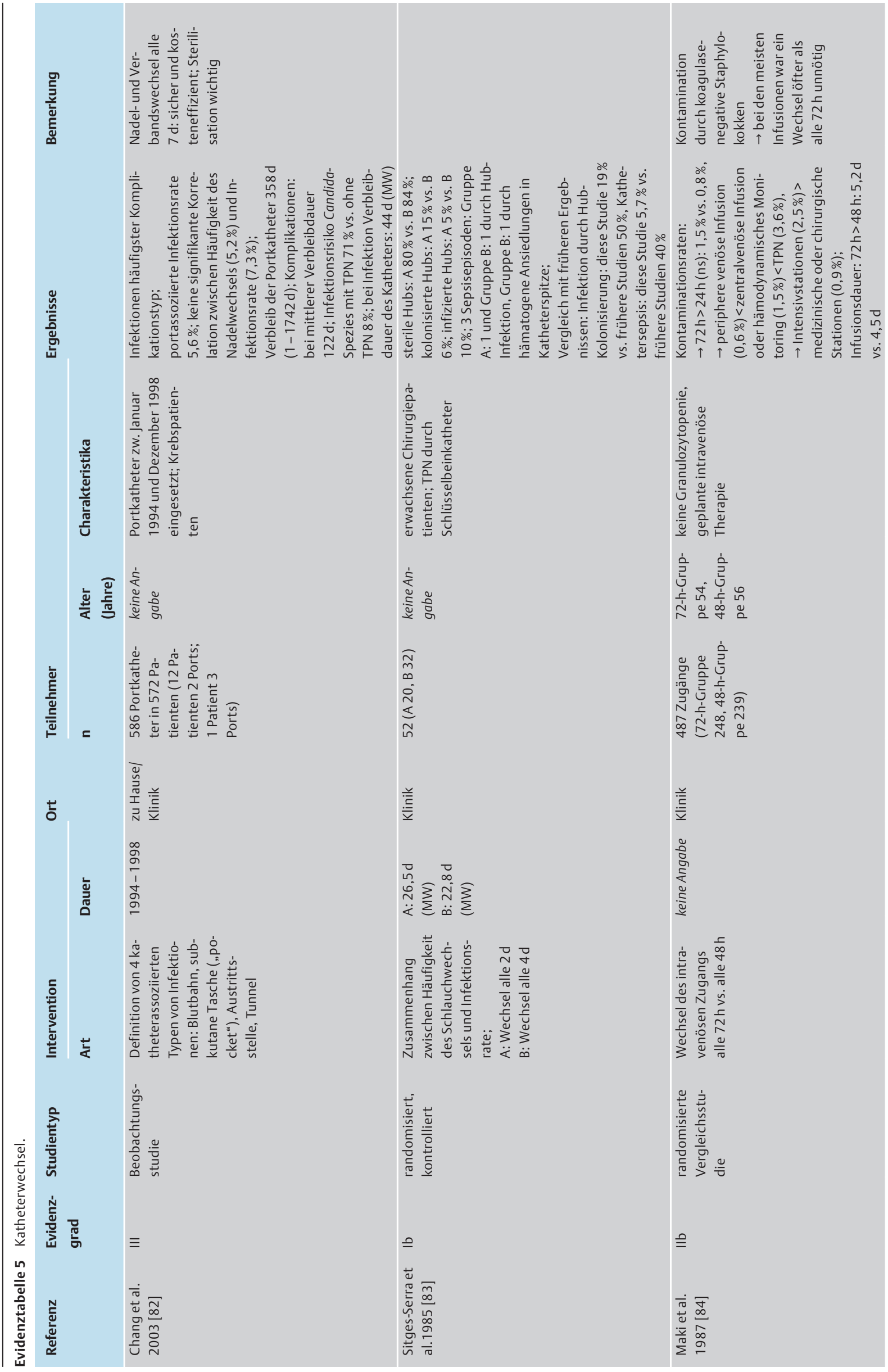



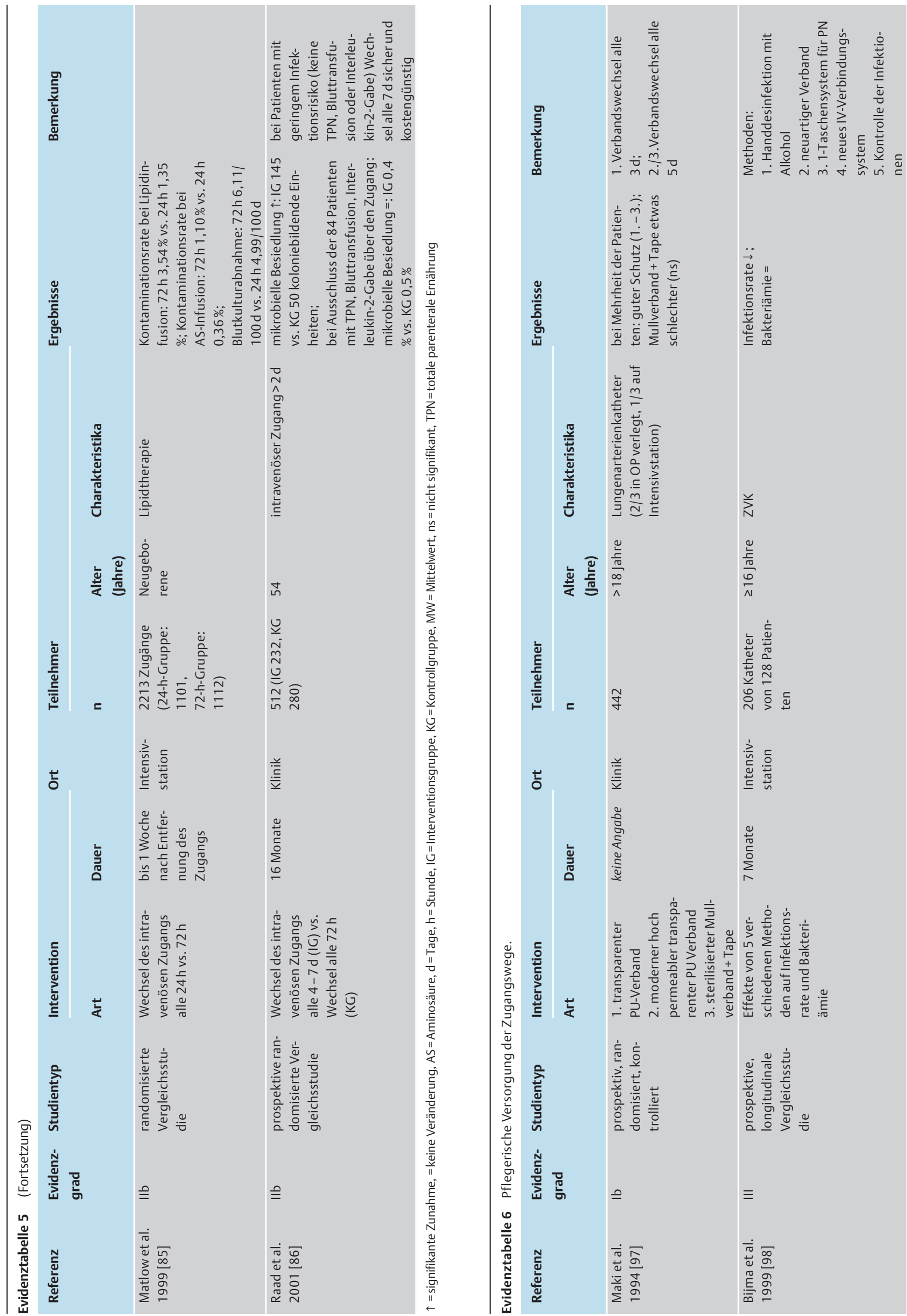


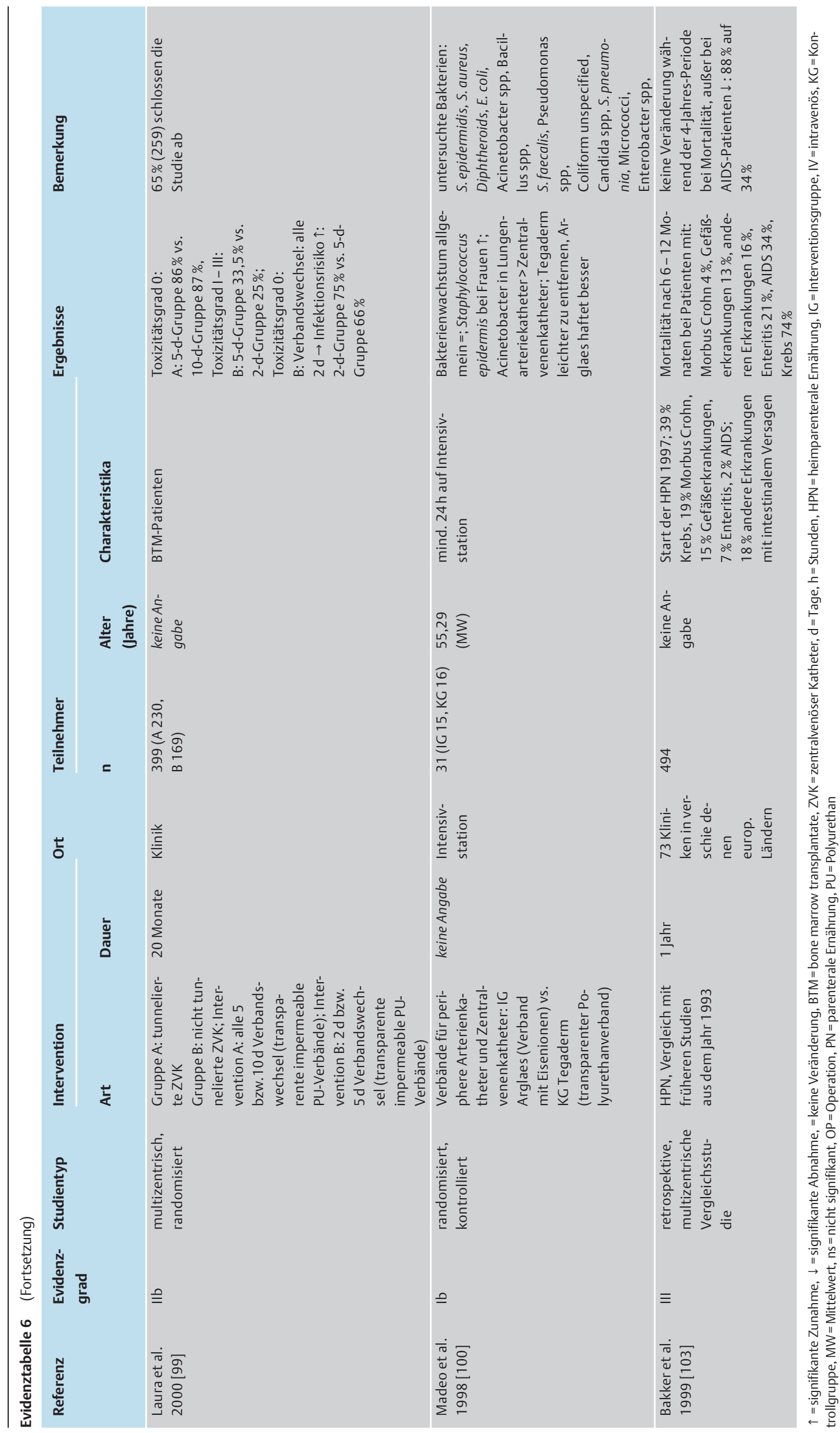




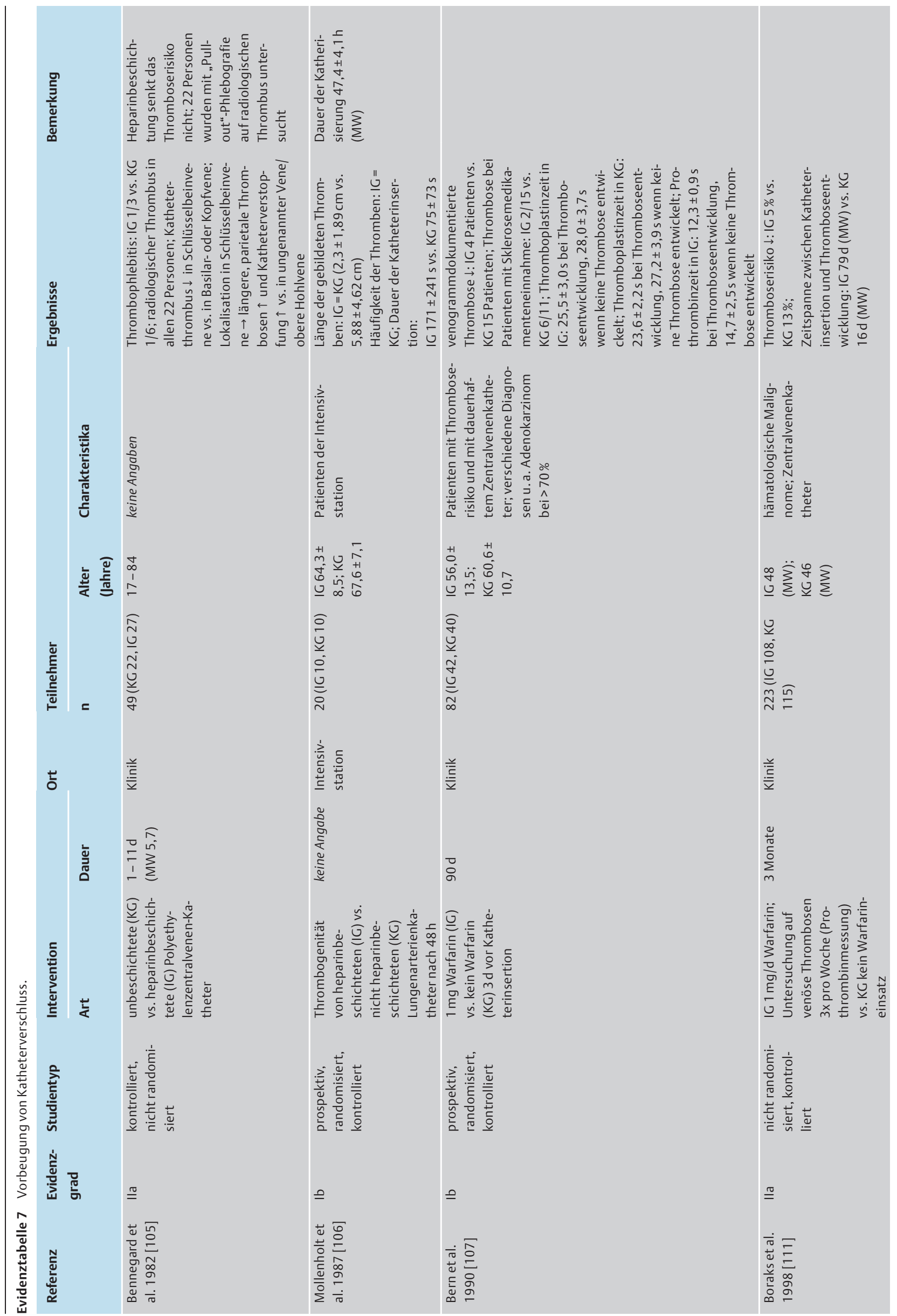




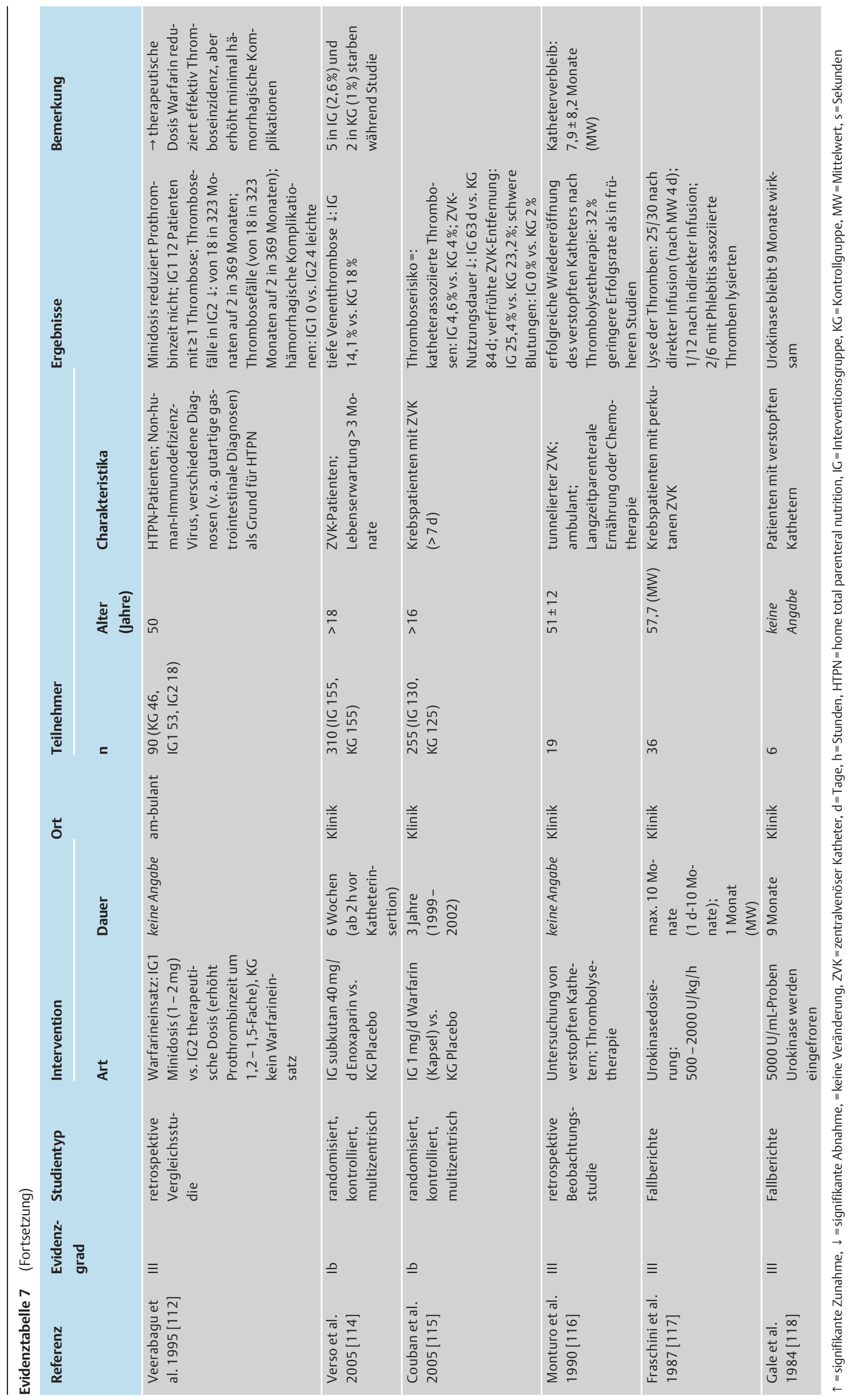




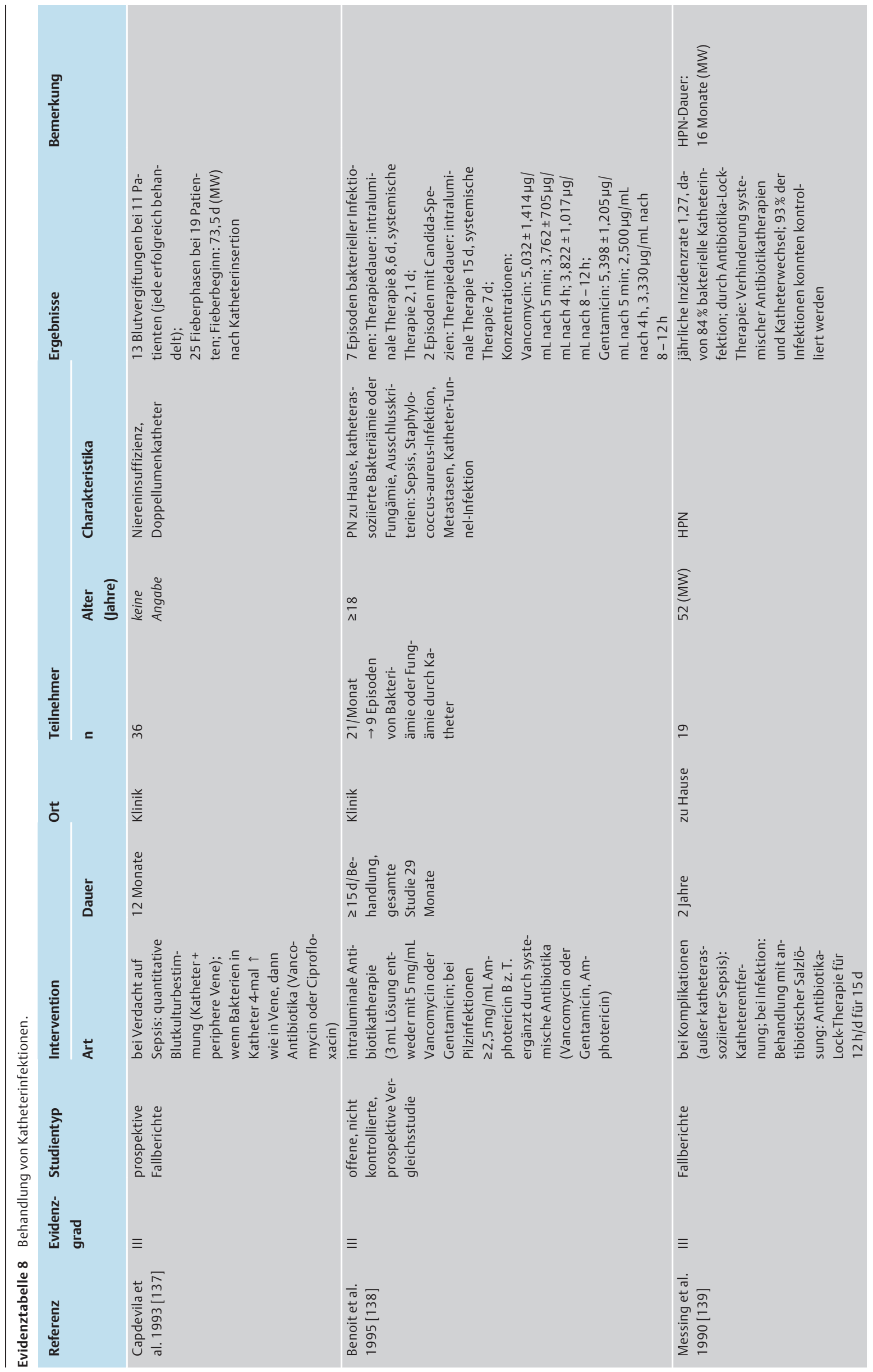




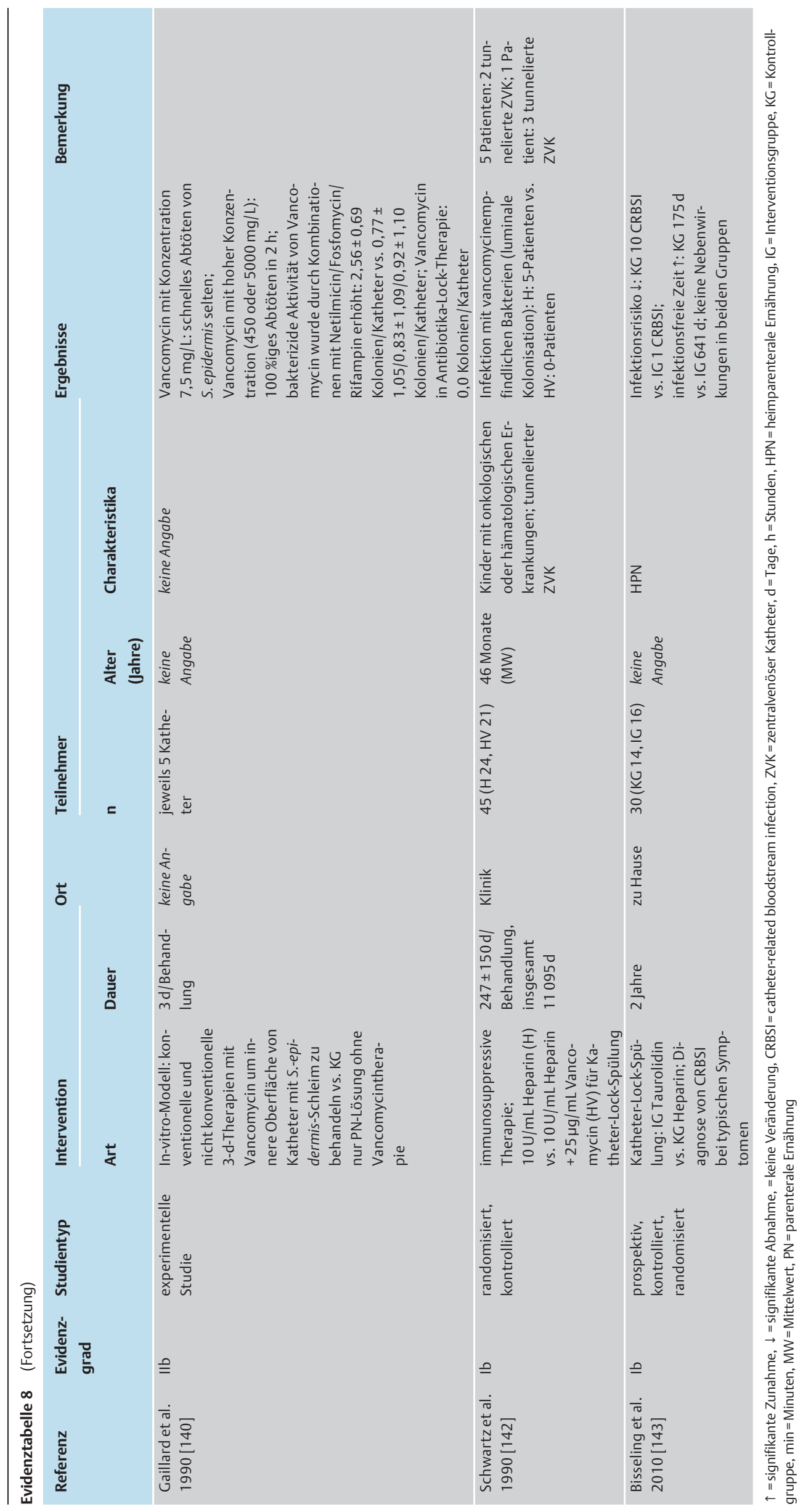




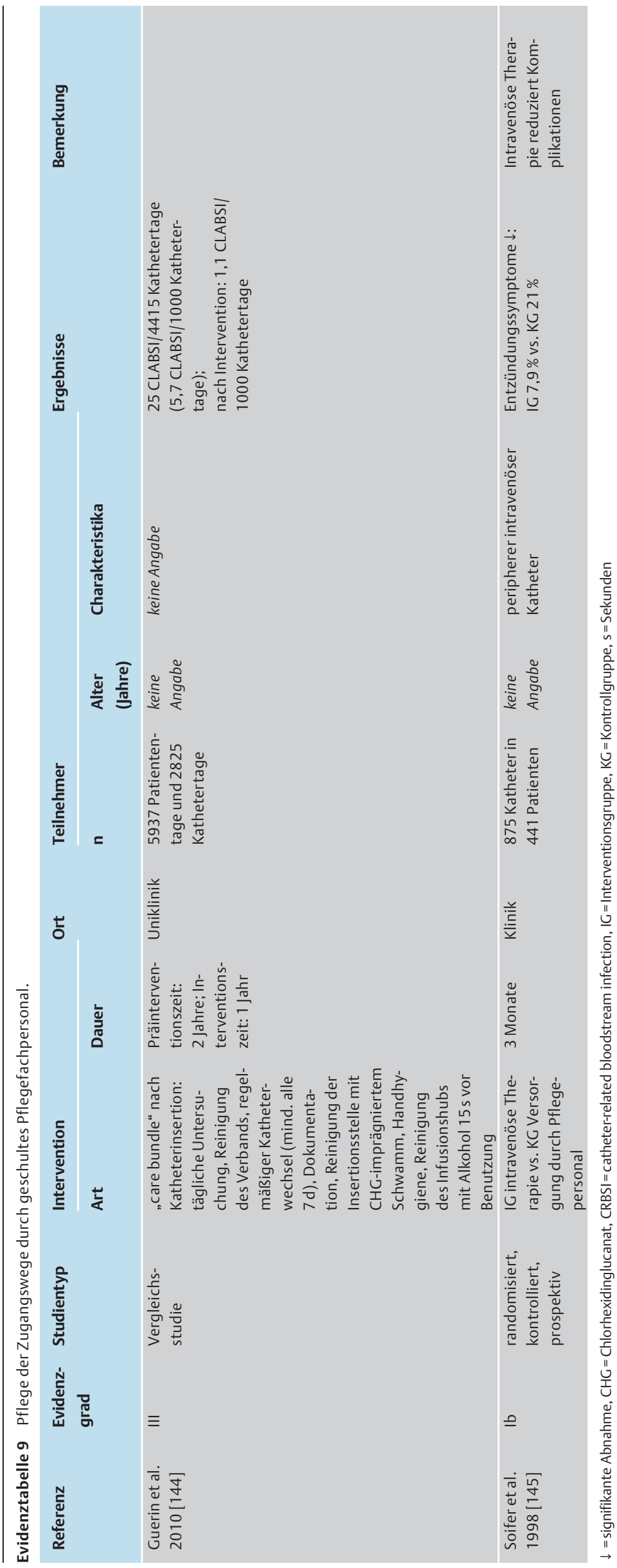



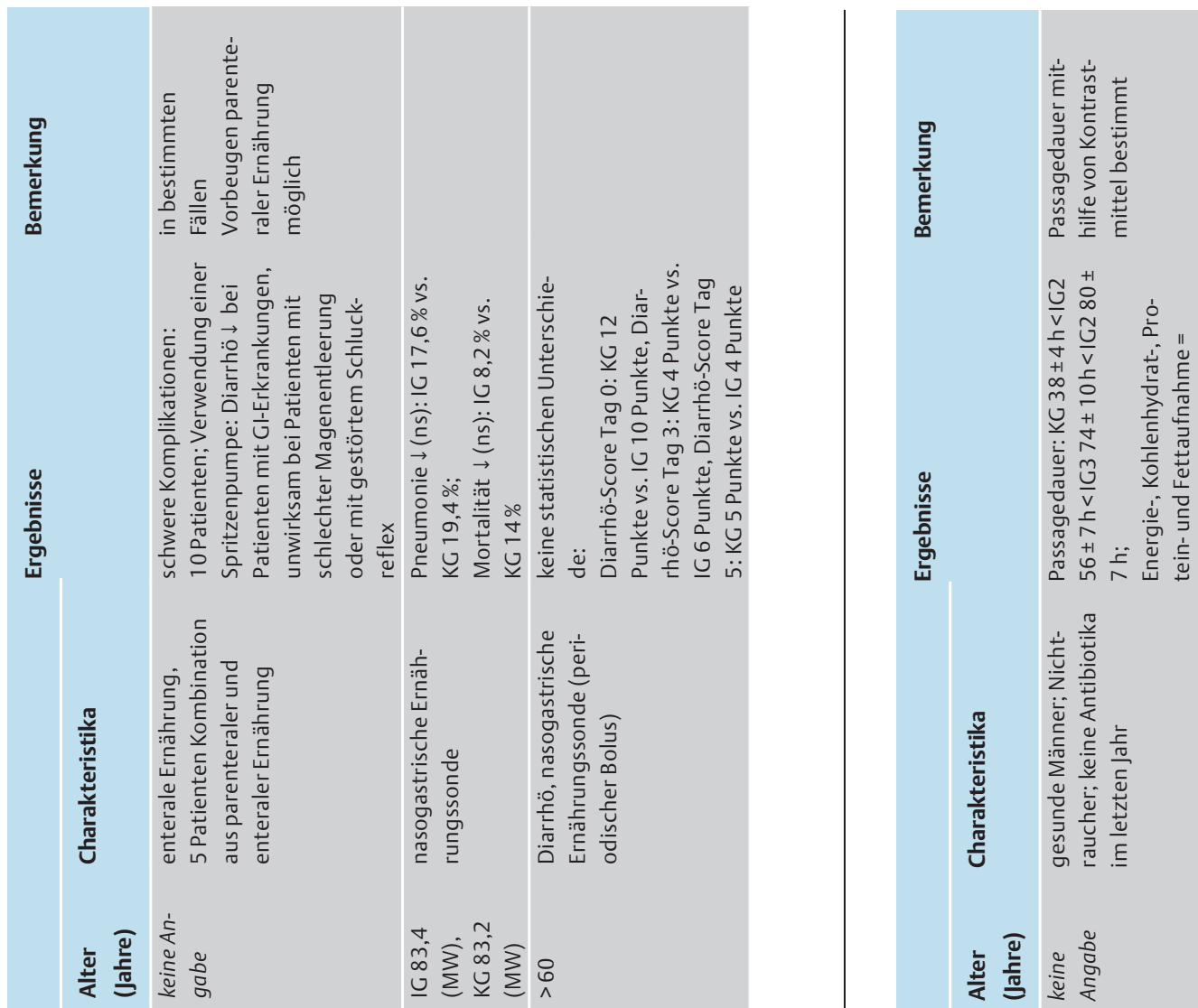

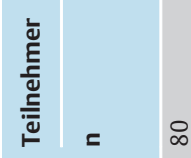

5

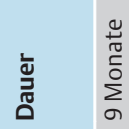

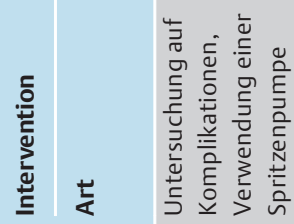

它

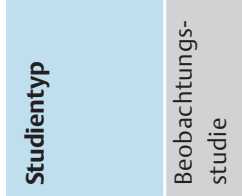

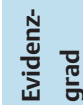

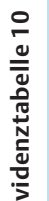

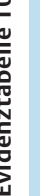

$\equiv$

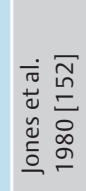

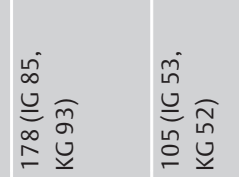

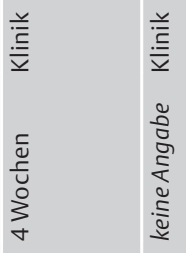

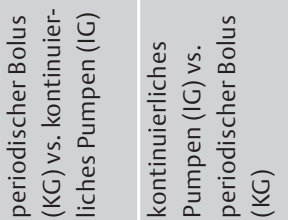

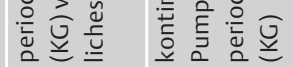
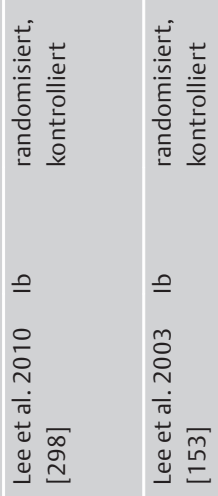

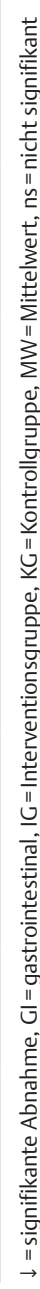

竞
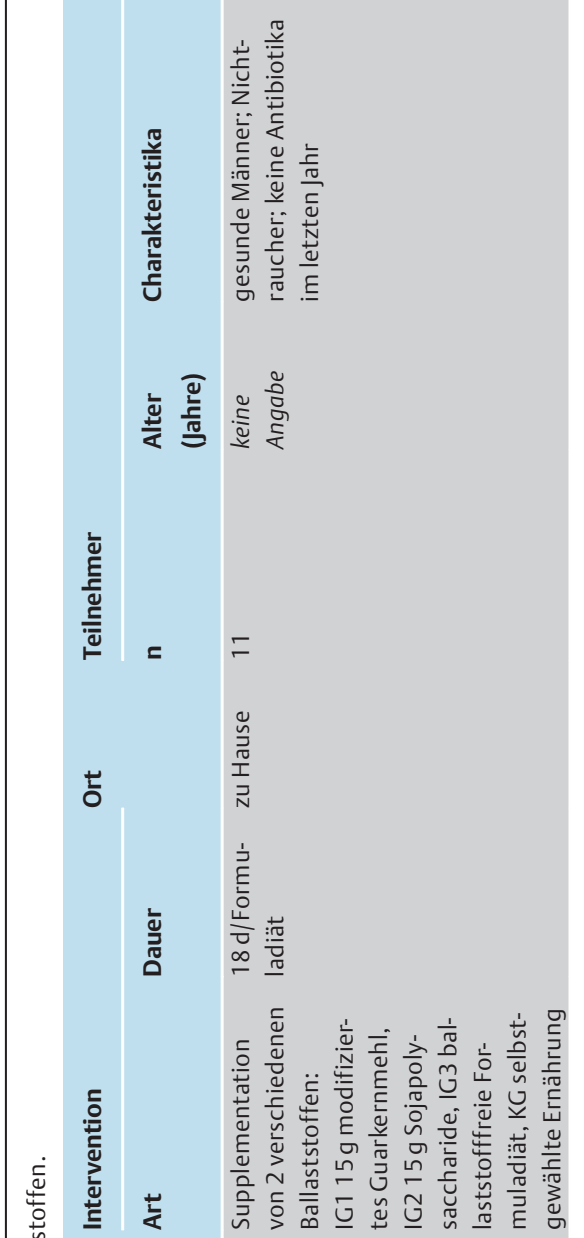

产 

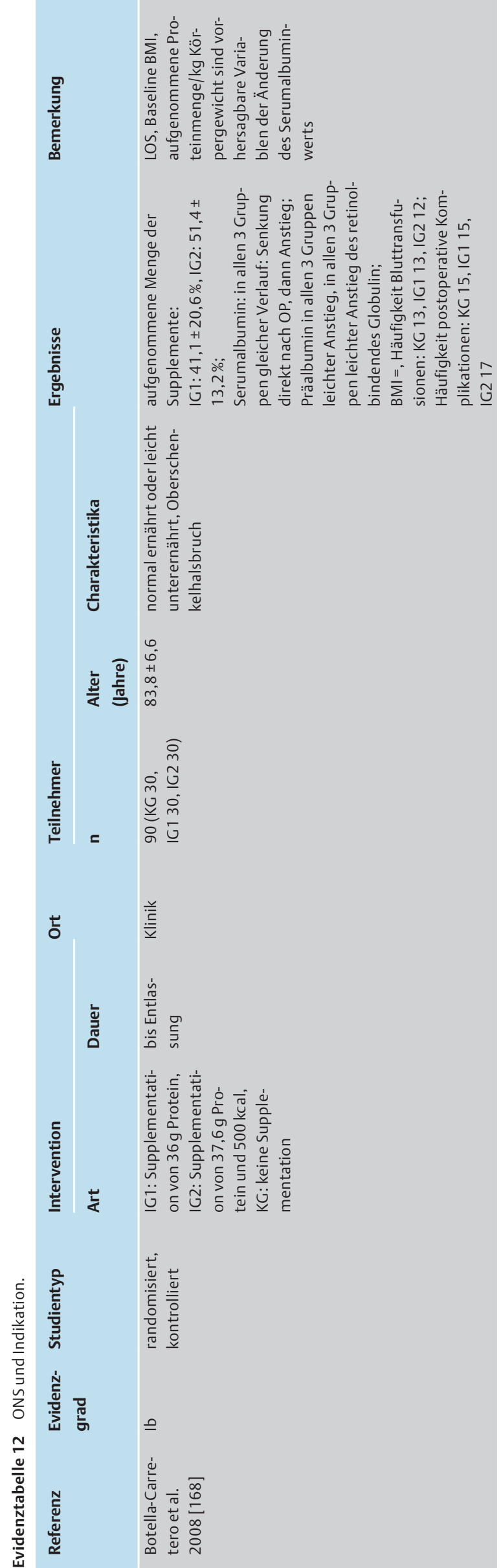

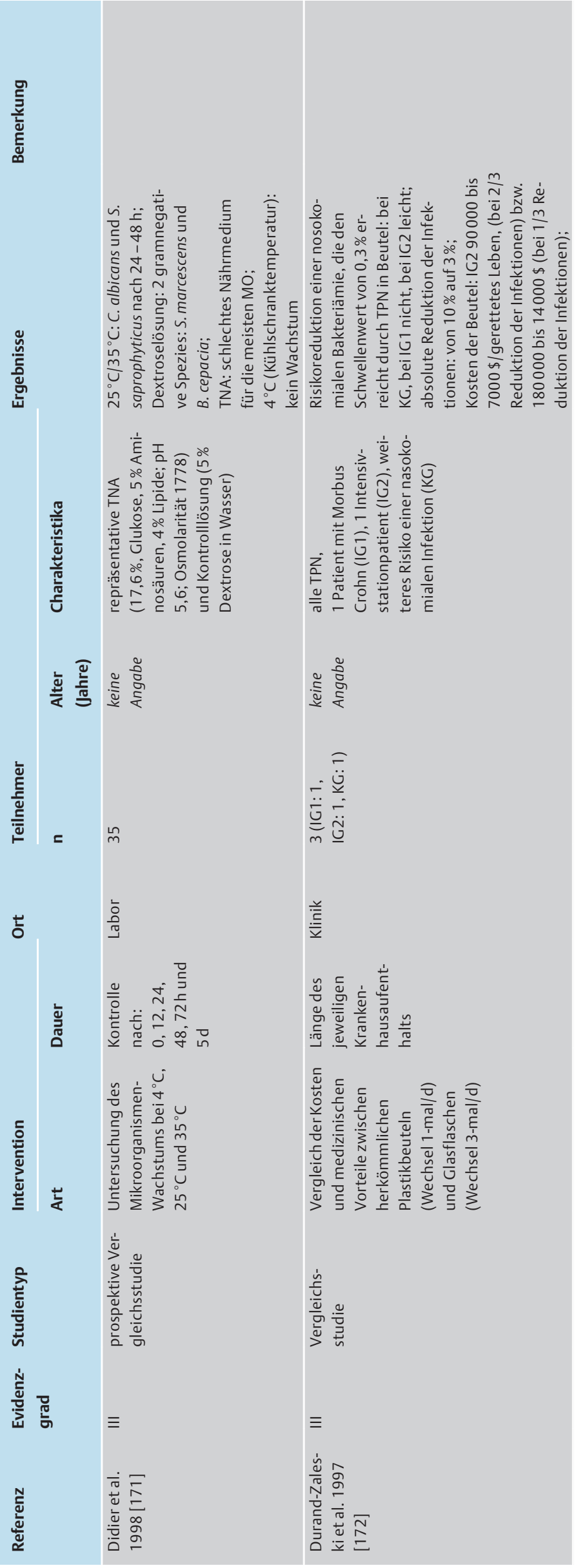




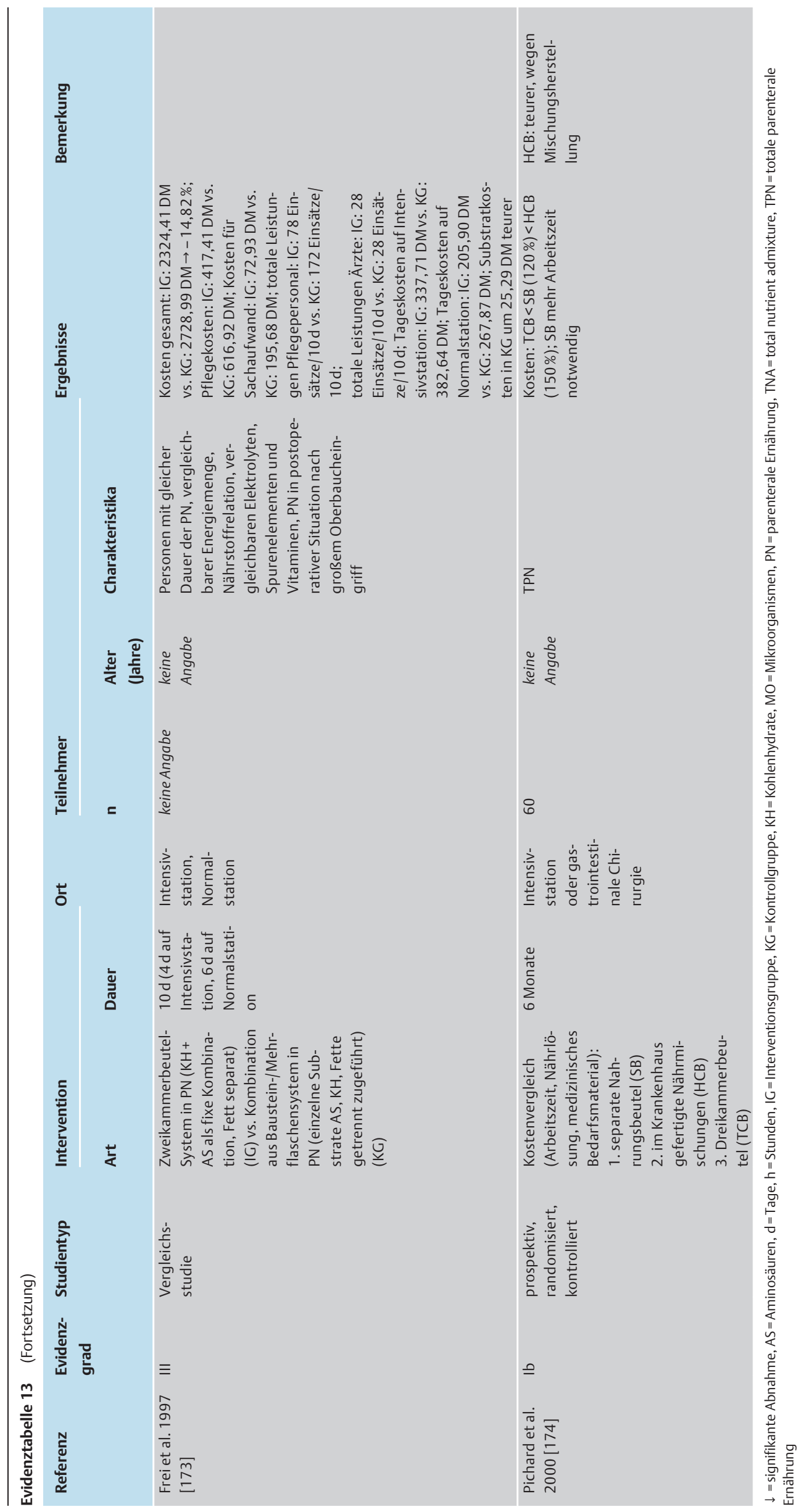



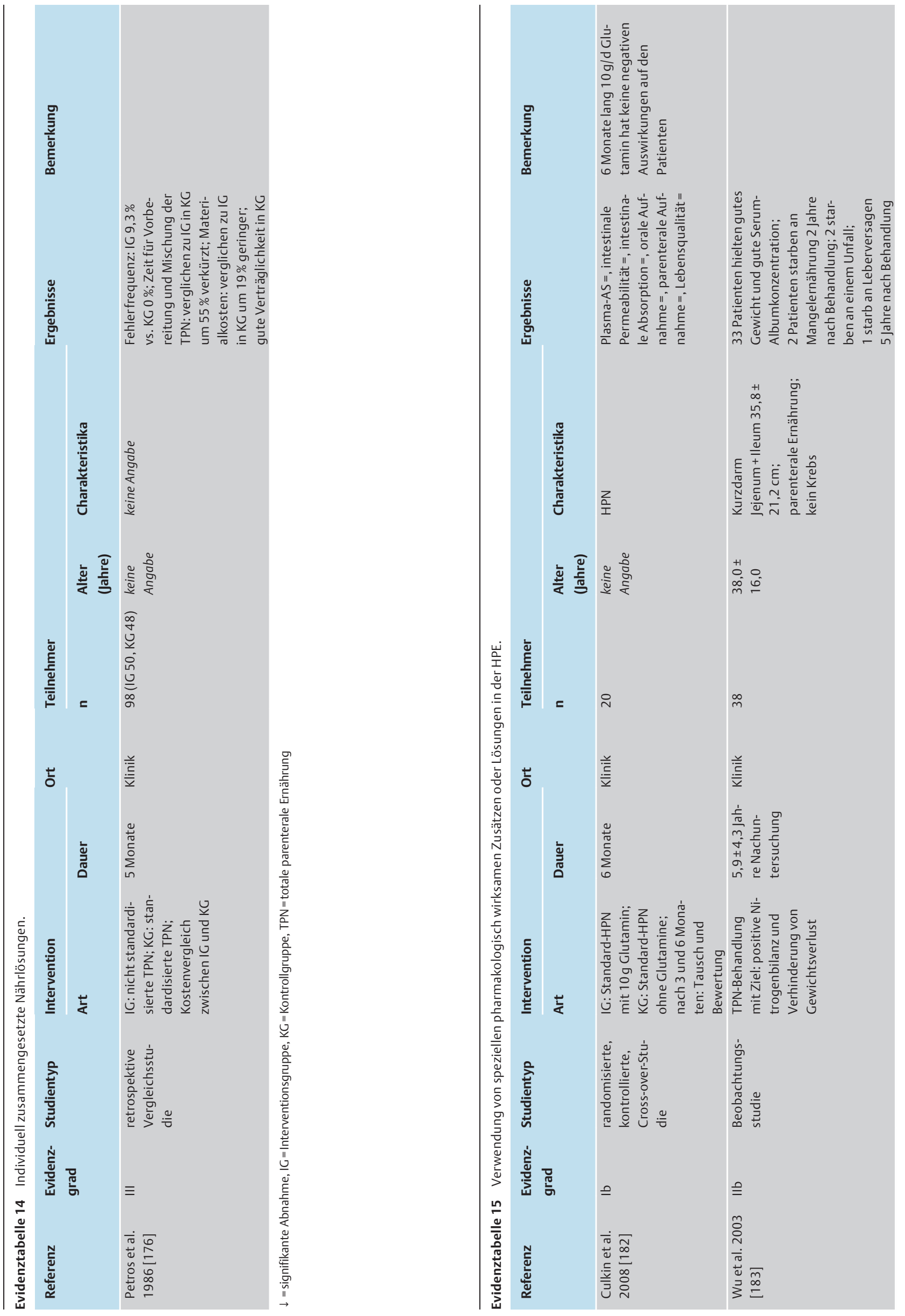


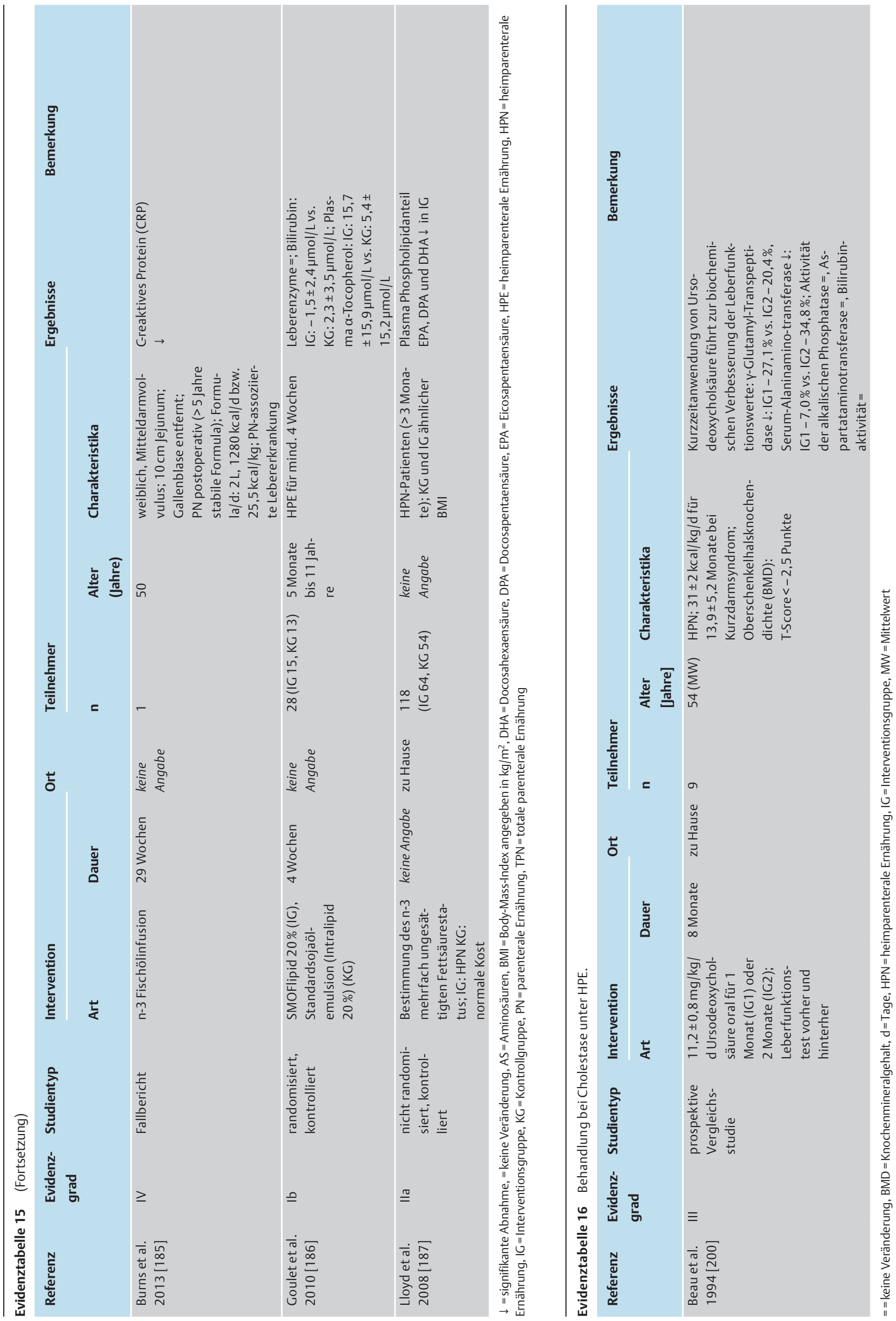




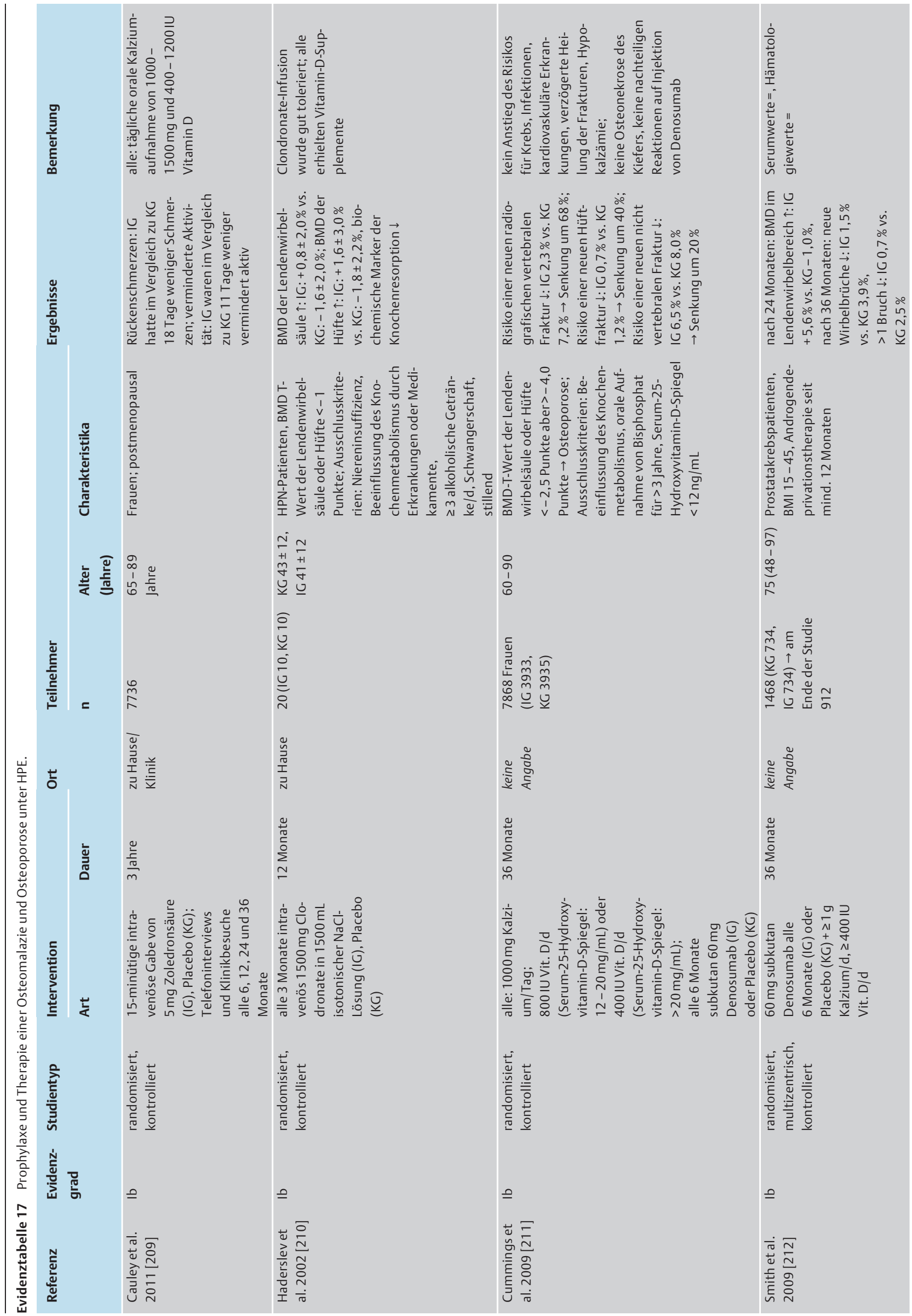




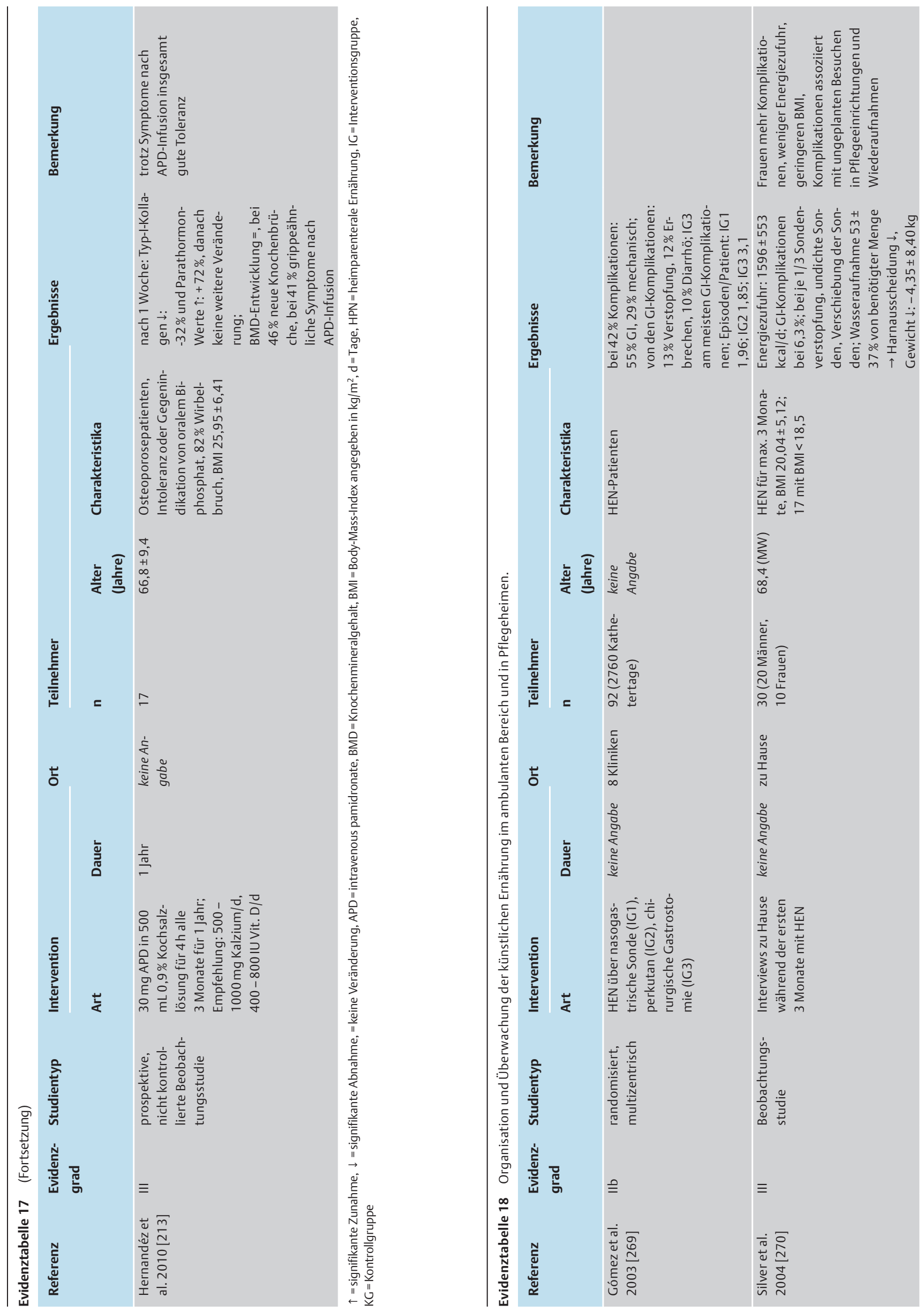




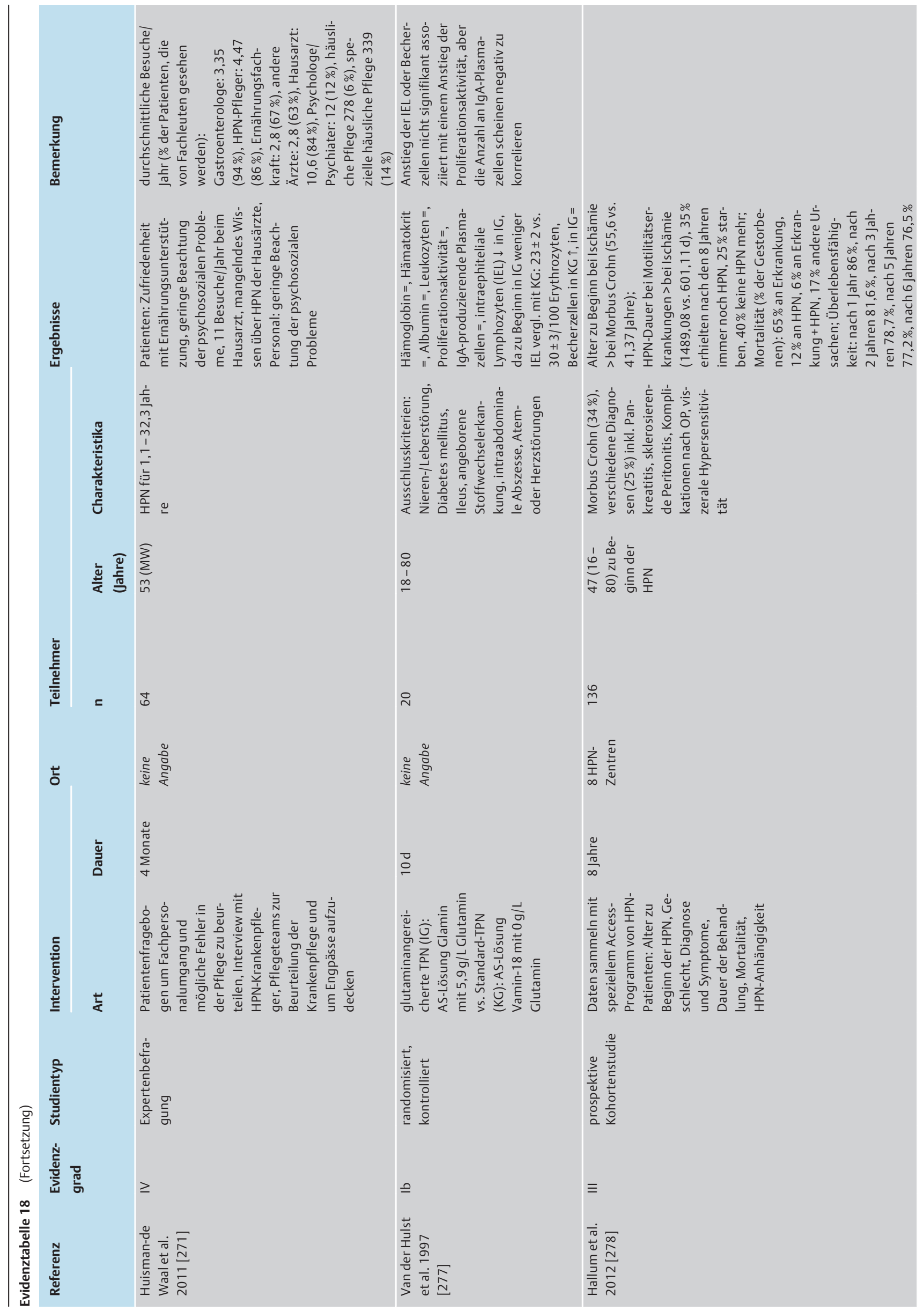




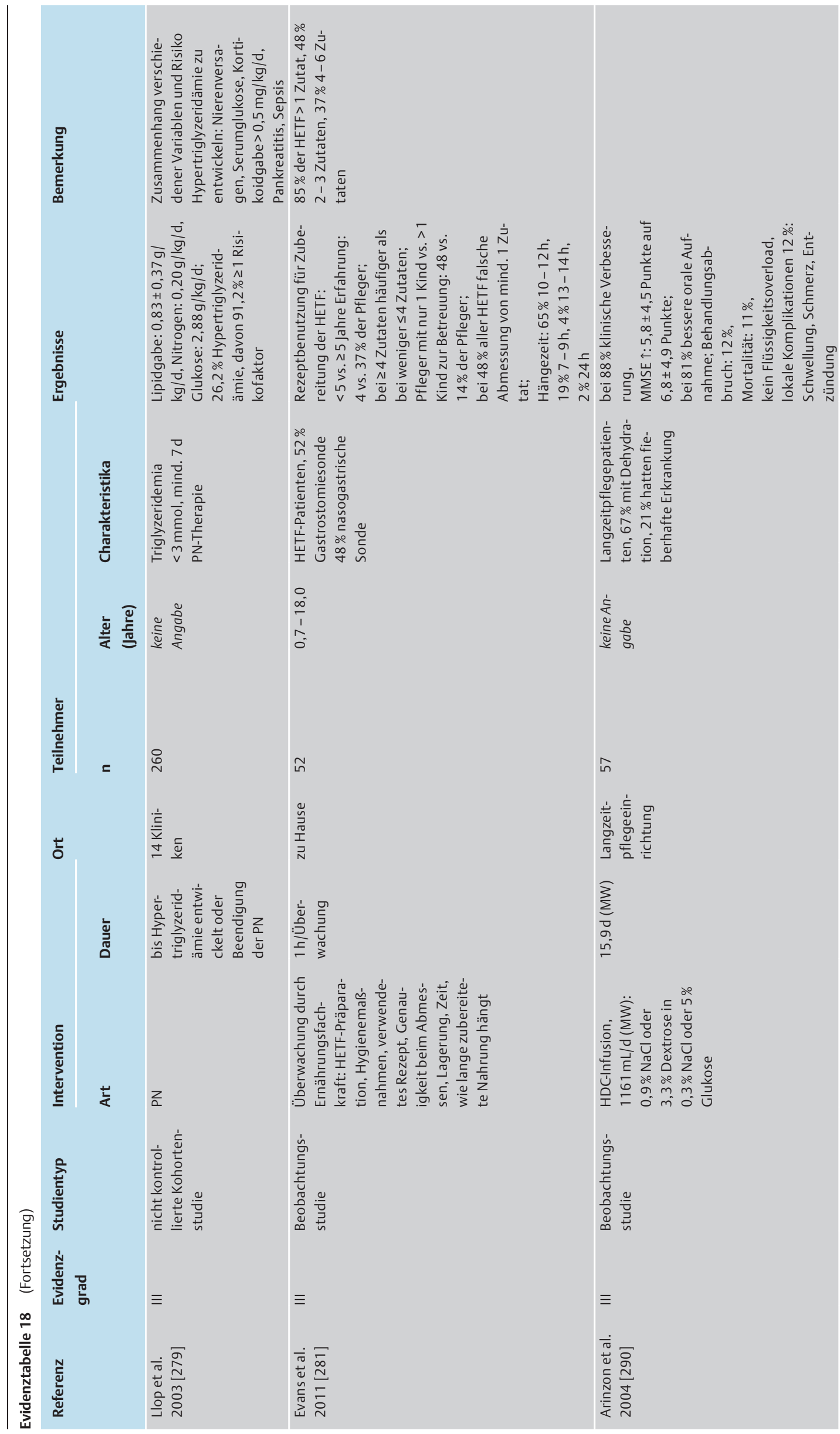




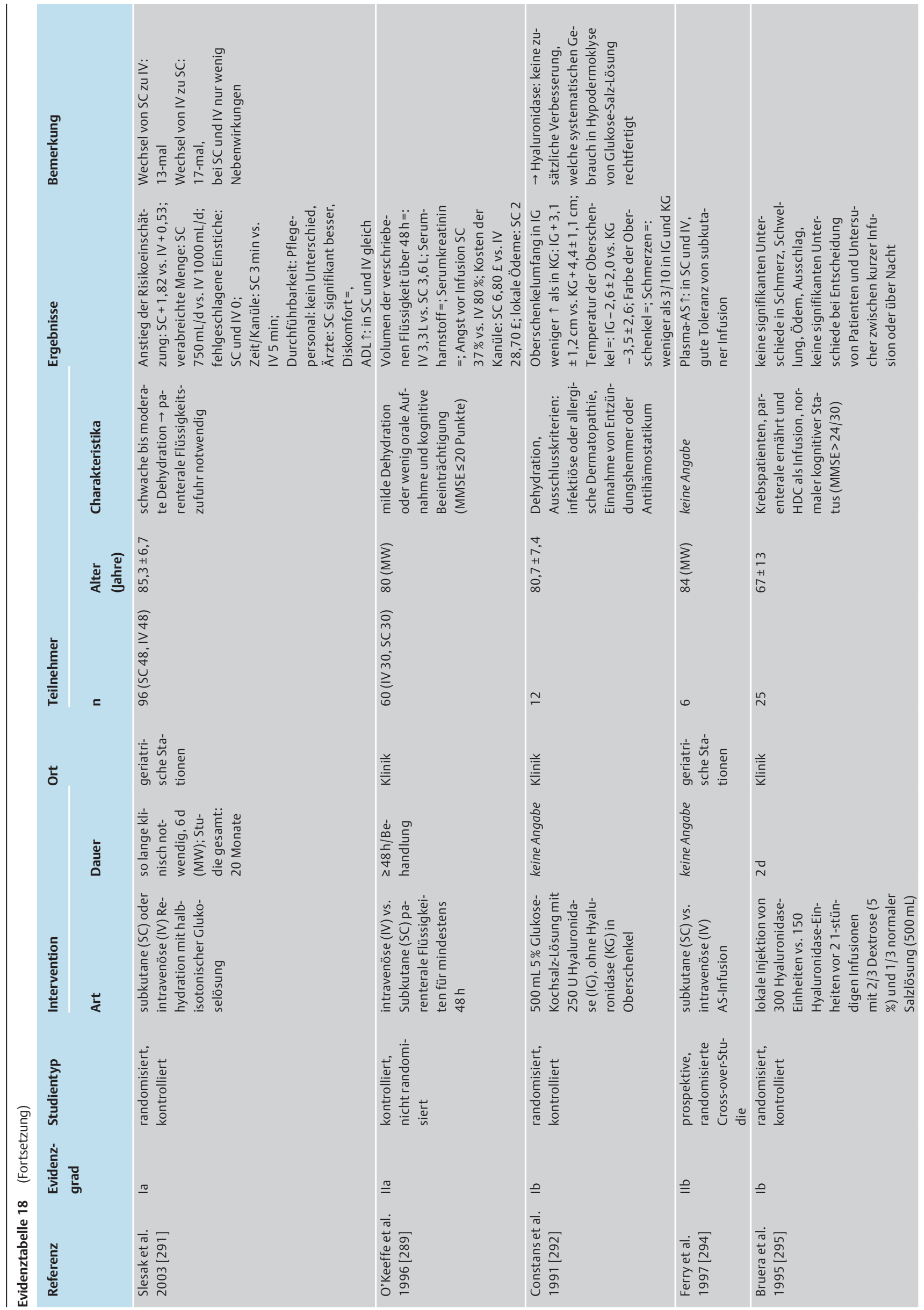




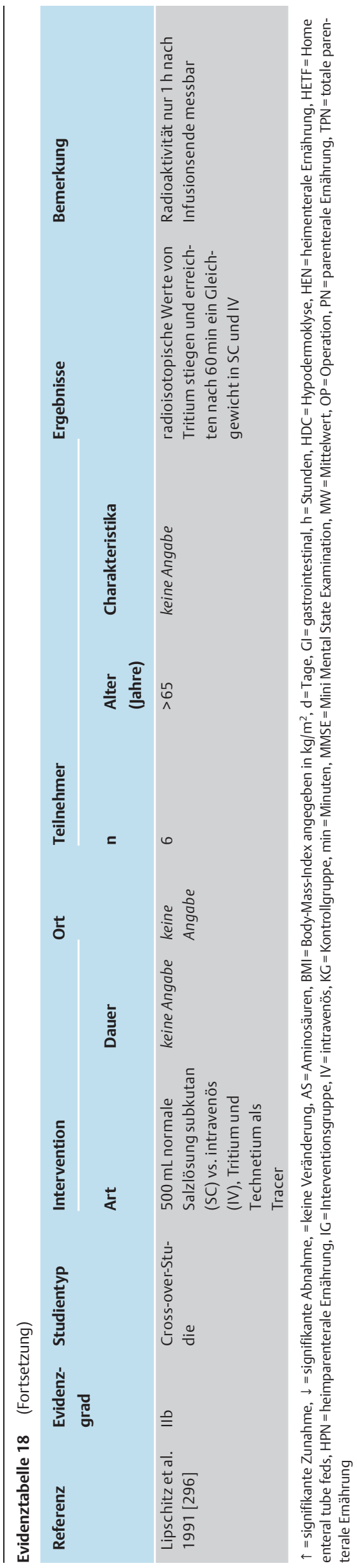

\section{Literatur}

1 Volkert D, Bauer J, Frühwald T et al. Leitlinie der Deutschen Gesellschaft für Ernährungsmedizin (DGEM) in Zusammenarbeit mit der GESKES, der AKE und der DGG: Klinische Ernährung in der Geriatrie - Teil des laufenden S3-Leitlinienprojekts Klinische Ernährung. Aktuel Ernahrungsmed 2013; 38: e1 - e48

2 Wirth R, Dziewas R, Jäger M et al. Leitlinie der Deutschen Gesellschaft für Ernährungsmedizin (DGEM) in Zusammenarbeit mit der GESKES, der AKE, der DGN und der DGG: Klinische Ernährung in der Neurologie - Teil des laufenden S3-Leitlinienprojekts Klinische Ernährung. Aktuel Ernahrungsmed 2013; 38: 257-282

3 Hartl WH, Parhofer KG, Kuppinger D et al. und das DGEM Steering Committee. Leitlinie der Deutschen Gesellschaft für Ernährungsmedizin (DGEM) in Zusammenarbeit mit der GESKES und der AKE: Besonderheiten der Überwachung bei künstlicher Ernährung - Teil des laufenden S3-Leitlinienprojekts Klinische Ernährung. Aktuel Ernahrungsmed 2013; 38: 345-352

4 Oehmichen F, Ballmer PE, Druml C et al. Leitlinie der Deutschen Gesellschaft für Ernährungsmedizin (DGEM) - Ethische und rechtliche Gesichtspunkte der Künstlichen Ernährung. Aktuel Ernahrungsmed 2013; 38: 112 - 117

5 Bischoff SC, Kester L, Meier R et al. Organisation, Verordnung, Zubereitung und Logistik der parenteralen Ernährung im Krankenhaus und zu Hause; die Rolle von Ernährungsteams. Aktuel Ernahrungsmed 2007; 32 (Suppl. 01): S35-S40

6 National Collaborating Centre for Acute Care (UK). Nutrition Support for Adults: Oral Nutrition Support, Enteral Tube Feeding and Parenteral Nutrition. 2006

7 Staun M, Pironi L, Bozzetti F et al. ESPEN Guidelines on Parenteral Nutrition: home parenteral nutrition (HPN) in adult patients. Clin Nutr 2009; 28: 467-479

8 ASPEN Board of Directors and the Clinical Guidelines Task Force. Guidelines for the use of parenteral and enteral nutrition in adult and pediatric patients. JPEN J Parenter Enteral Nutr 2002; 26: 1SA138SA

9 Gillanders L, Angstmann K, Ball P et al. AuSPEN clinical practice guideline for home parenteral nutrition patients in Australia and New Zealand. Nutrition 2008; 24: 998 - 1012

10 Dreesen $M$, Foulon $V$, Vanhaecht $K$ et al. Guidelines recommendations on care of adult patients receiving home parenteral nutrition: a systematic review of global practices. Clin Nutr 2012; 31: 602-608

11 Stingel K, Schütz T, Koller M et al. Leitlinie der Deutschen Gesellschaft für Ernährungsmedizin (DGEM) - Methodik zum Leitlinien-Update Klinische Ernährung. Aktuel Ernahrungsmed 2013; 38: 90 - 96

12 Koller M, Schutz T, Valentini $L$ et al. Outcome models in clinical studies: Implications for designing and evaluating trials in clinical nutrition. Clin Nutr 2013; 32: 650-657

13 Valentini L, Volkert D, Schütz T et al. Leitlinie der Deutschen Gesellschaft für Ernährungsmedizin (DGEM) - DGEM-Terminologie in der Klinischen Ernährung. Aktuel Ernahrungsmed 2013; 38: 97-111

14 Arends J, Bodoky G, Bozzetti $F$ et al. ESPEN Guidelines on Enteral Nutrition: Non-surgical oncology. Clin Nutr 2006; 25: 245-259

15 Bozzetti F, Arends J, Lundholm $K$ et al. ESPEN Guidelines on Parenteral Nutrition: non-surgical oncology. Clin Nutr 2009; 28: 445-454

16 Fearon $K$, Strasser F, Anker SD et al. Definition and classification of cancer cachexia: an international consensus. Lancet Oncol 2011; 12 : 489-495

17 Jensen GL, Mirtallo J, Compher C et al. Adult starvation and diseaserelated malnutrition: a proposal for etiology-based diagnosis in the clinical practice setting from the International Consensus Guideline Committee. JPEN J Parenter Enteral Nutr 2010; 34: 156-159

18 Gripp S, Moeller S, Bolke E et al. Survival prediction in terminally ill cancer patients by clinical estimates, laboratory tests, and self-rated anxiety and depression. J Clin Oncol 2007; 25: 3313-3320

19 Dy SM. Enteral and parenteral nutrition in terminally ill cancer patients: a review of the literature. Am J Hosp Palliat Care 2006; 23 : 369-377

20 Loss J, Eichhorn C, Nagel E. Ethische Aspekte der Ernährungsmedizinischen Versorgung. Aktuel Ernahrungsmed 2004; 29: 329-333

21 Körner U, Biermann E et al. DGEM-Leitlinie Enterale Ernährung: Ethische und rechtliche Gesichtspunkte. Aktuel Ernahrungsmed 2003; 28 (Suppl. 01): S36-S41

22 Rothärmel S, Bischoff SC, Bockenheimer-Lucius G et al. DGEM-Leitlinie Parenterale Ernährung: Ethische und rechtliche Gesichtspunkte. Aktuel Ernahrungsmed 2007; 32 (Suppl. 01): S69-S71 
23 Orrevall $Y$, Tishelman $C$, Permert J et al. The use of artificial nutrition among cancer patients enrolled in palliative home care services. Palliat Med 2009; 23: 556-564

24 Sobotka L, Schneider SM, Berner YN et al. ESPEN Guidelines on Parenteral Nutrition: geriatrics. Clin Nutr 2009; 28: 461 - 466

25 Baeten C, Hoefnagels J. Feeding via nasogastric tube or percutaneous endoscopic gastrostomy. A comparison. Scand J Gastroenterol Suppl 1992; 194: 95 - 98

26 Norton B, Homer-Ward M, Donnelly MT et al. A randomised prospective comparison of percutaneous endoscopic gastrostomy and nasogastric tube feeding after acute dysphagic stroke. BMJ 1996; 312: $13-16$

27 Park RH, Allison MC, Lang J et al. Randomised comparison of percutaneous endoscopic gastrostomy and nasogastric tube feeding in patients with persisting neurological dysphagia. BMJ 1992; 304: $1406-1409$

28 Dwolatzky T, Berezovski S, Friedmann $R$ et al. A prospective comparison of the use of nasogastric and percutaneous endoscopic gastrostomy tubes for long-term enteral feeding in older people. Clin Nutr $2001 ; 20: 535-540$

29 Fay DE, Poplausky $M$, Gruber $M$ et al. Long-term enteral feeding: a retrospective comparison of delivery via percutaneous endoscopic gastrostomy and nasoenteric tubes. Am J Gastroenterol 1991; 86: $1604-1609$

30 Carver $A D$, Dobson AM. Effects of dietary supplementation of elderly demented hospital residents. Journal of Human Nutrition and Dietetics 1995; 8: 389 - 394

31 Gomes CA Jr, Lustosa SA, Matos D et al. Percutaneous endoscopic gastrostomy versus nasogastric tube feeding for adults with swallowing disturbances. Cochrane Database Syst Rev 2010:(11): CD008096 doi: CD008096

32 Loser C, Aschl G, Hebuterne X et al. ESPEN guidelines on artificial enteral nutrition - percutaneous endoscopic gastrostomy (PEG). Clin Nutr 2005; 24: 848-861

33 Bell SD, Carmody EA, Yeung EY et al. Percutaneous gastrostomy and gastrojejunostomy: additional experience in 519 procedures. Radiology $1995 ; 194: 817-820$

34 Sarr MG. Appropriate use, complications and advantages demonstrated in 500 consecutive needle catheter jejunostomies. Br J Surg 1999; 86: $557-561$

35 Shike M, Latkany L, Gerdes $H$ et al. Direct percutaneous endoscopic jejunostomies for enteral feeding. Gastrointest Endosc 1996; 44: 536- 540

36 Maple JT, Petersen BT, Baron TH et al. Direct percutaneous endoscopic jejunostomy: outcomes in 307 consecutive attempts. Am J Gastroenterol 2005; 100: $2681-2688$

37 Loser C, Wolters S, Folsch UR. Enteral long-term nutrition via percutaneous endoscopic gastrostomy (PEG) in 210 patients: a four-year prospective study. Dig Dis Sci 1998; 43: 2549 - 2557

38 Wicks C, Gimson A, Vlavianos $P$ et al. Assessment of the percutaneous endoscopic gastrostomy feeding tube as part of an integrated approach to enteral feeding. Gut 1992; 33: 613-616

39 Slater $R$. Percutaneous endoscopic gastrostomy feeding: indications and management. Br J Nurs 2009; 18: 1036-1043

40 Eberlein $T$, Fendler $H$, Rother $A$ et al. Versorgung und Pflege der Eintrittsstellen von PEG/PEJ-Sonden. Die Schwester Der Pfleger 2007; 46: $38-42$

41 Aschl G, Kirchgatterer A, Fleischer $M$ et al. The frequency of wound infections after PEG-placement and utilization of glycogel wound dressing: a randomized controlled trial. Wien Klin Wochenschr 2008 ; 120: $224-227$

42 Judges D, Beverly S, Rio A et al. Clinical guidelines and enteral nutrition support: a survey of dietetic practice in the United Kingdom. Eur J Clin Nutr 2012; 66: $130-135$

43 Kenny DJ, Goodman P. Care of the patient with enteral tube feeding: an evidence-based practice protocol. Nurs Res 2010; 59: 22 - 31

44 Löser C, Keymling M. Praxis der Enteralen Ernährung: Indikationen, Technik, Nachsorge. Stuttgart: Georg Thieme Verlag; 2011

45 Bankhead R, Boullata J, Brantley $S$ et al. Enteral nutrition practice recommendations. JPEN J Parenter Enteral Nutr 2009; 33: 122 - 167

46 Westaby D, Young A, O'Toole P et al. The provision of a percutaneously placed enteral tube feeding service. Gut 2010; 59: 1592 - 1605

47 Zopf Y, Rabe C, Bruckmoser T et al. Percutaneous endoscopic jejunostomy and jejunal extension tube through percutaneous endoscopic gastrostomy: a retrospective analysis of success, complications and outcome. Digestion 2009; 79: $92-97$

48 Teichgraber UK, Streitparth $F$, Cho $\mathrm{CH}$ et al. Percutaneous pushthrough gastrostomy by applying a CT-guided gastropexy. J Vasc Interv Radiol 2011; 22: 1149-1152

49 Bechtold ML, Matteson ML, Choudhary A et al. Early versus delayed feeding after placement of a percutaneous endoscopic gastrostomy: a meta-analysis. Am J Gastroenterol 2008; 103: 2919-2924

50 Barlow $R$, Price $P$, Reid TD et al. Prospective multicentre randomised controlled trial of early enteral nutrition for patients undergoing major upper gastrointestinal surgical resection. Clin Nutr 2011; 30: $560-566$

51 Crosby J, Duerksen DR. A prospective study of tube- and feeding-related complications in patients receiving long-term home enteral nutrition. JPEN J Parenter Enteral Nutr 2007; 31: 274-277

52 Rabeneck L, Wray NP, Petersen NJ. Long-term outcomes of patients receiving percutaneous endoscopic gastrostomy tubes. J Gen Intern Med 1996; 11: $287-293$

53 Hull MA, Rawlings J, Murray FE et al. Audit of outcome of long-term enteral nutrition by percutaneous endoscopic gastrostomy. Lancet 1993; 341: $869-872$

54 Crosby J, Duerksen D. A retrospective survey of tube-related complications in patients receiving long-term home enteral nutrition. Dig Dis Sci 2005; 50: $1712-1717$

55 Rosenberger $L H$, Newhook T, Schirmer B et al. Late accidental dislodgement of a percutaneous endoscopic gastrostomy tube: an underestimated burden on patients and the health care system. Surg Endosc 2011; 25: $3307-3311$

56 Phillips NM, Endacott $R$. Medication administration via enteral tubes: a survey of nurses' practices. J Adv Nurs 2011; 67: 2586-2592

57 Dandeles LM, Lodolce AE. Efficacy of agents to prevent and treat enteral feeding tube clogs. Ann Pharmacother 2011; 45: 676-680

58 Behnken I, Gaschott T, Stein J. Enteral nutrition: drug administration via feeding tube. Z Gastroenterol 2005; 43: 1231 - 1241

59 Williams NT. Medication administration through enteral feeding tubes. Am J Health Syst Pharm 2008; 65: 2347-2357

60 Marcuard SP, Stegall KS. Unclogging feeding tubes with pancreatic enzyme. JPEN J Parenter Enteral Nutr 1990; 14: 198-200

61 Pfaff A. Anwendungshinweise zu Peroralia. München: Nestle Clinical Nutrition; 2001

62 Zopf Y, Konturek P, Nuernberger A et al. Local infection after placement of percutaneous endoscopic gastrostomy tubes: a prospective study evaluating risk factors. Can J Gastroenterol 2008; 22: 987 - 991

63 Hitchings $H$, Best $C$, Steed $I$. Home enteral tube feeding in older people: consideration of the issues. Br J Nurs 2010; 19: 1150-1154

64 Best $C$, Hitchings $H$. Enteral tube feeding - from hospital to home. Br J Nurs 2010; 19: 174, $176-179$

65 Sharma VK, Howden CW. Meta-analysis of randomized, controlled trials of antibiotic prophylaxis before percutaneous endoscopic gastrostomy. Am J Gastroenterol 2000; 95: 3133 -3136

66 Ruesch S, Walder B, Tramer MR. Complications of central venous catheters: internal jugular versus subclavian access - a systematic review. Crit Care Med 2002; 30: 454-460

67 Sznajder JI, Zveibil FR, Bitterman $H$ et al. Central vein catheterization. Failure and complication rates by three percutaneous approaches. Arch Intern Med 1986; 146: 259-261

68 Mermel L. Central venous catheter-related infections and their prevention: is there enough evidence to recommend tunneling for short-term use? Crit Care Med 1998; 26: 1315-1316

69 Richet $H$, Hubert $B$, Nitemberg $G$ et al. Prospective multicenter study of vascular-catheter-related complications and risk factors for positive central-catheter cultures in intensive care unit patients. J Clin Microbiol 1990; 28: 2520-2525

70 Richards B, Chaboyer W, Bladen T et al. Effect of central venous catheter type on infections: a prospective clinical trial. J Hosp Infect 2003; 54: $10-17$

71 Tokars JI, Cookson ST, McArthur MA et al. Prospective evaluation of risk factors for bloodstream infection in patients receiving home infusion therapy. Ann Intern Med 1999; 131: 340 - 347

72 Hilton E, Haslett TM, Borenstein MT et al. Central catheter infections: single- versus triple-lumen catheters. Influence of guide wires on infection rates when used for replacement of catheters. Am J Med 1988; 84: $667-672$ 
73 Pemberton LB, Lyman B, Lander $V$ et al. Sepsis from triple- vs singlelumen catheters during total parenteral nutrition in surgical or critically ill patients. Arch Surg 1986; 121: $591-594$

74 McCarthy MC, Shives JK, Robison RJ et al. Prospective evaluation of single and triple lumen catheters in total parenteral nutrition. JPEN J Parenter Enteral Nutr 1987; 11: $259-262$

75 O'Grady NP, Alexander M, Dellinger EP et al. Guidelines for the prevention of intravascular catheter-related infections. Centers for Disease Control and Prevention. MMWR Recomm Rep 2002; 51: 1 - 29

76 Polderman $K H$, Girbes AR. Central venous catheter use. Part 2: infectious complications. Intensive Care Med 2002; 28: 18-28

77 Raad I, Umphrey J, Khan A et al. The duration of placement as a predictor of peripheral and pulmonary arterial catheter infections. J Hosp Infect 1993; 23: 17-26

78 Krzywda EA, Andris DA, Edmiston CE. Catheter infections: Diagnosis, etiology, treatment and prevention. Nutrition in Clinical Practice 1999; $14: 178$

79 Timsit JF, Sebille V, Farkas JC et al. Effect of subcutaneous tunneling on internal jugular catheter-related sepsis in critically ill patients: a prospective randomized multicenter study. JAMA 1996; 276: 1416 1420

80 Versleijen MW, Huisman-de Waal GJ, Kock MC et al. Arteriovenous fistulae as an alternative to central venous catheters for delivery of long-term home parenteral nutrition. Gastroenterology 2009; 136: $1577-1584$

81 Mühlebach S, Fasolini F. 10 Jahre Ernährungsteam am Kantonsspital Aarau (KSA): Aufgaben, Entwicklungen, Erfahrungen. Viszeralchirurgie 2000 ; 35 : $69-71$

82 Chang L, Tsai JS, Huang SJ et al. Evaluation of infectious complications of the implantable venous access system in a general oncologic population. Am J Infect Control 2003; 31: 34- 39

83 Sitges-Serra A, Linares J, Perez JL et al. A randomized trial on the effect of tubing changes on hub contamination and catheter sepsis during parenteral nutrition. JPEN J Parenter Enteral Nutr 1985; 9: 322 - 325

84 Maki DG, Botticelli JT, LeRoy ML et al. Prospective study of replacing administration sets for intravenous therapy at 48- vs 72-hour intervals. 72 hours is safe and cost-effective. JAMA 1987; 258: 1777-1781

85 Matlow AG, Kitai I, Kirpalani $\mathrm{H}$ et al. A randomized trial of 72- versus 24-hour intravenous tubing set changes in newborns receiving lipid therapy. Infect Control Hosp Epidemiol 1999; 20: 487-493

86 Raad I, Hanna HA, Awad A et al. Optimal frequency of changing intravenous administration sets: is it safe to prolong use beyond 72 hours? Infect Control Hosp Epidemiol 2001; 22: 136-139

87 Attar A, Messing B. Evidence-based prevention of catheter infection during parenteral nutrition. Curr Opin Clin Nutr Metab Care 2001; 4: $211-218$

88 Alonso-Echanove J, Edwards JR, Richards MJ et al. Effect of nurse staffing and antimicrobial-impregnated central venous catheters on the risk for bloodstream infections in intensive care units. Infect Control Hosp Epidemiol 2003; 24: 916-925

89 Abi-Said D, Raad I, Umphrey J et al. Infusion therapy team and dressing changes of central venous catheters. Infect Control Hosp Epidemiol 1999; 20: 101 - 105

90 Maki DG, Ringer M, Alvarado CJ. Prospective randomised trial of povidone-iodine, alcohol, and chlorhexidine for prevention of infection associated with central venous and arterial catheters. Lancet 1991; 338: $339-343$

91 Mimoz O, Villeminey S, Ragot $S$ et al. Chlorhexidine-based antiseptic solution vs alcohol-based povidone-iodine for central venous catheter care. Arch Intern Med 2007; 167: 2066-2072

92 Garland JS, Alex CP, Mueller CD et al. A randomized trial comparing povidone-iodine to a chlorhexidine gluconate-impregnated dressing for prevention of central venous catheter infections in neonates. Pediatrics 2001; 107: 1431-1436

93 Huang EY, Chen C, Abdullah $F$ et al. Strategies for the prevention of central venous catheter infections: an American Pediatric Surgical Association Outcomes and Clinical Trials Committee systematic review. J Pediatr Surg 2011; 46: 2000-2011

94 Hoffmann KK, Weber DJ, Samsa GP et al. Transparent polyurethane film as an intravenous catheter dressing. A meta-analysis of the infection risks. JAMA 1992; 267: 2072-2076

95 Webster J, Gillies D, O'Riordan E et al. Gauze and tape and transparent polyurethane dressings for central venous catheters. Cochrane Database Syst Rev 2011: (11): CD003827 doi: CD003827
96 Mermel LA. Prevention of intravascular catheter-related infections. Ann Intern Med 2000; 132: 391 - 402

97 Maki DG, Stolz SS, Wheeler S et al. A prospective, randomized trial of gauze and two polyurethane dressings for site care of pulmonary artery catheters: implications for catheter management. Crit Care Med 1994; 22: 1729-1737

98 Bijma R, Girbes AR, Kleijer DJ et al. Preventing central venous catheterrelated infection in a surgical intensive-care unit. Infect Control Hosp Epidemiol 1999; 20: 618-620

99 Laura R, Degl'Innocenti M, Mocali M et al. Comparison of two different time interval protocols for central venous catheter dressing in bone marrow transplant patients: results of a randomized, multicenter study. The Italian Nurse Bone Marrow Transplant Group (GITMO). Haematologica 2000; 85: 275-279

100 Madeo M, Martin CR, Turner C et al. A randomized trial comparing Arglaes (a transparent dressing containing silver ions) to Tegaderm (a transparent polyurethane dressing) for dressing peripheral arterial catheters and central vascular catheters. Intensive Crit Care Nurs 1998; $14: 187$ - 191

101 Kommission fur Krankenhaushygiene und Infektionspravention, Robert Koch-Institute. Prävention Gefäßkatheter-assoziierter Infektionen. Bundesgesundheitsblatt - Gesundheitsforschung - Gesundheitsschutz 2002; 45: 907 -924

102 Gosbell I. Central venous catheter related sepsis: epidemiology, pathogenesis, diagnosis, treatment and prevention. Int Care World 1994; $11: 54-59$

103 Bakker H, Bozzetti F, Staun $M$ et al. Home parenteral nutrition in adults: a european multicentre survey in 1997. ESPEN-Home Artificial Nutrition Working Group. Clin Nutr 1999; 18: 135-140

104 Arenas-Marquez H, Anaya-Prado R, Barrera-Zepeda LM et al. Complications of central venous catheters. Curr Opin Clin Nutr Metab Care 2001; 4: $207-210$

105 Bennegard K, Curelaru I, Gustavsson B et al. Material thrombogenicity in central venous catheterization. I. A comparison between uncoated and heparin-coated, long antebrachial, polyethylene catheters. Acta Anaesthesiol Scand 1982; 26: 112-120

106 Mollenholt P, Eriksson I, Andersson T. Thrombogenicity of pulmonaryartery catheters. Intensive Care Med 1987; 13: 57-59

107 Bern MM, Lokich JJ, Wallach SR et al. Very low doses of warfarin can prevent thrombosis in central venous catheters. A randomized prospective trial. Ann Intern Med 1990; 112: 423 - 428

108 Barrett BB, Andersen JW, Anderson KC. Strategies for the avoidance of bacterial contamination of blood components. Transfusion 1993; 33: $228-233$

109 Bozzetti F, Mariani L, Bertinet DB et al. Central venous catheter complications in 447 patients on home parenteral nutrition: an analysis of over 100.000 catheter days. Clin Nutr 2002; 21: 475-485

110 Moreno JM, Shaffer J, Staun $M$ et al. Survey on legislation and funding of home artificial nutrition in different European countries. Clin Nutr 2001; 20: $117-123$

111 Boraks P, Seale J, Price J et al. Prevention of central venous catheter associated thrombosis using minidose warfarin in patients with haematological malignancies. Br J Haematol 1998; 101: 483-486

112 Veerabagu MP, Tuttle-Newhall J, Maliakkal $R$ et al. Warfarin and reduced central venous thrombosis in home total parenteral nutrition patients. Nutrition 1995; 11: $142-144$

113 Klerk CP, Smorenburg SM, Buller HR. Thrombosis prophylaxis in patient populations with a central venous catheter: a systematic review. Arch Intern Med 2003; 163: 1913-1921

114 Verso M, Agnelli G, Bertoglio $S$ et al. Enoxaparin for the prevention of venous thromboembolism associated with central vein catheter: a double-blind, placebo-controlled, randomized study in cancer patients. J Clin Oncol 2005; 23: 4057-4062

115 Couban S, Goodyear M, Burnell $M$ et al. Randomized placebo-controlled study of low-dose warfarin for the prevention of central venous catheter-associated thrombosis in patients with cancer. J Clin Oncol 2005; 23: $4063-4069$

116 Monturo CA, Dickerson RN, Mullen JL. Efficacy of thrombolytic therapy for occlusion of long-term catheters. JPEN J Parenter Enteral Nutr 1990; $14: 312-314$

117 Fraschini G, Jadeja J, Lawson $M$ et al. Local infusion of urokinase for the lysis of thrombosis associated with permanent central venous catheters in cancer patients. J Clin Oncol 1987; 5: 672-678 
118 Gale GB, O'Connor DM, Chu JY et al. Restoring patency of thrombosed catheters with cryopreserved urokinase. JPEN J Parenter Enteral Nutr 1984; 8: $298-299$

119 Pennington $C R$, Pithie $A D$. Ethanol lock in the management of catheter occlusion. JPEN J Parenter Enteral Nutr 1987; 11: 507-508

120 Bader SG, Balke P, Jonkers-Schuitema CF et al. Evaluation of 6 years use of sodium hydroxide solution to clear partially occluded central venous catheters. Clin Nutr 2007; 26: $141-144$

121 Muhlebach $S$. What the practicing physician must know about giving infusions? Praxis (Bern 1994) 2001; 90: 546-548

122 Allwood MC, Kearney MC. Compatibility and stability of additives in parenteral nutrition admixtures. Nutrition 1998; 14: 697-706

123 Shulman RJ, Reed T, Pitre $D$ et al. Use of hydrochloric acid to clear obstructed central venous catheters. JPEN J Parenter Enteral Nutr 1988; 12: $509-510$

124 Duffy LF, Kerzner B, Gebus Vet al. Treatment of central venous catheter occlusions with hydrochloric acid. J Pediatr 1989; 114: 1002 - 1004

125 Goodwin ML. Using sodium bicarbonate to clear a medication precipitate from a central venous catheter. Journal of Vascular Access Nursing 1991; $1: 23$

126 Richards DM, Deeks JJ, Sheldon TA et al. Home parenteral nutrition: a systematic review. Health Technol Assess 1997; 1: i-iii, 1-59

127 Rijnders BJ, Peetermans WE, Verwaest $C$ et al. Watchful waiting versus immediate catheter removal in ICU patients with suspected catheterrelated infection: a randomized trial. Intensive Care Med 2004; 30: $1073-1080$

128 Krzywda EA, Edmiston CE Jr. Central venous catheter infections. Clinical aspects of microbial etiology and pathogenesis. J Infus Nurs 2002; 25: 29-35

129 Forchielli ML, Gura K, Anessi-Pessina E et al. Success rates and cost-effectiveness of antibiotic combinations for initial treatment of centralvenous-line infections during total parenteral nutrition. JPEN J Parenter Enteral Nutr 2000; 24: 119-125

130 Shirotani $N$, Iino T, Numata $\mathrm{K}$ et al. Complications of central venous catheters in patients on home parenteral nutrition: an analysis of 68 patients over 16 years. Surg Today 2006; 36: 420-424

131 Buchman AL, Scolapio J, Fryer J. AGA technical review on short bowel syndrome and intestinal transplantation. Gastroenterology 2003; 124: $1111-1134$

132 Santarpia L, Alfonsi L, Tiseo D et al. Central venous catheter infections and antibiotic therapy during long-term home parenteral nutrition: an 11-year follow-up study. JPEN J Parenter Enteral Nutr 2010; 34: $254-262$

133 Sitges-Serra A, Girvent M. Catheter-related bloodstream infections. World J Surg 1999; 23: 589-595

134 Mermel LA, Allon M, Bouza E et al. Clinical practice guidelines for the diagnosis and management of intravascular catheter-related infection: 2009 Update by the Infectious Diseases Society of America. Clin Infect Dis 2009; 49: 1-45

135 Raad I, Hanna HA, Alakech B et al. Differential time to positivity: a useful method for diagnosing catheter-related bloodstream infections. Ann Intern Med 2004; 140: 18 - 25

136 Krzywda EA, Andris DA, Edmiston CE Jr et al. Treatment of Hickman catheter sepsis using antibiotic lock technique. Infect Control Hosp Epidemiol 1995; 16: 596-598

137 Capdevila JA, Segarra A, Planes AM et al. Successful treatment of haemodialysis catheter-related sepsis without catheter removal. Nephrol Dial Transplant 1993; 8: 231-234

138 Benoit JL, Carandang G, Sitrin $M$ et al. Intraluminal antibiotic treatment of central venous catheter infections in patients receiving parenteral nutrition at home. Clin Infect Dis 1995; 21: 1286 - 1288

139 Messing B, Man F, Colimon R et al. Antibiotic-lock technique is an effective treatment of bacterial catheter-related sepsis during parenteral nutrition. Clin Nutr 1990; 9: 220-225

140 Gaillard JL, Merlino R, Pajot $N$ et al. Conventional and nonconventional modes of vancomycin administration to decontaminate the internal surface of catheters colonized with coagulase-negative staphylococci. JPEN J Parenter Enteral Nutr 1990; 14: 593 - 597

141 Dugdale DC, Ramsey PG. Staphylococcus aureus bacteremia in patients with Hickman catheters. Am J Med 1990; 89: 137-141

142 Schwartz C, Henrickson KJ, Roghmann K et al. Prevention of bacteremia attributed to luminal colonization of tunneled central venous catheters with vancomycin-susceptible organisms. J Clin Oncol 1990; 8: $1591-1597$
143 Bisseling TM, Willems MC, Versleijen MW et al. Taurolidine lock is highly effective in preventing catheter-related bloodstream infections in patients on home parenteral nutrition: a heparin-controlled prospective trial. Clin Nutr 2010; 29: 464-468

144 Guerin K, Wagner J, Rains K et al. Reduction in central line-associated bloodstream infections by implementation of a postinsertion care bundle. Am J Infect Control 2010; 38: 430-433

145 Soifer NE, Borzak S, Edlin BR et al. Prevention of peripheral venous catheter complications with an intravenous therapy team: a randomized controlled trial. Arch Intern Med 1998; 158: 473-477

146 Trautmann M, Bald M, Kramer A et al. Anforderungen an die Hygiene bei Punktionen und Injektionen. Bundesgesundheitsblatt - Gesundheitsforschung - Gesundheitsschutz 2011; 54: 1135-1144

147 Sherertz RJ, Ely EW, Westbrook DM et al. Education of physicians-intraining can decrease the risk for vascular catheter infection. Ann Intern Med 2000; 132: 641-648

148 Eggimann P, Harbarth S, Constantin MN et al. Impact of a prevention strategy targeted at vascular-access care on incidence of infections acquired in intensive care. Lancet 2000; 355: 1864 -1868

149 Safdar N, Maki DG. The pathogenesis of catheter-related bloodstream infection with noncuffed short-term central venous catheters. Intensive Care Med 2004; 30: 62 -67

150 Klek S, Szybinski P, Sierzega $M$ et al. Commercial enteral formulas and nutrition support teams improve the outcome of home enteral tube feeding. JPEN J Parenter Enteral Nutr 2011; 35: 380 - 385

151 Evans S, MacDonald A, Daly A et al. Home enteral tube feeding in patients with inherited metabolic disorders: safety issues. J Hum Nutr Diet 2007; 20: 440-445

152 Jones BJ, Payne S, Silk DB. Indications for pump-assisted enteral feeding. Lancet 1980; 1: 1057-1058

153 Lee JS, Auyeung TW. A comparison of two feeding methods in the alleviation of diarrhoea in older tube-fed patients: a randomised controlled trial. Age Ageing 2003; 32: 388-393

154 Deutsche Gesellschaft für Ernährung, Österreichische Gesellschaft für Ernährung, Schweizerische Gesellschaft für Ernährungsforschung, Schweizerische Vereinigung für Ernährung. Referenzwerte für die Nährstoffzufuhr. 1. Auflage, 4., korrigierter Nachdruck ed. Bonn: 2012

155 Diätverordnung in der Fassung der Bekanntmachung vom 28. April 2005 (BGBl. I S. 1161), die zuletzt durch Artikel 1 der Verordnung vom 1. Oktober 2010 (BGBl. I S. 1306) geändert worden ist

156 Anderson KR, Norris DJ, Godfrey $L B$ et al. Bacterial contamination of tube-feeding formulas. JPEN J Parenter Enteral Nutr 1984; 8: $673-$ 678

157 Levy J, Van Laethem $Y$, Verhaegen $G$ et al. Contaminated enteral nutrition solutions as a cause of nosocomial bloodstream infection: a study using plasmid fingerprinting. JPEN J Parenter Enteral Nutr 1989; $13: 228-234$

158 Jorgensen L, Trautner F, Engquist A. Microbial contamination of tube feeding solutions. Ugeskr Laeger 1990; 152: 1824-1827

159 Patchell CJ, Anderton A, MacDonald A et al. Bacterial contamination of enteral feeds. Arch Dis Child 1994; 70: 327-330

160 Lucia Rocha Carvalho M, Beninga Morais T, Ferraz Amaral D et al. Hazard analysis and critical control point system approach in the evaluation of environmental and procedural sources of contamination of enteral feedings in three hospitals. JPEN J Parenter Enteral Nutr 2000; 24: 296-303

161 Sullivan MM, Sorreda-Esguerra P, Santos EE et al. Bacterial contamination of blenderized whole food and commercial enteral tube feedings in the Philippines. J Hosp Infect 2001; 49: 268-273

162 Löser C, Hrsg. Unter- und Mangelernährung. Klinik-moderne Therapiestrategien-Budgetrelevanz. Stuttgart: Georg Thieme Verlag; 2011

163 Bundesanzeiger Nr. 188, 2005, 01.09.2005

164 Lampe JW, Effertz ME, Larson JL et al. Gastrointestinal effects of modified guar gum and soy polysaccharide as part of an enteral formula diet. JPEN J Parenter Enteral Nutr 1992; 16: 538 - 544

165 Bouin M, Savoye G, Maillot $C$ et al. How do fiber-supplemented formulas affect antroduodenal motility during enteral nutrition? A comparative study between mixed and insoluble fibers. Am J Clin Nutr 2000; 72: 1040-1046

166 Evans S, Daly A, Davies $P$ et al. Fibre content of enteral feeds for the older child. J Hum Nutr Diet 2009; 22: 414-421

167 Beck AM, Holst M, Rasmussen HH. Oral nutritional support of older (65 years+) medical and surgical patients after discharge from hospital: 
systematic review and meta-analysis of randomized controlled trials. Clin Rehabil 2013; 27: 19-27

168 Botella-Carretero JI, Iglesias B, Balsa JA et al. Effects of oral nutritional supplements in normally nourished or mildly undernourished geriatric patients after surgery for hip fracture: a randomized clinical trial. JPEN J Parenter Enteral Nutr 2008; 32: 120 - 128

169 Weimann A, Schütz T, Lipp T et al. Supportiver Einsatz von Trinknahrung in der ambulanten Versorgung von erwachsenen Patienten ein Algorithmus. Aktuel Ernahrungsmed 2012; 37: 282 - 286

170 Empfehlung der Kommission für Krankenhaushygiene und Infektionsprävention beim Robert Koch-Institut (RKI). Infektionsprävention in Heimen. Bundesgesundheitsblatt - Gesundheitsforschung Gesundheitsschutz 2005; 48: 1061 - 1080

171 Didier ME, Fischer S, Maki DG. Total nutrient admixtures appear safer than lipid emulsion alone as regards microbial contamination: growth properties of microbial pathogens at room temperature. JPEN J Parenter Enteral Nutr 1998; 22: 291 - 296

172 Durand-Zaleski I, Delaunay L, Langeron $O$ et al. Infection risk and costeffectiveness of commercial bags or glass bottles for total parenteral nutrition. Infect Control Hosp Epidemiol 1997; 18: 183-188

173 Frei A, Dinkel R, Kemen $M$ et al. A new model for economic studies of therapies exemplified by postoperative parenteral nutrition. Zentralbl Chir 1997; 122: 358 - 365; discussion 366

174 Pichard C, Schwarz G, Frei A et al. Economic investigation of the use of three-compartment total parenteral nutrition bag: prospective randomized unblinded controlled study. Clin Nutr 2000; 19: 245-251

175 Muhlebach S. Practical aspects of multichamber bags for total parenteral nutrition. Curr Opin Clin Nutr Metab Care 2005; 8: 291 - 295

176 Petros WP, Shank WA Jr. A standardized parenteral nutrition solution: prescribing, use, processing, and material cost implications. Hosp Pharm 1986; 21: 648-649, 654-656

177 Kochevar M, Guenter P, Holcombe B et al. ASPEN statement on parenteral nutrition standardization. JPEN J Parenter Enteral Nutr 2007; 31: $441-448$

178 Arends J, Masin M, Hug MJ. Einleitung einer ambulanten parentalen Ernährung (PE): Mehrflaschen- oder All-In-One-Konzepte? Aktuel Ernahrungsmed 2010; 35: 314-315

179 Armstrong CW, Mayhall CG, Miller KB et al. Prospective study of catheter replacement and other risk factors for infection of hyperalimentation catheters. J Infect Dis 1986; 154: 808-816

180 Tomford JW, Hershey CO. The i.v. therapy team: impact on patient care and costs of hospitalization. NITA 1985; 8: 387-389

181 Nehme AE. Nutritional support of the hospitalized patient. The team concept. JAMA 1980; 243: 1906 - 1908

182 Culkin A, Gabe SM, Bjarnason I et al. A double-blind, randomized, controlled crossover trial of glutamine supplementation in home parenteral nutrition. Eur J Clin Nutr 2008; 62: 575-583

$183 \mathrm{Wu} \mathrm{GH}, \mathrm{Wu} \mathrm{ZH}, \mathrm{Wu} \mathrm{ZG}$. Effects of bowel rehabilitation and combined trophic therapy on intestinal adaptation in short bowel patients. World J Gastroenterol 2003; 9: 2601 -2604

184 Li-Ling, Irving $M$. The effectiveness of growth hormone, glutamine and a low-fat diet containing high-carbohydrate on the enhancement of the function of remnant intestine among patients with short bowel syndrome: a review of published trials. Clin Nutr 2001; 20: 199-204

185 Burns DL, Gill BM. Reversal of parenteral nutrition-associated liver disease with a fish oil-based lipid emulsion (Omegaven) in an adult dependent on home parenteral nutrition. JPEN J Parenter Enteral Nutr 2013; 37: 274-280

186 Goulet O, Antebi $H$, Wolf $C$ et al. A new intravenous fat emulsion containing soybean oil, medium-chain triglycerides, olive oil, and fish oil: a single-center, double-blind randomized study on efficacy and safety in pediatric patients receiving home parenteral nutrition. JPEN J Parenter Enteral Nutr 2010; 34: 485-495

187 Lloyd DA, Paynton SE, Bassett $P$ et al. Assessment of long chain n-3 polyunsaturated fatty acid status and clinical outcome in adults receiving home parenteral nutrition. Clin Nutr 2008; 27: 822-831

188 Puder M, Valim C, Meisel JA et al. Parenteral fish oil improves outcomes in patients with parenteral nutrition-associated liver injury. Ann Surg 2009; 250: 395-402

189 Heller AR, Rossel T, Gottschlich B et al. Omega-3 fatty acids improve liver and pancreas function in postoperative cancer patients. Int J Cancer 2004; 111: $611-616$

190 Antebi H, Mansoor O, Ferrier C et al. Liver function and plasma antioxidant status in intensive care unit patients requiring total parente- ral nutrition: comparison of 2 fat emulsions. JPEN J Parenter Enteral Nutr 2004; 28: $142-148$

191 Cavicchi M, Beau P, Crenn P et al. Prevalence of liver disease and contributing factors in patients receiving home parenteral nutrition for permanent intestinal failure. Ann Intern Med 2000; 132: 525 - 532

192 Ekema G, Falchetti D, Boroni $G$ et al. Reversal of severe parenteral nutrition-associated liver disease in an infant with short bowel syndrome using parenteral fish oil (Omega-3 fatty acids). J Pediatr Surg 2008; 43: 1191 - 1195

193 Fallon EM, Le HD, Puder M. Prevention of parenteral nutrition-associated liver disease: role of omega-3 fish oil. Curr Opin Organ Transplant 2010; 15 : 334-340

194 Maroulis J, Kalfarentzos F. Complications of parenteral nutrition at the end of the century. Clin Nutr 2000; 19: 295-304

195 Angelico M, Della Guardia P. Review article: hepatobiliary complications associated with total parenteral nutrition. Aliment Pharmacol Ther 2000; 14 (Suppl. 02): 54-57

196 Chung C, Buchman AL. Postoperative jaundice and total parenteral nutrition-associated hepatic dysfunction. Clin Liver Dis 2002; 6: 1067 1084

197 Wengler A, Micklewright A, Hebuterne X et al. Monitoring of patients on home parenteral nutrition (HPN) in Europe: a questionnaire based study on monitoring practice in 42 centres. Clin Nutr 2006; 25: 693 700

198 Buchman AL. Complications of long-term home total parenteral nutrition: their identification, prevention and treatment. Dig Dis Sci 2001; 46: $1-18$

199 Howard L, Ashley C. Management of complications in patients receiving home parenteral nutrition. Gastroenterology 2003; 124: $1651-1661$

200 Beau P, Labat-Labourdette J, Ingrand P et al. Is ursodeoxycholic acid an effective therapy for total parenteral nutrition-related liver disease? J Hepatol 1994; 20: 240-244

201 Kowdley $K V$. Ursodeoxycholic acid therapy in hepatobiliary disease. Am J Med 2000; 108: 481 - 486

202 Sitzmann JV, Pitt HA, Steinborn PA et al. Cholecystokinin prevents parenteral nutrition induced biliary sludge in humans. Surg Gynecol Obstet 1990; 170: 25-31

203 Doty JE, Pitt HA, Porter-Fink V et al. Cholecystokinin prophylaxis of parenteral nutrition-induced gallbladder disease. Ann Surg 1985; 201: $76-80$

204 Kalfarentzos F, Vagenas C, Michail A et al. Gallbladder contraction after administration of intravenous amino acids and long-chain triacylglycerols in humans. Nutrition 1991; 7: 347 - 349

205 Haderslev KV, Tjellesen L, Haderslev PH et al. Assessment of the longitudinal changes in bone mineral density in patients receiving home parenteral nutrition. JPEN J Parenter Enteral Nutr 2004; 28: 289-294

206 Ferrone M, Geraci M. A review of the relationship between parenteral nutrition and metabolic bone disease. Nutr Clin Pract 2007; 22: 329 339

207 Pironi L, Labate AM, Pertkiewicz M et al. Prevalence of bone disease in patients on home parenteral nutrition. Clin Nutr 2002; 21: 289-296

208 Cohen-Solal M, Baudoin C, Joly F et al. Osteoporosis in patients on long-term home parenteral nutrition: a longitudinal study. J Bone Miner Res 2003; 18: 1989-1994

209 Cauley JA, Black D, Boonen $S$ et al. Once-yearly zoledronic acid and days of disability, bed rest, and back pain: randomized, controlled HORIZON Pivotal Fracture Trial. J Bone Miner Res 2011; 26: 984-992

210 Haderslev KV, Tjellesen L, Sorensen HA et al. Effect of cyclical intravenous clodronate therapy on bone mineral density and markers of bone turnover in patients receiving home parenteral nutrition. Am J Clin Nutr 2002; 76: $482-488$

211 Cummings SR, San Martin J, McClung MR et al. Denosumab for prevention of fractures in postmenopausal women with osteoporosis. N Engl J Med 2009; 361: 756 - 765

212 Smith MR, Egerdie B, Hernandez Toriz $N$ et al. Denosumab in men receiving androgen-deprivation therapy for prostate cancer. $\mathrm{N}$ Engl J Med 2009; 361: 745 - 755

213 Hernandez MV, Peris P, Monegal A et al. Effects of intravenous pamidronate on renal function, bone mineral metabolism and bone mass in patients with severe osteoporosis. Am J Med Sci 2010; 339: 225 229

214 Nishikawa RA, Siepler SE, Siepler JK et al. Intravenous pamidronate improves bone mineral density in home parenteral nutrition patients. Clinical Nutrition 2003; 22 (Suppl. 01): S88 
215 Raman M, Aghdassi E, Baun M et al. Metabolic bone disease in patients receiving home parenteral nutrition: a Canadian study and review. JPEN J Parenter Enteral Nutr 2006; 30: 492 - 496

216 Holick MF. Vitamin D deficiency. N Engl J Med 2007; 357: 266 - 281

217 Acca M, Ragno A, Francucci CM et al. Metabolic bone diseases during long-term total parenteral nutrition. J Endocrinol Invest 2007; 30: 54-59

218 Wanten G, Calder PC, Forbes A. Managing adult patients who need home parenteral nutrition. BMJ 2011; 342: d1447

219 Kommission fur Krankenhaushygiene und Infektionspravention, Robert Koch Institute. Public health requirements in punctures and injections. Bundesgesundheitsblatt Gesundheitsforschung Gesundheitsschutz 2011; 54: 1135 - 1144

220 Kommission für Krankenhaushygiene und Infektionsprävention. Händehygiene. Bundesgesundheitsblatt - Gesundheitsforschung Gesundheitsschutz 2000; 43: 230-233

221 Schmutz C. Microbiological safety and stability of TPN mixtures. XVIII ESPEN Congress on Clinical Nutrition and Metabolism. Geneva: 1996

222 Curtis C, Sacks GS. Compounding parenteral nutrition: reducing the risks. Nutr Clin Pract 2009; 24: $441-446$

223 Bundesverband Deutscher Krankenhausapotheker e.V. (ADKA). ADKA-Leitlinie: Aseptische Herstellung und Prüfung applikationsfertiger Parenteralia. Krankenhauspharmazie 2013; 344: 88 - 106

224 Apothekenbetriebsordnung - ApBetrO in der Fassung der Bekanntmachung vom 26. September 1995 (BGBL. I S. 1195), zuletzt geändert durch Artikel 1 der Vierten Verordnung zur Änderung der Apothekenbetriebsordnung vom 11.06.2012 (BGBL. I S. 1254), hier insbesondere §35: Herstellung von Arzneimitteln zur parenteralen Anwendung [I, legal]

225 Leitlinie der Bundesapothekerkammer zur Qualitätssicherung: Herstellung und Prüfung applikationsfertiger Parenteralia ohne CMREigenschaften der Kategorie 1A oder 1B. Stand der Revision: 08.05.2012. http://www.abda.de/fileadmin/assets/Praktische_Hilfen /Leitlinien/Parenteraliaherstellung/LL_Parenteralia_nichttoxisch.pdf (abgerufen am 26.11. 2012)

226 Steger PJ, Muhlebach SF. Lipid peroxidation of intravenous lipid emulsions and all-in-one admixtures in total parenteral nutrition bags: the influence of trace elements. JPEN J Parenter Enteral Nutr 2000; 24: $37-41$

227 Mühlebach S. Heimparenterale Ernährung: Internationale Entwicklungen und Langzeiterfahrungen aus der Schweiz. Aktuel Ernahrungsmed 2002; 27: 425-430

228 Driscoll DF. Compounding TPN admixtures: then and now. JPEN J Parenter Enteral Nutr 2003; 27: 433 -438; quiz 439

229 Hardy G, Ball P, McElroy B. Basic principles for compounding all-inone parenteral nutrition admixtures. Curr Opin Clin Nutr Metab Care 1998; 1: $291-296$

230 Hartmann B, Berchtold W, Aeberhard P et al. Umfang, Rationalität und Qualität der parenteralen Ernährung. Aktuel Ernahrungsmed 1998; 23: $43-49$

231 Schmutz $C$, Werner $R$, Keller $U$ et al. Emulsion stability of all-in-one TPN admixtures assessed by microscopy. Comparison of different lipid emulsions and amino acid solutions. Clinical Nutrition 1993; 12 : $59-60$

232 Driscoll DF, Bhargava HN, Li L et al. Physicochemical stability of total nutrient admixtures. Am J Health Syst Pharm 1995; 52: $623-634$

233 ASHP guidelines on the safe use of automated compounding devices for the preparation of parenteral nutrition admixtures. Developed through the ASHP Council on Professional Affairs and approved by the ASHP Board of Directors on April 27, 2000. Am J Health Syst Pharm 2000; 57: 1343 - 1348

234 ASHP guidelines on quality assurance for pharmacy-prepared sterile products. American Society of Health System Pharmacists. Am J Health Syst Pharm 2000; 57: 1150-1169

235 McKinnon BT. FDA safety alert: hazards of precipitation associated with parenteral nutrition. Nutr Clin Pract 1996; 11: 59-65

236 Takagi J, Khalidi N, Wolk RA et al. Sterility of total parenteral nutrient solutions stored at room temperature for seven days. Am J Hosp Pharm 1989; 46: 973-977

237 Trissel LA, Gilbert DL, Martinez JF et al. Compatibility of medications with 3-in-1 parenteral nutrition admixtures. JPEN J Parenter Enteral Nutr 1999; 23: $67-74$

238 Barnett MI, Cosslett AG, Duffield JR et al. Parenteral nutrition. Pharmaceutical problems of compatibility and stability. Drug Saf 1990; 5 (Suppl. 01): $101-106$
239 Schmid U, Mühlebach S. Interactions of drugs and i.v. lipids: influence of cyclosporine on fat clearance in vitro. Clinical Nutrition 2011;20: 27

240 Apothekenbetriebsordnung - ApBetrO in der Fassung der Bekanntmachung vom 26. September 1995 (BGBL. I S. 1195), zuletzt geändert durch Artikel 1 der Vierten Verordnung zur Änderung der Apothekenbetriebsordnung vom 11.06.2012 (BGBL. I S. 1254), hier insbesondere $§ 14$ Kennzeichnung von Rezepturarzneimitteln

241 Messing B, Crenn P, Beau $P$ et al. Long-term survival and parenteral nutrition dependence in adult patients with the short bowel syndrome. Gastroenterology 1999; 117: 1043 - 1050

242 Smith CE, Curtas S, Werkowitch $M$ et al. Home parenteral nutrition: does affiliation with a national support and educational organization improve patient outcomes? JPEN J Parenter Enteral Nutr 2002; 26: $159-163$

243 Kovacevich DS, Frederick A, Kelly D et al. Standards for specialized nutrition support: home care patients. Nutr Clin Pract 2005; 20: 579 590

244 Senkal M, Dormann A, Stehle P et al. Survey on structure and performance of nutrition-support teams in Germany. Clin Nutr 2002; 21 : $329-335$

245 Paccagnella A, Mauri A, Berto $R$ et al. Biopsychosocial approach to home enteral nutrition: measure of subjective satisfaction and quality of life. Minerva Med 2007; 98: 5-17

246 Gifford H, Delegge M, Epperson LA. Education methods and techniques for training home parenteral nutrition patients. Nutr Clin Pract 2010; 25: $443-450$

247 Gianino MS, Brunt LM, Eisenberg PG. The impact of a nutritional support team on the cost and management of multilumen central venous catheters. J Intraven Nurs 1992; 15: 327 - 332

248 Goldstein M, Braitman LE, Levine GM. The medical and financial costs associated with termination of a nutrition support nurse. JPEN J Parenter Enteral Nutr 2000; 24: 323 - 327

249 Trujillo EB, Young LS, Chertow GM et al. Metabolic and monetary costs of avoidable parenteral nutrition use. JPEN J Parenter Enteral Nutr 1999; 23: $109-113$

250 Maswoswe JJ, Newcomer DR, Quandt CM. Achieving parenteral nutrition cost savings through prescribing guidelines and formulary restrictions. Am J Hosp Pharm 1987; 44: 1376-1381

251 Roberts MF, Levine GM. Nutrition support team recommendations can reduce hospital costs. Nutr Clin Pract 1992; 7: 227-230

252 Naylor CJ, Griffiths RD, Fernandez RS. Does a multidisciplinary total parenteral nutrition team improve patient outcomes? A systematic review. JPEN J Parenter Enteral Nutr 2004; 28: 251 - 258

253 Agostoni C, Axelson I, Colomb V et al. The need for nutrition support teams in pediatric units: a commentary by the ESPGHAN committee on nutrition. J Pediatr Gastroenterol Nutr 2005; 41: 8-11

254 Howard P, Jonkers-Schuitema C, Furniss L et al. Managing the patient journey through enteral nutritional care. Clin Nutr 2006; 25: 187 195

255 Schneider PJ. Nutrition support teams: an evidence-based practice. Nutr Clin Pract 2006; 21: 62-67

256 Fuhrman MP, Galvin TA, Ireton-Jones CS et al. American Dietetic Association. Practice paper of the American Dietetic Association: Home care - opportunities for food and nutrition professionals. J Am Diet Assoc 2009; 109: $1092-1100$

257 Klein S. A primer of nutritional support for gastroenterologists. Gastroenterology 2002; 122: 1677-1687

258 Hartl WH, Jauch KW, Parhofer $K$ et al. Working group for developing the guidelines for parenteral nutrition of The German Association for Nutritional Medicine. Complications and monitoring - Guidelines on Parenteral Nutrition, Chapter 11. Ger Med Sci 2009; 7: Doc17

259 Ladefoged K. Quality of life in patients on permanent home parenteral nutrition. JPEN J Parenter Enteral Nutr 1981; 5: 132-137

260 Carlson GL, Maguire G, Williams N et al. Quality of life on home parenteral nutrition: a single centre study of 37 patients. Clin Nutr 1995 14: $219-228$

261 Smith CE, Curtas S, Kleinbeck SV et al. Clinical trial of interactive and videotaped educational interventions reduce infection, reactive depression, and rehospitalizations for sepsis in patients on home parenteral nutrition. JPEN J Parenter Enteral Nutr 2003; 27: 137-145

262 Malone $M$. Longitudinal assessment of outcome, health status, and changes in lifestyle associated with long-term home parenteral and enteral nutrition. JPEN J Parenter Enteral Nutr 2002; 26: 164-168 
263 Messing B, Landais P, Goldfarb B et al. Home parenteral nutrition in adults: a multicentre survey in Europe. Clin Nutr 1989; 8: 3-9

264 Jamieson $C P$, Norton B, Day $T$ et al. The quantitative effects of nutrition support on quality of life in outpatients. Clinical Nutrition 1997; 16: $25-28$

265 Stanga Z, Field J, Iff $S$ et al. The effect of nutritional management on the mood of malnourished patients. Clin Nutr 2007; 26: 379-382

266 Howard L. Home parenteral nutrition: survival, cost, and quality of life. Gastroenterology 2006; 130: 52 - 59

267 Baxter JP, Fayers PM, McKinlay AW. The clinical and psychometric validation of a questionnaire to assess the quality of life of adult patients treated with long-term parenteral nutrition. JPEN J Parenter Enteral Nutr 2010; 34: 131 - 142

268 Mueller M, Lohmann S, Thul P et al. Functioning and health in patients with cancer on home-parenteral nutrition: a qualitative study. Health Qual Life Outcomes 2010; 8: 41

269 Gomez Candela C, Cos Blanco A, Garcia Luna PP et al. Complications of enteral nutrition at home. Results of a multicentre trial. Nutr Hosp 2003; 18: $167-173$

270 Silver HJ, Wellman NS, Arnold DJ et al. Older adults receiving home enteral nutrition: enteral regimen, provider involvement, and health care outcomes. JPEN J Parenter Enteral Nutr 2004; 28: 92 -98

271 Huisman-de Waal G, van Achterberg T, Jansen J et al. "High-tech" home care: overview of professional care in patients on home parenteral nutrition and implications for nursing care. J Clin Nurs 2011; 20 : $2125-2134$

272 Bonifacio R, Alfonsi L, Santarpia L et al. Clinical outcome of long-term home parenteral nutrition in non-oncological patients: a report from two specialised centres. Intern Emerg Med 2007; 2: 188 - 195

273 Pironi L, Joly F, Forbes A et al. Long-term follow-up of patients on home parenteral nutrition in Europe: implications for intestinal transplantation. Gut 2011; 60: 17-25

274 Messing B, Joly F. Guidelines for management of home parenteral support in adult chronic intestinal failure patients. Gastroenterology 2006; 130: $43-51$

275 Forchielli ML, Richardson D, Folkman J et al. Better living through chemistry, constant monitoring, and prompt interventions: 26 years on home parenteral nutrition without major complications. Nutrition 2008; 24: $103-107$

276 Kelly DG. Guidelines and available products for parenteral vitamins and trace elements. JPEN J Parenter Enteral Nutr 2002; 26: 34-36

277 van der Hulst RR, von Meyenfeldt MF, Tiebosch A et al. Glutamine and intestinal immune cells in humans. JPEN J Parenter Enteral Nutr 1997; $21: 310-315$

278 Hallum NS, Tan LB, Baxter JP et al. Home parenteral nutrition: Outcome and seven year prospective follow up in a nationwide adult population. e-SPEN Journal 2012; 7: e30-e34

279 Llop J, Sabin P, Garau M et al. The importance of clinical factors in parenteral nutrition-associated hypertriglyceridemia. Clin Nutr 2003; 22: $577-583$

280 Norman JL, Crill CM. Optimizing the transition to home parenteral nutrition in pediatric patients. Nutr Clin Pract 2011; 26: 273-285
281 Evans S, Preston F, Daly A et al. Accuracy of home enteral feed preparation for children with inherited metabolic disorders. J Hum Nutr Diet 2011; 24: 68-73

282 Evans S, Shelton F, Holden C et al. Monitoring of home safety issues in children on enteral feeds with inherited metabolic disorders. Arch Dis Child 2010; 95: 668-672

283 Silver HJ, Wellman NS, Galindo-Ciocon D et al. Family caregivers of older adults on home enteral nutrition have multiple unmet taskrelated training needs and low overall preparedness for caregiving. J Am Diet Assoc 2004; 104: $43-50$

284 Kaiser MJ, Bauer JM, Ramsch $C$ et al. Frequency of malnutrition in older adults: a multinational perspective using the mini nutritional assessment. J Am Geriatr Soc 2010; 58: 1734-1738

285 Milne AC, Avenell A, Potter J. Meta-analysis: protein and energy supplementation in older people. Ann Intern Med 2006; 144: 37-48

286 Deutsches Netzwerk für Qualitätsentwicklung in der Pflege (DNQP). Expertenstandard Ernährungsmanagement zur Sicherstellung und Förderung der oralen Ernährung in der Pflege. Osnabrück: 2010

287 Aangenendt-Siegers IP, Cools HJ. Cause of death in nursing homes. Ned Tijdschr Geneeskd 1992; 136: 2015-2017

288 Day HB. The treatment of infantile diarrhea by saline injections. The Practitioner 1993; 91: 58-64

289 O'Keeffe ST, Lavan JN. Subcutaneous fluids in elderly hospital patients with cognitive impairment. Gerontology 1996; 42: 36-39

290 Arinzon Z, Feldman J, Fidelman Z et al. Hypodermoclysis (subcutaneous infusion) effective mode of treatment of dehydration in longterm care patients. Arch Gerontol Geriatr 2004; 38: 167-173

291 Slesak G, Schnurle JW, Kinzel E et al. Comparison of subcutaneous and intravenous rehydration in geriatric patients: a randomized trial. J Am Geriatr Soc 2003; 51: 155-160

292 Constans T, Dutertre JP, Froge E. Hypodermoclysis in dehydrated elderly patients: local effects with and without hyaluronidase. J Palliat Care 1991; 7: 10-12

293 Hypodermoclysis Working Group. Hypodermoclysis Guidelines on the technique. 1998

294 Ferry M, Leverve X, Constans T. Comparison of subcutaneous and intravenous administration of a solution of amino acids in older patients. J Am Geriatr Soc 1997; 45: 857-860

295 Bruera E, de Stoutz ND, Fainsinger RL et al. Comparison of two different concentrations of hyaluronidase in patients receiving one-hour infusions of hypodermoclysis. J Pain Symptom Manage 1995; 10: 505-509

296 Lipschitz S, Campbell AJ, Roberts MS et al. Subcutaneous fluid administration in elderly subjects: validation of an under-used technique. J Am Geriatr Soc 1991; 39: 6-9

297 Lybarger $E H$. Hypodermoclysis in the home and long-term care settings. J Infus Nurs 2009; 32: 40-44

298 Lee JS, Kwok T, Chui PY et al. Can continuous pump feeding reduce the incidence of pneumonia in nasogastric tube-fed patients? A randomized controlled trial. Clin Nutr 2010; 29: 453-458 